NISTIR 8342

\title{
A Comparison of Measurement Methods for Alcohol-Based Hand Sanitizers
}

\author{
Mary Bedner \\ Jacolin A. Murray \\ Aaron A. Urbas \\ William A. MacCrehan \\ Walter B. Wilson
}

This publication is available free of charge from:

https://doi.org/10.6028/NIST.IR.8342

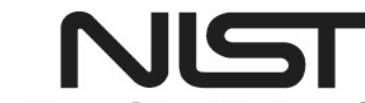

National Institute of Standards and Technology U.S. Department of Commerce 
NISTIR 8342

\title{
A Comparison of Measurement Methods for Alcohol-Based Hand Sanitizers
}

\author{
Mary Bedner \\ Jacolin A. Murray \\ Aaron A. Urbas \\ William A. MacCrehan \\ Walter B. Wilson \\ Chemical Sciences Division \\ Material Measurement Laboratory
}

This publication is available free of charge from:

https://doi.org/10.6028/NIST.IR.8342

February 2021

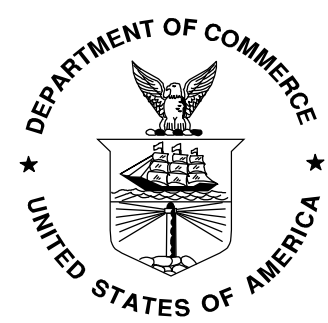

U.S. Department of Commerce

Wynn Coggins, Acting Secretary

National Institute of Standards and Technology James K. Olthoff, Performing the Non-Exclusive Functions and Duties of the Under Secretary of Commerce for Standards and Technology \& Director, National Institute of Standards and Technology 
Certain commercial entities, equipment, or materials may be identified in this document in order to describe an experimental procedure or concept adequately. Such identification is not intended to imply recommendation or endorsement by the National Institute of Standards and Technology, nor is it intended to imply that the entities, materials, or equipment are necessarily the best available for the purpose.

National Institute of Standards and Technology Interagency or Internal Report 8342 Natl. Inst. Stand. Technol. Interag. Intern. Rep. 8342, 63 pages (February 2021)

This publication is available free of charge from: https://doi.org/10.6028/NIST.IR.8342 


\begin{abstract}
The production and demand for hand sanitizers have increased dramatically during the coronavirus disease 2019 (COVID-19) health emergency. To be deemed effective and safe, hand sanitizers should contain at least $60 \%$ alcohol (typically ethanol) as well as minimal amounts of harmful impurities regulated by the Food and Drug Administration (FDA). To help ensure product potency and safety through sound measurements, the National Institute of Standards and Technology (NIST) developed and evaluated four instrumental measurement approaches for their applicability in measuring ethanol and impurities in 72 hand sanitizers representing a range of brands and formulations. The methods included gas chromatography with flame ionization detection (GC-FID), liquid chromatography with ultraviolet absorbance detection (LC-UV), quantitative nuclear magnetic resonance spectroscopy (qNMR), and attenuated total reflectance Fourier-transform infrared spectroscopy (ATR-FTIR). All four instrumental methods can determine and provide comparable results for ethanol, the principle disinfectant in different hand sanitizer formulations. All methods can also confirm the presence of other alcohols potentially present in significant quantities ( $\approx$ percent levels) such as methanol, a harmful impurity, and isopropanol, which can be either the primary disinfectant or an approved denaturant in some formulations. Two of the methods, qNMR and GC-FID, were also able to determine impurities at the requisite sensitivity levels $(\mu \mathrm{g} / \mathrm{g})$ set by the FDA limits. This report presents descriptions and key results from each method. In addition, a discussion regarding the applicability and strengths and weaknesses of each measurement approach for the analysis of hand sanitizers is presented and discussed.
\end{abstract}

\title{
Key words
}

Hand sanitizer; Ethanol; Gas chromatography; Flame ionization detection; Nuclear magnetic resonance spectroscopy; Fourier-transform infrared spectroscopy; Liquid chromatography; Impurities. 
Table of Contents

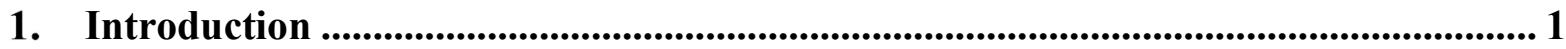

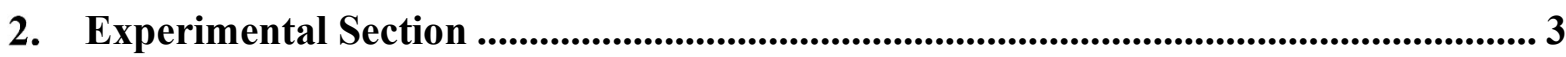

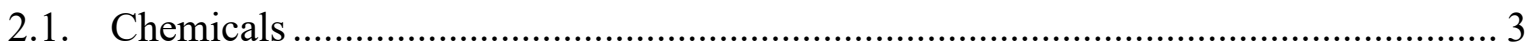

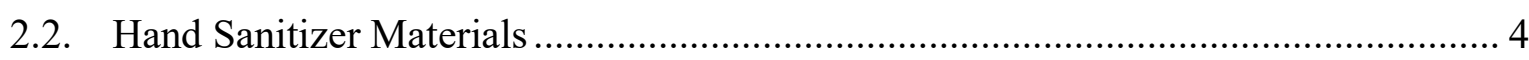

2.3. Mass-Volume Considerations for Ethanol Mixtures................................................. 4

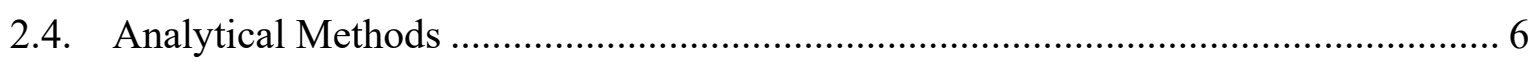

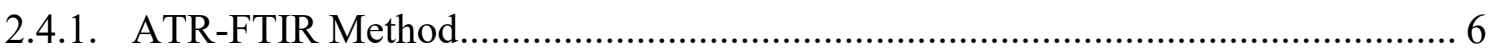

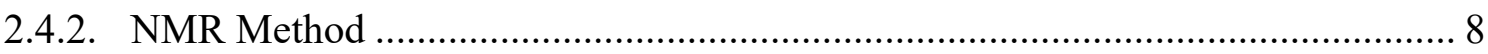

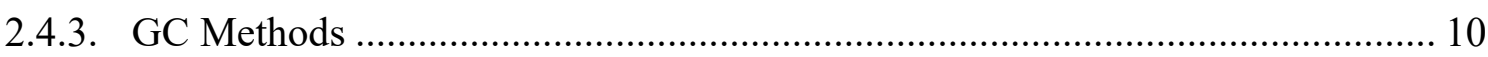

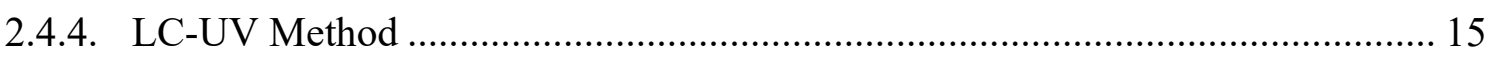

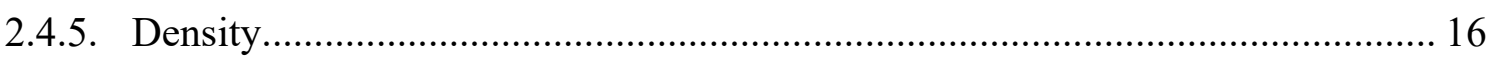

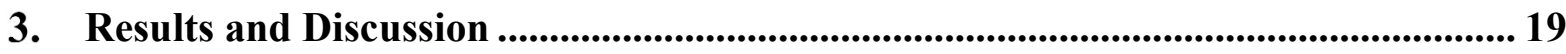

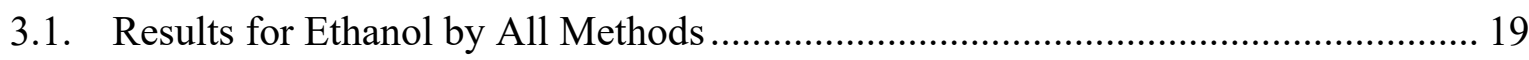

3.2. Results for Methanol and Isopropanol by Multiple Methods.................................... 23

3.3. Individual Methods Results and Discussion ............................................................ 25

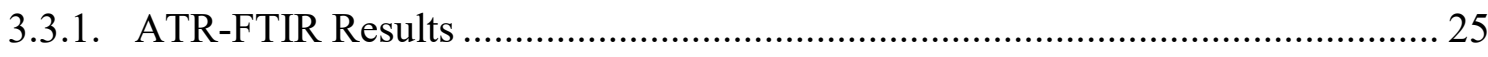

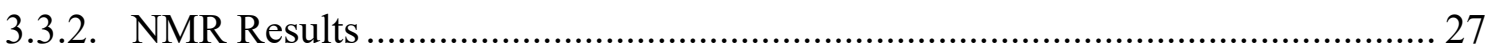

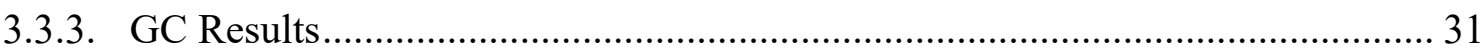

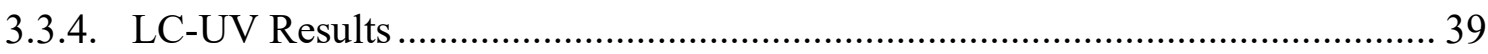

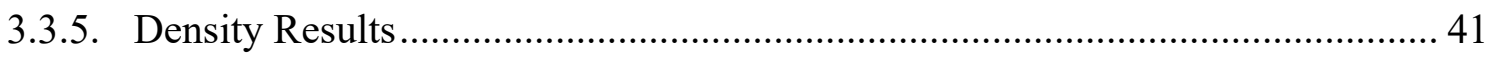

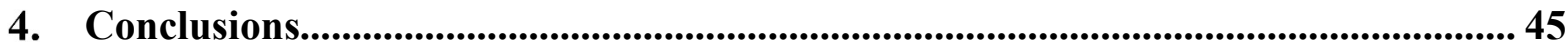

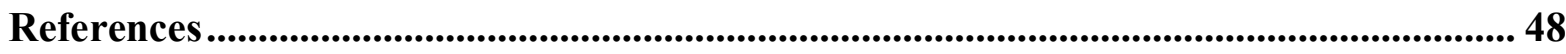

Appendix A: A summary of label information and product consistency for hand sanitizers analyzed. ..................................................................................................................... 49

Appendix B: NMR estimated results for impurities below FDA interim limits. ..............53

Appendix C. Plots of GC-FID results for impurities below FDA limits in some hand

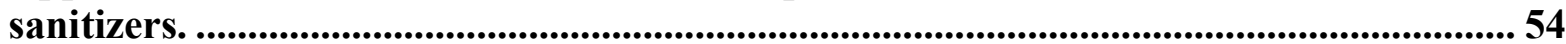

Appendix D. Density results for hand sanitizers $(\mathrm{g} / \mathrm{mL})$ obtained using a calibrated positive displacement pipette. ............................................................................................................... 55

\section{List of Tables}

Table 1. FDA Interim Limits for Impurities in Hand Sanitizers (2020) [5-7] ........................ 2

Table 2. Chemical Reagents for Hand Sanitizer Ingredients and Impurities........................... 3 
Table 3. Preparation of stock and intermediate stock solutions for impurity compound

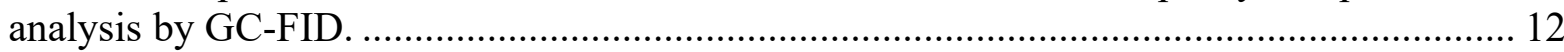

Table 4. Calibration curve information for the impurities quantified by GC-FID............... 14

Table 5. Results for ethanol (mass \%) in 18 hand sanitizers by four methods. .................... 21

Table 6. Results for ethanol (volume \%) in 18 hand sanitizers by four methods along with the average method result for comparison to the CDC minimum value and the product label.

Table 7. Summary of results for methanol (mass \%) in 5 hand sanitizers by three methods. 24 Table 8. Summary of results for isopropanol (volume \%) in 6 hand sanitizers by three methods.

Table 9. Quantitative GC-FID results for impurities over the FDA limits in select hand sanitizers.

Table 10. Quantitative GC-FID results for impurities below the FDA limits in select hand sanitizers.

Table 11. Density results for select hand sanitizers obtained using three techniques........... 42

Table 12. Comparing the ethanol volume \% predicted from density to measured values by ATR-FTIR for hand sanitizers believed to follow the WHO/FDA guidance.

Table 13. Summary of method suitability for hand sanitizer measurements.

\section{List of Figures}

Figure 1. Relationship of volume $\%$ and mass $\%$ for ethanol in water mixtures at $20{ }^{\circ} \mathrm{C}$...... 5

Figure 2. Spectra of calibrants used for the ATR-FTIR method. ..................................... 8

Figure 3. Calibration plots of LC chromatographic peak area versus alcohol \%. ............... 16

Figure 4. Determination of density of hand sanitizers with a hydrometer.......................... 18

Figure 5. Plots of NIST method results for ethanol (volume \%) in select hand sanitizers.... 22

Figure 6. Comparison of the NIST average result to the product label claim for ethanol

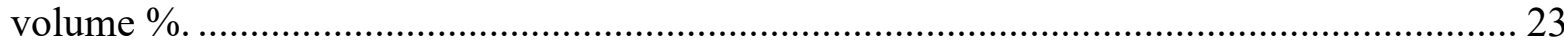

Figure 7. ATR-FTIR spectra of hand sanitizers compared to ethanol and methanol. .......... 26

Figure 8. Estimated ethanol volume \% in hand sanitizers by ATR-FTIR ......................... 27

Figure 9. Example NMR spectrum of a hand sanitizer (Sample 2) showing ethanol, other

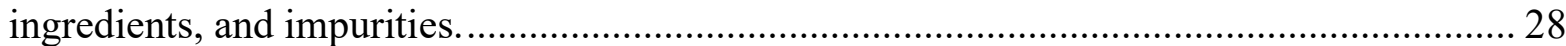

Figure 10. NMR estimates of denaturants in hand sanitizers. ........................................ 29

Figure 11. NMR estimates of impurities above the FDA limits in some hand sanitizers...... 30

Figure 12. Example GC-FID chromatograms of simple, non-gel formulation hand sanitizers.

Figure 13. Example GC-FID chromatograms for more complex (gel) hand sanitizer formulations and a calibrant.....

Figure 14. Example GC-FID chromatograms for impurities in two hand sanitizers and a calibrant. 33

Figure 15. Plots of GC-FID results for impurities above FDA limits in some hand sanitizers.

Figure 16. Separation and detection of a non-gel hand sanitizer (upper chromatogram) and an alcohol mixture (lower chromatogram) using LC-UV. 40

Figure 17. Plot of predicted (density) versus measured (ATR-FTIR) ethanol volume \% results for hand sanitizers following the WHO/FDA formula. 
Figure 18. Plot of predicted (density) versus measured (ATR-FTIR) ethanol volume \% results for all hand sanitizers in this study.

Figure 19. Summary of results for ethanol and impurities in most hand sanitizers evaluated in this work.

\section{ACRONYMS AND SYMBOLS}

ACS grade reagent meets or exceeds purity standards of the American Chemical Society

ATR attenuated total reflectance

$\mathrm{C}_{18}$ octyldecylsilane, analytical column for LC

$\mathrm{CDC}$ Centers for Disease Control and Prevention

COVID-19 coronavirus disease 2019, officially known as SARS-CoV-2

$\mathrm{D}_{2} \mathrm{O}$ deuterium oxide

DMSO

dimethyl sulfoxide

$\mathrm{DMSO}_{2}$ dimethyl sulfone

FID flame ionization detection

FDA Food and Drug Administration

FTIR

GC Fourier transform infrared spectroscopy

gel

$\mathrm{HCl}$ gas chromatography

HPLC-grade

ID

KHP a liquid hand sanitizer with medium or high viscosity hydrochloric acid

$\mathrm{LC}$

LOD a solvent of quality for use in high performance liquid chromatography identifier code used for hand sanitizers

mass $\%$

MS potassium hydrogen phthalate

$m / z$ liquid chromatography limit of detection mass fraction expressed in percent mass spectrometry mass-to-charge ratio (for MS)

NIST

NISTIR

NMR

National Institute of Standards and Technology

non-gel NIST Internal Report

ppm nuclear magnetic resonance spectroscopy

$\%$ RSD a liquid hand sanitizer with low viscosity mass fraction expressed as parts per million $(\mu \mathrm{g} / \mathrm{g})$

qNMR

$\delta(\mathrm{ppm})$

$\mathrm{RF}$

$\mathrm{SD}$

SRM

USA percent relative standard deviation quantitative nuclear magnetic resonance spectroscopy

USP

UV relative chemical shift, in parts per million of the magnetic field (for NMR) response factor (for chromatography)

standard deviation

volume $\%$ Standard Reference Material United States of America United States Pharmacopeia WHO ultraviolet absorbance volume fraction expressed in percent World Health Organization 


\section{Introduction}

In January 2020, the World Health Organization (WHO) declared the coronavirus disease 2019 (COVID-19) a global pandemic and public health emergency [1]. To help control the spread and decrease the risk of getting sick from this infectious virus [2], the WHO and the United States (U.S.) Centers for Disease Control and Prevention (CDC) recommend proper hand hygiene, including frequent handwashing and the use of alcohol-based hand sanitizers. To be deemed generally effective against germs, the CDC recommends that hand sanitizers should contain at least $60 \%$ alcohol [3], which can be either ethanol (ethyl alcohol) or isopropanol (2-propanol). As a result, the demand and market for hand sanitizer products has increased dramatically in the United States and across the globe.

Hand sanitizers are classified as drugs and are regulated for safety and efficacy by the Food and Drug Administration (FDA) in the USA. Typically, hand sanitizer manufacturers are registered with the FDA and formulate their products using standard ingredients and testing procedures verified by the United States Pharmacopeia (USP). To respond to the increased demand and to ensure that hand sanitizers are widely available to the American public, the FDA has modified its procedures during the public health emergency and has enabled the following guidance and flexibilities for industry [4]: non-traditional manufacturers that are not currently registered to make drugs can register as over-the-counter manufacturers of alcohol-based hand sanitizers [5]; pharmacies and registered outsourcing facilities can compound certain alcohol-based hand sanitizers [6]; and alcohol manufacturers can produce alcohol for use in hand sanitizer if they follow guidance developed for industry [7]. The USP has also recently assembled their standards into a single collection to support hand sanitizer manufacturers during this COVID-19 crisis [8].

These temporary policy changes by the FDA have greatly increased the supply of alcoholbased hand sanitizers on the market. However, this rise in the number of products from nontraditional manufacturers has also correlated with an increase in the number of products with identified safety and efficacy issues. Notably, several hand sanitizers have been recalled by the FDA for containing harmful amounts of impurities including methanol, $n$-propanol, and microbes [9]. There have also been instances where products have been found to be subpotent, containing less than the recommended amount of $60 \%$ ethanol (by volume). To help industry measure the levels of impurities and ethanol in their finished products, the FDA has also recently provided a sophisticated analytical method based on gas chromatography mass spectrometry [10] on their website.

The FDA guidance provides limits for chemical impurities in raw alcohol materials [5-7], which have been interchangeably used as the allowable limits in finished hand sanitizer products [10]. The FDA limits are expressed in parts per million (ppm) and are summarized in Table 1 . We have interpreted ppm to be mass fraction units of $\mu \mathrm{g} / \mathrm{g}$ based on the recent FDA method [10], although it should be noted that the USP collection of monographs and standards provides limits in pure alcohol in units of $\mu \mathrm{L} / \mathrm{L}[8]$.

In response to the COVID-19 health emergency, the National Institute of Standards and Technology (NIST) has sought to support industry through the development and evaluation of four quantitative methods for the analysis of finished hand sanitizer products. The methods 
developed and evaluated by NIST include gas chromatography with flame ionization detection (GC-FID), liquid chromatography with ultraviolet absorbance detection (LC-UV), quantitative nuclear magnetic resonance spectroscopy (qNMR), and attenuated total reflectance Fourier-transform infrared spectroscopy (ATR-FTIR).

Table 1. FDA Interim Limits for Impurities in Hand Sanitizers (2020) [5-7].

\begin{tabular}{|lcc|}
\hline $\begin{array}{c}\text { Hand Sanitizer } \\
\text { Impurity }\end{array}$ & $\begin{array}{c}\text { FDA Interim Limit } \\
\mathbf{p p m}(\boldsymbol{\mu g} / \mathbf{g})\end{array}$ & $\begin{array}{c}\text { FDA Interim Limit } \\
\text { mass } \%\end{array}$ \\
\hline acetal & 50 & 0.005 \\
acetaldehyde & 50 & 0.005 \\
acetone & 4400 & 0.44 \\
amyl alcohol & 4100 & 0.41 \\
benzene & 2 & 0.0002 \\
ethyl acetate & 2200 & 0.22 \\
isoamyl alcohol & 4100 & 0.41 \\
isobutanol & 21700 & 2.17 \\
methanol & 630 & 0.063 \\
$n$-butanol & 1000 & 0.1 \\
$n$-propanol & 1000 & 0.1 \\
sec-butanol & 6200 & 0.62 \\
ppm = parts per million & &
\end{tabular}

The four methods used by NIST have been tailored toward the FDA guidance for industry, primarily to support non-traditional manufacturers that are making hand sanitizers for the first time. The methods have been used to evaluate 'simple' hand sanitizer formulas based on the FDA guidance [5,6] which is in alignment with WHO recommendations [11]. These formulations primarily contain the following ingredients listed in volume percent: $80 \%$ denatured ethanol or $75 \%$ isopropanol, $1.45 \%$ glycerin, $0.125 \%$ hydrogen peroxide, and water. Also, some of the methods were evaluated for more complex formulas containing gelling-agents that more closely resemble 'typical' hand sanitizers found in the marketplace. The NIST methods can determine and provide comparable results for ethanol, which was the principle disinfectant alcohol in all but one of the different hand sanitizer formulations evaluated in this work. All methods can also confirm the presence of other alcohols potentially present in significant quantities ( $\approx$ percent levels) such as isopropanol or the harmful impurity, methanol. Two of the methods, qNMR and GC-FID, were also able to determine other ingredients and impurities at the requisite sensitivity ppm levels set by the FDA limits.

This NIST Internal Report (NISTIR) provides a description of each method, summary of key results, and a discussion of the strengths and weaknesses and applicability of each method for analyzing hand sanitizers. 


\section{Experimental Section}

\subsection{Chemicals}

For all methods except for qNMR, the ethanol measurements in the hand sanitizers were calibrated using either (1) solutions prepared from neat ethanol of known purity determined at NIST, or (2) NIST Standard Reference Material (SRM) 2899a Ethanol-Water Solution (Nominal Mass Fraction $25 \%$ ). The qNMR method was calibrated with NIST

SRM 841 Potassium Hydrogen Phthalate (KHP) or dimethyl sulfone $\left(\mathrm{DMSO}_{2}\right)$ as an internal standard.

Hand sanitizers were also all evaluated for isopropanol and tert-butanol (approved denaturants) and the potential impurities regulated by the FDA as listed in Table 1. Calibrants for the ATR-FTIR method were prepared from ethanol and the reagents water, glycerin, and hydrogen peroxide to mimic WHO/FDA recommended hand sanitizer formulations. Descriptions of the chemical reagents used for this work are provided in Table 2. The chromatographic purities of the standards for the hand sanitizer impurity compounds were estimated using GC-FID at NIST and are also reported in Table 2. Other method-specific solvents and chemicals used for this work are included in the individual method summaries in Section 2.4.

Table 2. Chemical Reagents for Hand Sanitizer Ingredients and Impurities.

\begin{tabular}{|llc|}
\hline Compound/Reagent & $\begin{array}{l}\text { Chemical Standard Grade or Purity } \\
\text { Information from Product Label }\end{array}$ & $\begin{array}{l}\text { NIST Purity Estimated } \\
\text { by GC-FID (\%) }\end{array}$ \\
\hline acetal & $99 \%$ purity & 98.4 \\
acetaldehyde & Puriss, $\geq 99.5 \%$ purity & 92.7 \\
acetone & ACS/HPLC grade & 100 \\
amyl alcohol & 99\% purity & 99.0 \\
benzene & Pesticide grade & 99.9 \\
ethyl acetate & HPLC grade & 99.9 \\
glycerin & USP grade & ND \\
hydrogen peroxide & 3\%, USP grade & ND \\
isoamyl alcohol & ACS grade & 99.7 \\
isobutanol & HPLC grade & 99.9 \\
isopropanol & USP grade & 100 \\
methanol & Purge and Trap grade & 100 \\
$n$-butanol & $99.9 \%$ purity & 99.5 \\
$n$-propanol & HPLC grade; $\geq 99 \%$ purity & 100 \\
sec-butanol & $98 \%$ purity & 99.1 \\
tert-butanol & ACS grade & ND \\
water & HPLC grade & ND \\
\hline
\end{tabular}

$\mathrm{ND}=$ not determined 


\subsection{Hand Sanitizer Materials}

Hand sanitizers for this study were obtained primarily from commercial sources (both online and physical retail stores) over the period from April to July 2020. Exceptions included three materials that were provided to NIST by a local police department and two samples from hand sanitizer dispenser stations (one at NIST and one at a local store). A complete summary of the hand sanitizer materials analyzed is provided in Appendix A. Each hand sanitizer was given a unique sample identifier code (Sample ID) as it is NIST's policy to not identify or potentially discriminate against any possible vendor. For the code, the first number indicates a unique brand, and the subsequent letter represents a different lot of the same brand, where applicable. For one of the materials (brand 39), NIST acquired 12 bottles of the same brand and same lot (lot A), and each material was labeled as 39-A-1 through 39-A-12 to represent the different units. In total, 72 samples across 48 brands were analyzed as part of this study. For each hand sanitizer material, the label active ingredient was noted (typically ethyl alcohol or alcohol) along with the place of manufacture (if listed) and the first six inactive ingredients. Hand sanitizers were further visually characterized by NIST for their consistency - either non-gel (non-viscous) or gel. The consistency of the product impacted the sample preparation for the GC and LC methods, as gels tend to contain carbomer or other polymers that require strong acid to break the crosslinking to prevent the materials from fouling the instrument injection ports or columns. The information about the sample consistency is also provided in Appendix A. Fifty-eight of the sanitizers evaluated were classified by NIST as having a gel consistency and contained multiple inactive ingredients. The remaining 14 were characterized as non-gels and had simpler formulations, most of which were more consistent with the WHO/FDA recommendations.

All hand sanitizers were screened using the ATR-FTIR method to provide a quick assessment of the ethanol content. Additionally, most of the hand sanitizers were analyzed using a qualitative NMR approach to screen for impurity compounds. Of the 72 samples, 31 hand sanitizers (nearly half) were also selected for additional quantitative analysis primarily based on the following four criteria: (1) samples were obtained for analysis from a local (Maryland) police department; (2) samples appeared to have less than the recommended $60 \%$ ethanol (by volume) based on ATR-FTIR results; (3) samples appeared to have detectable amounts of the regulated impurities above the FDA limits based on NMR results; and/or (4) samples appeared to follow the simple WHO/FDA manufacturing formula. The hand sanitizers selected for detailed quantitative analysis based on these criteria are highlighted in bold font in Appendix A.

\subsection{Mass-Volume Considerations for Ethanol Mixtures}

According to the CDC guidelines, hand sanitizers should contain a minimum of $60 \%$ alcohol by volume to have the proper potency for disinfection [3]. While the alcohol can be either ethanol or isopropanol, most hand sanitizers currently in the marketplace contain ethanol as the primary disinfectant, as partially evidenced by the hand sanitizers randomly purchased for this work (Appendix A). Of these samples, all but one hand sanitizer (Sample 44) contained ethanol as the primary disinfectant. Our NIST methods were therefore focused primarily on the reliable quantitation of ethanol in finished hand sanitizer products. 
Ethanol content in hand sanitizers is generally reported in units of volume fraction or mass fraction in percent, which are used throughout this report and are defined as follows:

$$
\begin{gathered}
\text { volume percent }(\%), \text { ethanol }=\frac{\text { volume of ethanol }}{\text { total volume of solution }} \times 100 \\
\text { mass percent }(\%), \text { ethanol }=\frac{\text { mass of ethanol }}{\text { total mass of solution }} \times 100
\end{gathered}
$$

For solutions of ethanol in water, volume \% and mass \% are not equal to each other, and this reality is important to the reporting and interpretation of our measurement results. For ethanol and water solutions like hand sanitizers, the masses of each component combined to prepare the solution are additive and are linear with mass \%. However, solutions made by mixing volumes are not additive and hence are non-linear in volume $\%$. Thus, the relationship between mass $\%$ and volume $\%$ is also non-linear [12], which is graphically presented for simple ethanol-water mixtures as the black curve in Figure 1. Figure 1 also has a red dashed line for visualization purposes that corresponds to a hypothetical linear mixing relationship. It should be noted that the volume $\%$ for ethanol is always larger than the mass $\%$ except for $0 \%$ and $100 \%$, corresponding to pure water and ethanol, respectively. An additional consideration for ethanol determinations is that volume $\%$ is temperature dependent, whereas mass \% is not. However, this is not a major concern when working under controlled laboratory conditions. Using density as a proxy to represent volume changes with temperature, the density of a $60 \%$ ethanol in water solution (by volume) differs by only $0.35 \%$ over the range from $19^{\circ} \mathrm{C}$ to $23{ }^{\circ} \mathrm{C}[13]$.

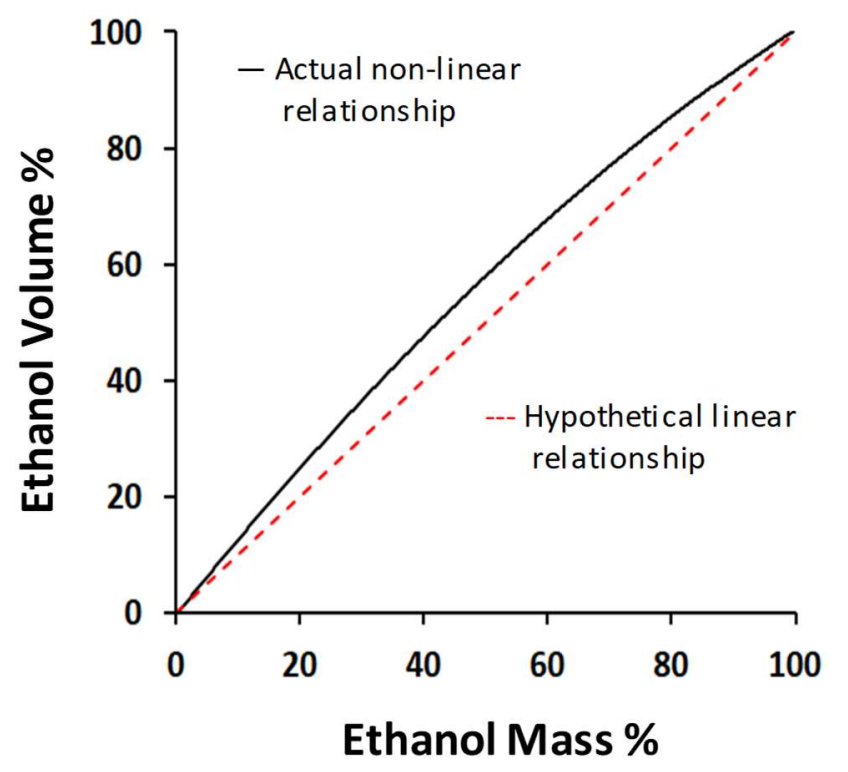

Figure 1. Relationship of volume $\%$ and mass $\%$ for ethanol in water mixtures at $20^{\circ} \mathrm{C}$. 


\subsection{Analytical Methods}

Four different instrumental methods NMR, ATR-FTIR, GC-FID, and LC-UV were evaluated for their ability to determine the content of ethanol, denaturants, and impurities in hand sanitizers.

For the NIST GC-FID and NMR methods, quantitation of ethanol and other components was performed using an internal standard approach with calibrants and samples prepared by mass \%. The use of an internal standard effectively corrects for volume effects and provides the greatest quantitative accuracy when combined with mass measurements, which are not subject to temperature effects or non-additive mixing behavior. Two of the methods, ATRFTIR and LC-UV, utilize spectroscopic detectors that follow Beer's law and tend to be linear with ethanol content in volume $\%$. Therefore, these two methods were calibrated with known solutions of ethanol prepared by mass but converted to volume $\%$ as described in the subsequent sections.

To compare the results for ethanol determined in hand sanitizers using the four different analytical methods, it was necessary to convert between ethanol content in mass \% and in volume \%, which are not equal to each other as described in Section 2.3. These conversions were achieved for the hand sanitizers using the density of pure ethanol and the densities of the individual samples, which were determined by NIST.

Descriptions of the NIST instrumental methods and the approaches used to the estimate the density of the hand sanitizers are provided in the following subsections.

\subsubsection{ATR-FTIR Method}

FTIR offers a rapid analytical approach for estimating the major components in hand sanitizers and, when coupled with ATR, requires no sample preparation. Of specific interest for hand sanitizers is verifying that they contain the recommended minimum amount of alcohol to be effective. ATR-FTIR was utilized to screen every hand sanitizer acquired in this study to estimate ethanol content. A separate calibration was not developed for isopropanol as there was only a single hand sanitizer (Sample 44) obtained with this as the primary active ingredient.

\section{Instrumental}

A Vertex-70 FTIR spectrometer (Bruker Optics) equipped with a DuraScope (Smith's Detection) ATR accessory with a single-bounce diamond element was used to analyze hand sanitizer samples. For this application the standard globar mid-IR source, KBr beam-splitter, and room temperature DLaTGS detector were used. Spectra were collected over the wavenumber range from $550 \mathrm{~cm}^{-1}$ to $4000 \mathrm{~cm}^{-1}$ at $4 \mathrm{~cm}^{-1}$ scanning resolution. Double-sided interferograms were acquired with 32 coadded scans for a total acquisition time of approximately $30 \mathrm{~s}$. Interferograms were processed using a Blackman-Harris 3-term apodization function and $4 \mathrm{X}$ zero-filling followed by Fourier transformation. A new reference spectrum was typically acquired between each sample after cleaning the sample platform. The reference spectrum for all samples was room air (bare crystal). The temperature of the samples, instrument or ATR sample platform were not controlled other than by the lab air handling system. Lab temperature was monitored periodically during data 
collection and found to be consistently between $21^{\circ} \mathrm{C}$ and $22^{\circ} \mathrm{C}$. All samples were equilibrated to lab ambient temperature in bulk before analysis.

To minimize potential impacts from evaporation, flat bottom aluminum sample cups with $5 \mathrm{~mm}$ round openings in the base were placed on the ATR stage to serve as sample reservoirs with a capacity of approximately $0.5 \mathrm{~mL}$. While some viscous gel formulations were readily analyzed by deposition directly on the platform, these reservoirs were necessary for less viscous, non-gel hand sanitizers, which tended to spread rapidly across the stainless-steel platform when deposited. Data acquisition was initiated immediately after sample deposition. Two consecutive repeat scans, without sample replacement, were collected for each analysis.

\section{Calibrants and Standards}

A series of calibration solutions for ethanol content were prepared based on the WHO/FDA recommendations that included only ethanol, glycerin, hydrogen peroxide, and water. The solution compositions were based on a volumetric target composition but prepared gravimetrically. Apart from water, the components were weighed into $50 \mathrm{~mL}$ volumetric flasks with target weights based on volume from established densities at $20^{\circ} \mathrm{C}$ [14]. No density value for $3 \%$ hydrogen peroxide could be found so the density of pure water was assumed. The glycerol and hydrogen peroxide targets were fixed at $1.45 \%$ (by volume) and $0.125 \%$ (by volume) for all calibrants while the ethanol content was varied from $50 \%$ to $90 \%$ (by volume) in $10 \%$ intervals with the balance made up with water for a total of five calibration solutions. After gravimetrically adding the different components to a volumetric flask, water (HPLC grade) was added to volume with the mass recorded. The solution temperature was slightly elevated at this point due to the enthalpy of mixing. The prepared solutions were subsequently allowed to return to ambient temperature before the final addition of water to achieve the calibrated volume. The lab temperature was monitored during preparation of calibrants and ranged from $19{ }^{\circ} \mathrm{C}$ to $20^{\circ} \mathrm{C}$. The mass of ethanol was converted to volume using the density of pure ethanol at $20^{\circ} \mathrm{C}$ and ratioed to the total solution volume to yield the ethanol content in volume $\%$ for each calibration solution.

\section{Quantitation}

ATR-FTIR spectra were acquired for the series of calibrant solutions to generate a calibration curve for ethanol content. The series of calibrants were run on several days when multiple hand sanitizer samples were being analyzed. The instrument configuration was not changed during this investigation, and the calibration was found to be very stable over time. When only a small number of samples were being analyzed, a single calibrant, $60 \%$ ethanol (by volume), was measured as a control to check for consistency with previous calibration data. To identify spectral bands for calibration purposes, pure component spectra were collected for the constituents in the calibrant formulations and for isopropanol, which could be present in significant quantities in some ethanol-based formulations as a denaturant. Figure 2 presents spectra of the five calibrants (left panel) as well as the pure component spectra of the constituents and isopropanol (right panel). For ethanol quantification purposes one of the C$\mathrm{O}$ stretching bands from $1020 \mathrm{~cm}^{-1}$ at $1065 \mathrm{~cm}^{-1}$ was selected. While this band is largely free from isopropanol interference, glycerin has significant overlapping absorbance in this region. Consequently, deviations in glycerin content from $1.45 \%$ volume, will have some impact on prediction accuracy. 
Band integrations were conducted without performing a baseline correction. A linear relationship was observed for ethanol content (volume \%), and a calibration was performed by linear regression of the peak area data (ethanol volume $\%=9.3863 \times$ area -16.8549 ; $\left.r^{2}=0.9998\right)$. Since no baseline was subtracted and other components exhibit broad absorbances underlying this band (particularly water), a zero-intercept was not expected.
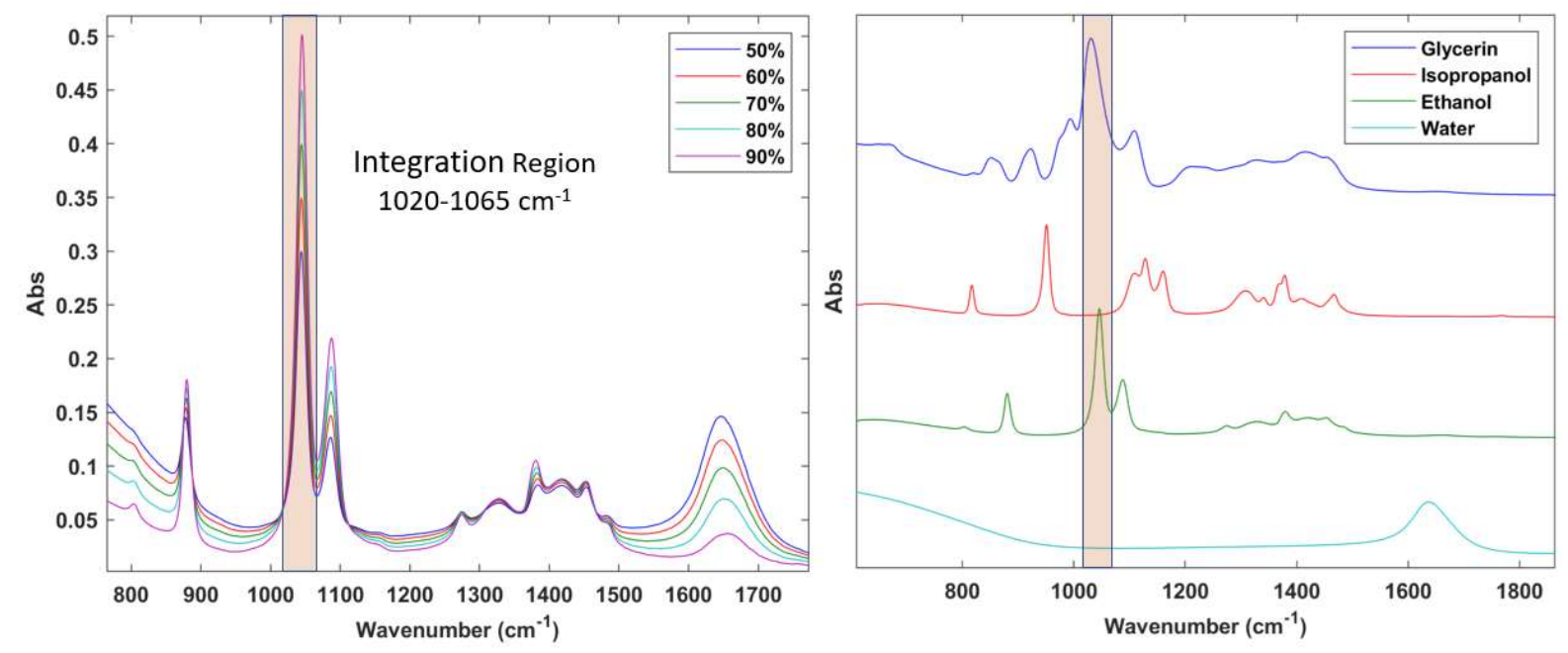

Figure 2. Spectra of calibrants used for the ATR-FTIR method.

\subsubsection{NMR Method}

NMR has a long history as a structure elucidation tool but is also a powerful quantitative analytical technique. Sample preparation is generally straightforward, and quantification of numerous components within a sample can be accomplished using a single reference compound of known purity, which can simplify method development. In this study, qNMR was used as a screening method to measure ethanol and other components using an internal standard approach. After the initial data collection for screening purposes, quantitative estimates of numerous impurities in many hand sanitizer samples were obtained using the intrinsic ethanol content in the hand sanitizers as a reference based on ATR-FTIR measurements. The estimates derived from this approach ultimately informed sample selection for further analysis by the chromatography-based techniques.

\section{Instrumental}

Hand sanitizer samples were analyzed using a Bruker Avance II $600 \mathrm{MHz}$ NMR spectrometer equipped with a 5-mm broadband inverse (BBI) probe and a Sample Xpress autosampler. Proton NMR was used with quantitation based on an internal standard $(1 \mathrm{H}-$ $\mathrm{qNMR}_{\mathrm{IS}}$ ). The pulse sequence consisted of a 90-degree excitation pulse width with inverse gated ${ }^{13} \mathrm{C}$ decoupling during acquisition (Bruker zgig pulse program). Typical instrumental acquisition parameters were the following: either 32 or 64 scans with 8 dummy scans, spectral sweep width of $20.0276 \mathrm{ppm}(12019.23 \mathrm{~Hz}), 131072$ complex data points, $5.45 \mathrm{~s}$ acquisition time, $45 \mathrm{~s}$ recycle delay, ${ }^{1} \mathrm{H}$ transmitter offset of $6.175 \mathrm{ppm}(3706 \mathrm{~Hz}),{ }^{13} \mathrm{C}$ transmitter offset of $35.00 \mathrm{ppm}(5281.60 \mathrm{~Hz})$. Decoupling was performed using the GARP4 
decoupling pulse sequence with the decoupling power level reduced (to minimize sample heating) and the ${ }^{13} \mathrm{C}$ transmitter offset set to effectively decouple the ${ }^{13} \mathrm{C}$ satellites on the ethanol ${ }^{1} \mathrm{H}$ peaks, which obscured minor components and impurities. All NMR experiments were performed at a sample temperature of approximately $25^{\circ} \mathrm{C}$.

\section{Calibrants and Samples}

Glassware used during sample preparation was rinsed with organic solvents and distilled water and placed in an oven at $110^{\circ} \mathrm{C}$ prior to use. All samples were prepared as dilutions in deuterium oxide $\left(\mathrm{D}_{2} \mathrm{O}\right.$; Cambridge Isotope Laboratory, DLM-4-25, D-99.9\%). For quantitative measurements based on addition of an internal standard, the calibrant used was either KHP or $\mathrm{DMSO}_{2}$. For KHP, internal standard stock solutions were prepared by weighing $0.5 \mathrm{~g}$ of KHP and $25 \mathrm{~g}$ of $\mathrm{D}_{2} \mathrm{O}$ gravimetrically into a $50 \mathrm{~mL}$ vial and thoroughly mixing. When $\mathrm{DMSO}_{2}$ was used, individual samples were prepared by weighing $10 \mathrm{mg}$ to 20 $\mathrm{mg}$ into sample vials followed by addition of $1 \mathrm{~mL}$ of $\mathrm{D}_{2} \mathrm{O}$. The use of $\mathrm{DMSO}_{2}$ was investigated after a precipitate was observed in a small number of samples in the presence of KHP. The sample constituents causing this effect were not investigated.

A total of 18 hand sanitizer samples were measured using an internal standard added for quantitative analysis. For most hand sanitizers, samples were analyzed without addition of an internal standard, and the content of minor components and impurities were estimated retrospectively by using the ${ }^{1} \mathrm{H}$ ethanol peak areas as the internal standard based on the intrinsic ethanol content of the hand sanitizer. This required an external estimate of ethanol content in the sample, which resulted from the ATR-FTIR analysis of the hand sanitizer.

Hand sanitizer samples (30 mg to $100 \mathrm{mg}$ ) were weighed directly into $1.85 \mathrm{~mL}$ screw top (PTFE lined) glass sample vials already containing $1 \mathrm{~mL}$ of $\mathrm{D}_{2} \mathrm{O}$ (with or without internal standard) and immediately sealed to minimize loss of any volatile components. After weighing, samples were vortex mixed and inspected to ensure complete mixing and dissolution. Depending on the viscosity of the hand sanitizer formulation, this process was repeated several times to ensure complete dissolution. In some gel formulations, the solution remained slightly turbid after mixing. Approximately $1 \mathrm{~mL}$ of sample was transferred into clean 600 MHz NMR tubes (Wilmad WG-1000-7-SJ, 5 mm diameter, 178 mm long).

For compound identification and peak assignment purposes, hand sanitizer spectra were compared to available spectral data of ingredients and potential impurities from various sources and then confirmed by comparisons to spectra of known standards. For these comparisons, samples of pure reference compounds of hand sanitizer ingredients and impurities (Table 2) were prepared by placing small quantities into $\mathrm{D}_{2} \mathrm{O}$ (Cambridge Isotope Laboratory, DLM-4-25, D-99.96\% with $0.01 \mathrm{mg} / \mathrm{mL} \mathrm{DSS}$ ). These were typically prepared by transferring a small drop by glass pipette into the solvent ampoule containing $0.7 \mathrm{~mL} \mathrm{D}_{2} \mathrm{O}$, mixing, and then transferring into an NMR tube for analysis.

\section{Quantitation}

For quantitative measurements, the mass \% of the primary chemical components (e.g., ethanol, isopropanol) or the levels of the impurities in the hand sanitizer samples, were calculated using the following equation: 


$$
\operatorname{mass} \%=\frac{N_{I}}{N_{C}} \times \frac{M_{C}}{M_{I}} \times \frac{A_{C}}{A_{I}} \times \frac{m_{I}}{m_{H S}} \times P_{I}
$$

where,

$$
\begin{aligned}
& c=\text { chemical component being determined } \\
& N_{\mathrm{C}}=\text { multiplicity (\# H atoms/peak) of the chemical component spectral peak } \\
& N_{\mathrm{I}}=\text { multiplicity (\# } \mathrm{H} \text { atoms/peak) of the internal standard peak } \\
& M_{\mathrm{C}}=\text { relative molar mass (molecular weight, } \mathrm{g} / \mathrm{mol} \text { ) of the chemical component } \\
& M_{I}=\text { relative molar mass (molecular weight, } \mathrm{g} / \mathrm{mol} \text { ) of the internal standard } \\
& A_{\mathrm{C}}=\text { integrated area of the chemical component peak } \\
& A_{I}=\text { integrated area of the internal standard peak } \\
& m_{\mathrm{HS}}=\text { mass }(\mathrm{g}) \text { of the hand sanitizer material } \\
& m_{I}=\text { mass }(\mathrm{g}) \text { of the internal standard } \\
& P_{I}=\text { purity (mass } \% \text { ) of the internal standard }
\end{aligned}
$$

The internal standard was KHP, $\mathrm{DMSO}_{2}$, or the intrinsic ethanol content in the hand sanitizer sample. For KHP, all aromatic protons were integrated together. For $\mathrm{DMSO}_{2}$ the single peak was used. For ethanol, the $-\mathrm{CH}_{3}$ and $-\mathrm{CH}_{2}$ - peaks were integrated separately. When glycerin was present in the sample, 2 proton signals are completely obscured beneath the ethanol $\mathrm{CH}_{2}$ - quartet. The ethanol integration was corrected for this interference by subtracting area counts based on the estimated glycerin content from one or both remaining peaks. Similarly, the isopropanol $-\mathrm{CH}_{3}$ peak is highly overlapped with the ethanol $-\mathrm{CH}_{3}$ peak, and a correction for this was made from an estimate of isopropanol content from the $-\mathrm{CH}$ - peak. After any required corrections for underlying peaks or other nearby impurity peaks, the areas of the two ethanol peaks were checked for consistency and used together to estimate the ethanol content or to serve as the internal standard peak area for estimating impurity content. For most impurities, mass \% results were converted to ppm $(\mu \mathrm{g} / \mathrm{g})$ to be consistent with FDA guidance.

\subsubsection{GC Methods}

Capillary GC has high selectivity and sensitivity for characterizing alcohols and impurity compounds $[8,10]$ and was used for the analysis of hand sanitizers. FID was selected as the predominant detection method for GC because it is simple to operate, robust, and provides excellent quantitative reproducibility. Additional qualitative measurements were conducted combining GC with mass spectrometric (MS) detection for identifying impurities in hand sanitizer samples.

\subsubsection{GC-FID}

\section{Instrumental}

GC-FID was used to analyze and quantitate alcohols and impurities in the hand sanitizer samples. Two separate GC-FID methods were developed to analyze ethanol or isopropanol, the active ingredients in the hand sanitizers that are present in the high percent range, and for impurities that may be present in the low $\mu \mathrm{g} / \mathrm{g}(\mathrm{ppm})$ range. The gas chromatograph used in 
both methods was an Agilent 7890A equipped with an autosampler and an FID detector. An RXI-1301 Sil MS column (Restek, Bellefonte, PA) with dimensions $60 \mathrm{~m}$ x $250 \mu \mathrm{m}$ x $1 \mu \mathrm{m}$ $\mathrm{d}_{\mathrm{f}}$ (film thickness) was used. Helium was used as the carrier gas at a flow rate of $1 \mathrm{~mL} / \mathrm{min}$. For both methods the FID temperature was set at $250{ }^{\circ} \mathrm{C}$. The hydrogen, air and makeup gas (nitrogen) for the detector were set to $34 \mathrm{~mL} / \mathrm{min}, 375 \mathrm{~mL} / \mathrm{min}$, and $29 \mathrm{~mL} / \mathrm{min}$, respectively.

For the determination of ethanol or isopropanol, the initial oven temperature was set to $40{ }^{\circ} \mathrm{C}$ for $1 \mathrm{~min}$ before ramping to $250^{\circ} \mathrm{C}$ at a rate of $10^{\circ} \mathrm{C} / \mathrm{min}$. The final oven temperature was held for $10 \mathrm{~min}$ for a total run time of $32 \mathrm{~min}$. The injection port temperature was $250{ }^{\circ} \mathrm{C}$ and a split liner (4 mm internal diameter) was used containing a silanized glass wool plug. A sample size of $0.4 \mu \mathrm{L}$ was injected using a $10 \mu \mathrm{L}$ syringe into a split/splitless injector with a split ratio of 100:1.

For the analysis of the impurities in the hand sanitizers, the initial oven temperature was set to $40{ }^{\circ} \mathrm{C}$ for $1 \mathrm{~min}$ before ramping to $250{ }^{\circ} \mathrm{C}$ at a rate of $5{ }^{\circ} \mathrm{C} / \mathrm{min}$. The final oven temperature was held for $5 \mathrm{~min}$ for a total run time of $48 \mathrm{~min}$. The injection port temperature was $275^{\circ} \mathrm{C}$ and a split liner ( $4 \mathrm{~mm}$ internal diameter) was used containing a silanized glass wool plug. A sample size of $0.4 \mu \mathrm{L}$ was injected using a $10 \mu \mathrm{L}$ syringe into a split/splitless injector with a split ratio of 50:1.

\section{Calibrants and Samples}

For determining ethanol content in non-gel hand sanitizers, samples were prepared gravimetrically by diluting $250 \mu \mathrm{L}$ of hand sanitizer and $250 \mu \mathrm{L}$ of an internal standard solution in $50 \mathrm{~mL}$ of water. The internal standard solution consisted of $60 \%-80 \%$ (by mass) $n$-propanol or acetonitrile in water. For gel hand sanitizers, samples were also prepared gravimetrically as described, but $5 \mu \mathrm{L}$ of concentrated $\mathrm{HCl}$ was added to the hand sanitizer sample and internal standard mixture to break the polymer crosslinking in the samples prior to dilution with water.

Calibration solutions for ethanol determinations were gravimetrically prepared in water from neat ethanol with known purity assessed at NIST for recent measurements using GC-FID and Karl Fischer analysis. For some analyses, the calibration solutions also included standards of neat methanol and isopropanol with purity estimated by GC-FID for this work (Table 2). For each measurement set, four to six independent calibrants were gravimetrically prepared that bracketed the anticipated alcohol content of the samples by combining $150-250 \mu \mathrm{L}$ of alcohol with $250 \mu \mathrm{L}$ of the same internal standard solution that was added to the hand sanitizer samples (60\% - $80 \%$ by mass n-propanol or acetonitrile) in $50 \mathrm{~mL}$ of water.

For determination of impurity content in non-gel hand sanitizers, samples were prepared gravimetrically by combining $100 \mu \mathrm{L}$ of an internal standard solution with $1 \mathrm{~mL}$ of a hand sanitizer sample. The internal standard solution was gravimetrically prepared by diluting $300 \mu \mathrm{L}$ of acetonitrile in $50 \mathrm{~mL}$ of dimethyl sulfoxide (DMSO) to yield a $\approx 4000 \mu \mathrm{g} / \mathrm{g}(\mathrm{ppm})$ solution. For gel hand sanitizers, $0.5 \mu \mathrm{L}$ of concentrated $\mathrm{HCl}$ was added to the hand sanitizer sample and internal standard to break the polymer crosslinking in the samples. After the addition of concentrated $\mathrm{HCl}$, the solution was vortex mixed. If the hand sanitizer solution did not liquify, then an additional $0.5 \mu \mathrm{L}$ of $\mathrm{HCl}$ was added. 
Impurities in the hand sanitizer samples were identified by comparing chromatographic retention times with standards prepared from neat chemicals, and the impurity content was estimated based on responses of the calibrants. For calibration of the chemical impurities in hand sanitizers, three independent mixed stock solutions were first prepared using neat chemicals with purity determined by GC-FID (Table 2) and DMSO as the solvent. Table 3 indicates the target volume of each impurity standard and DMSO that was added to prepare the stock solutions. Even though the solutions were prepared using volume targets, the masses were recorded to obtain the mass fraction of the impurities in each of the three stock solutions as listed in Table 3, which included correction for the purity of the neat chemicals. Nine additional intermediate stock solutions were gravimetrically prepared from the three independent stock solutions using appropriate dilutions with DMSO as the solvent to achieve a wide range in levels (also listed in Table 3). Finally, calibration solutions were prepared gravimetrically by adding $100 \mu \mathrm{L}$ of an internal standard solution (acetonitrile in DMSO described above) to $1 \mathrm{~mL}$ of an intermediate stock solution except for the highest-level intermediate stock solution, which was only used to prepare lower level intermediate stock solutions. Table 4 lists the final range of impurity levels $(\mu \mathrm{g} / \mathrm{g})$ in the calibration solutions used for quantitation.

Table 3. Preparation of stock and intermediate stock solutions for impurity compound analysis by GC-FID.

\begin{tabular}{|l|l|l|l|}
\hline $\begin{array}{l}\text { Impurities and } \\
\text { solvent }\end{array}$ & $\begin{array}{l}\text { Target volume } \\
\text { for preparation } \\
\text { of high-level } \\
\text { stock solutions } \\
(\boldsymbol{\mu L})\end{array}$ & $\begin{array}{l}\text { Nominal } \\
\text { mass fraction } \\
\text { (\%) of high- } \\
\text { level stock } \\
\text { solutions }\end{array}$ & $\begin{array}{l}\text { Nominal mass } \\
\text { fraction range of } \\
\text { intermediate-level } \\
\text { stock solutions } \\
\text { ( } \boldsymbol{\mu g} \text { /g) }\end{array}$ \\
\hline DMSO (solvent) & 3250 & NA & NA \\
Isobutanol & 3000 & 27 & 45 to 95000 \\
sec-Butanol & 900 & 7.8 & 13 to 28000 \\
Isoamyl & 600 & 5.2 & 9 to 19000 \\
alcohol & & 5.2 & 9 to 19000 \\
Amyl alcohol & 600 & 5.2 & 9 to 16000 \\
Acetone & 600 & 2.8 & 5 to 9800 \\
Ethyl acetate & 300 & 1.3 & 2 to 4500 \\
$n$-Butanol & 150 & 1.3 & 2 to 4500 \\
$n$-Propanol & 150 & 1.2 & 2 to 4400 \\
Methanol & 150 & 0.9 & 2 to 3300 \\
Benzene & 100 & 0.9 & 1 to 1200 \\
Acetal & 100 & 0.4 & \\
Acetaldehyde & 100 &
\end{tabular}




\section{Quantitation}

The mass \% of ethanol (also methanol and isopropanol in some samples) was determined using the average response factor (RF) of four to six calibrants. The RFs were determined using calibrants and were calculated using the following equation:

$$
R F=\frac{A_{S}}{A_{I S}} / \frac{C_{S}}{C_{I S}}
$$

where,

$$
\begin{aligned}
& A_{S}=\text { area of the alcohol } \\
& A_{I S}=\text { area of the internal standard } \\
& C_{S}=\text { mass } \% \text { of alcohol } \\
& C_{I S}=\text { mass } \% \text { of the internal standard }
\end{aligned}
$$

After the RF was determined from the calibrants, the same equation was used to determine the mass $\%\left(\mathrm{Cs}_{\mathrm{S}}\right)$ in the diluted hand sanitizer samples. Finally, the mass \% of the hand sanitizer was calculated by multiplying the mass $\%$ of the diluted hand sanitizer by the dilution factor.

For the analysis of hand sanitizer samples using the simple, non-gel WHO/FDA formulations, four independently prepared calibrants were prepared using $n$-propanol as the internal standard. The RFs that were generated from the four calibrants were comparable, and the percent relative standard deviation (\%RSD) of the average RF was low (0.32\% RSD). The average RF of the calibrants was used for quantifying the samples (RF of 1.063). Quantitation of alcohol in gel hand sanitizers were performed in the same manner as the WHO/FDA formulations. The average response factor ( 0.9748 with $0.39 \% \mathrm{RSD})$ was used to quantitate samples obtained from six independently prepared calibrants with acetonitrile as the internal standard.

The impurities were quantified using a calibration curve constructed from the ratio of the impurity to internal standard peak areas versus the ratio of the impurity to internal standard levels in $\mu \mathrm{g} / \mathrm{g}$ (ppm). Linear regression with a non-zero intercept was used. At least one calibrant prepared from each of the three independently prepared stock solutions were incorporated in the calibration curve. When possible, calibrant levels that bracketed the range of the FDA interim limits for the individual impurities were used. In some cases, the impurity content of a hand sanitizer sample was outside of the calibration range; in these cases, the value was marked indicating it was outside the calibration range. Table 4 lists the calibration curve information, including linear regression slope and intercept, and the calibration range for the impurities in the hand sanitizer samples. The limits of detections (LODs) are defined here as the lowest calibration point detected. For most impurities, this was a calibrant prepared about ten times lower that the lowest calibration point used for quantitation. 
Table 4. Calibration curve information for the impurities quantified by GC-FID.

\begin{tabular}{cccccc} 
Impurity & Calibration Range $(\mu \mathrm{g} / \mathrm{g})$ & $\mathrm{m}$ & $\mathrm{b}$ & $\mathrm{r}^{2}$ & $\begin{array}{c}\mathrm{LOD} \\
(\mu \mathrm{g} / \mathrm{g})\end{array}$ \\
\hline Acetaldehyde & $9-474$ & 0.59 & 0.0046 & 0.9992 & 8.1 \\
Methanol & $29-1154$ & 0.66 & 0.0063 & 1.0000 & 2.0 \\
Acetone & $121-4797$ & 0.98 & -0.0028 & 1.0000 & 9.2 \\
$n$-Propanol & $31-1277$ & 1.24 & -0.0056 & 0.9999 & 2.0 \\
Ethyl acetate & $67-2936$ & 0.85 & -0.0060 & 1.0000 & 4.7 \\
sec-Butanol & $185-7935$ & 1.27 & -0.0201 & 0.9999 & 13.4 \\
Isobutanol & $622-27124$ & 1.47 & -0.0570 & 0.9999 & 45.9 \\
Benzene & $22-886$ & 2.17 & -0.0069 & 0.9999 & 1.6 \\
$n$-Butanol & $31-1378$ & 1.36 & -0.0067 & 0.9999 & 2.2 \\
Acetal & $22-896$ & 1.03 & -0.0033 & 0.9999 & 1.5 \\
Isoamyl alcohol & $123-5299$ & 1.50 & -0.0170 & 0.9998 & 8.9 \\
Amyl alcohol & $125-5307$ & 1.48 & -0.0238 & 0.9998 & 9.0 \\
\hline
\end{tabular}

$\mathrm{m}=$ slope of linear regression; $\mathrm{b}=$ intercept of linear regression; $\mathrm{r}^{2}=$ coefficient of determination of linear regression; $\mathrm{LOD}=$ Limit of Detection

\subsubsection{GC-MS}

GC-MS was used for qualitative analysis of the hand sanitizer samples to identify unknown impurities, primarily through spectral matching to the NIST Library and by retention time matching of select calibrants that were prepared for GC-FID analysis.

\section{Instrumental}

GC-MS was used to confirm the identity of the impurities in select hand sanitizer samples. The instrument used was an Agilent 7890A gas chromatograph equipped with an autosampler coupled with an Agilent 5975c inert XL mass select detector. A J\&W Scientific DB-624 UI column with dimensions $60 \mathrm{~m} \times 250 \mu \mathrm{m} \times 1.4 \mu \mathrm{m} \mathrm{d}_{\mathrm{f}}$ (film thickness) was used. Helium was used as the carrier gas and was maintained at a constant flow rate of $1 \mathrm{~mL} / \mathrm{min}$. The MS source and quadrupole temperatures were set at $230^{\circ} \mathrm{C}$ and $150^{\circ} \mathrm{C}$, respectively. The MS was operated in scan mode between the mass to charge $(\mathrm{m} / \mathrm{z})$ range of $m / z 29$ to $\mathrm{m} / \mathrm{z} 200$. To protect the lifetime of the filament, the ion source and detector were turned off when ethanol (3.10-3.5 $\mathrm{min})$ and DMSO (23-28 $\mathrm{min})$ were eluting.

The initial oven temperature was set to $40^{\circ} \mathrm{C}$ for 5 min before ramping to $65^{\circ} \mathrm{C}$ at a rate of $2{ }^{\circ} \mathrm{C} / \mathrm{min}$. After reaching $65^{\circ} \mathrm{C}$, the oven was ramped to $240{ }^{\circ} \mathrm{C}$ at a rate of $10^{\circ} \mathrm{C} / \mathrm{min}$. The final oven temperature was held for $10 \mathrm{~min}$ for a total run time of $45 \mathrm{~min}$. The injection port temperature was $250^{\circ} \mathrm{C}$, and a split liner (4 mm internal diameter) was used containing a silanized glass wool plug. A volume of $0.4 \mu \mathrm{L}$ was injected using a $10 \mu \mathrm{L}$ syringe into a split/splitless injector with a split ratio of 50:1.

\section{Calibrants and Samples}

The calibration solutions and hand sanitizer samples for the qualitative GC-MS analyses were the same as prepared and described for the GC-FID measurements. 


\subsubsection{LC-UV Method}

Ethanol and isopropanol, as well as the harmful impurity methanol, have modest absorbance at $200 \mathrm{~nm}$. Thus, it was possible to develop a LC method with absorbance detection to identify and determine these alcohols in hand sanitizer products. However, LC-UV is not very sensitive and was only able to detect these alcohols at low percent levels. In addition, many of the other regulated impurities are also not strong UV absorbing compounds, so LC$\mathrm{UV}$ was not suitable for their detection in the $\mu \mathrm{g} / \mathrm{g}$ (ppm) range.

\section{Instrumental}

The LC system was comprised of a temperature-controlled autosampler and column compartment, binary pump, solvent degasser, and a fixed-wavelength UV detector. The method utilized a Phenomenex C18-2 with $5 \mu \mathrm{m}$ particles and $100 \AA$ pore size of dimensions $250 \mathrm{~mm}$ (length) $\times 4.6 \mathrm{~mm}$ (internal diameter). The column was maintained at a temperature of $15{ }^{\circ} \mathrm{C}$. Isocratic elution was used with a binary mobile phase comprised of $95 \%$ water, 5

$\%$ acetonitrile ( $\mathrm{vol} / \mathrm{vol})$ at a flow rate of $1.0 \mathrm{~mL} / \mathrm{min}$ to separate the three target alcohols. The total chromatographic analysis time was $10 \mathrm{~min}$. Detection was by UV absorbance at 200 $\mathrm{nm}$. Samples were placed in the temperature-controlled autosampler compartment maintained at $5{ }^{\circ} \mathrm{C}$. Injection volumes of $1 \mu \mathrm{L}$ were used to minimize peak tailing as well as fouling of the column by gel hand sanitizers. All chromatographic peak areas were determined by manual integration.

As most of the gel hand sanitizers have potential ingredients with greater retention than the alcohols, it was necessary to include a column flush cycle after the analysis of each gel sample. This was accomplished by injection of $100 \mu \mathrm{L}$ of methanol in conjunction with this isocratic separation. The flow rate was lowered to $0.2 \mathrm{~mL} / \mathrm{min}$ for $2 \mathrm{~min}$ and then the 1.0 $\mathrm{mL} / \mathrm{min}$ flow rate was resumed. A total chromatographic analysis time of $12 \mathrm{~min}$ was used for the flush cycle.

\section{Calibrants and Samples}

Calibrants were prepared by diluting neat standards of individual alcohols with water gravimetrically to mass percentages encompassing the levels expected in the hand sanitizers of $40 \%$ to $80 \%$ (by mass). In addition, SRM 2898 , nominally $25 \%$ (by mass) ethanol, was included in the calibration. Five ethanol calibrants were used for ethanol determination. Calibrants for methanol and isopropanol measurements were prepared at three levels covering the range of approximately $10 \%$ to $50 \%$ (by mass). Although prepared gravimetrically, the content of the ethanol and isopropanol calibration solutions were subsequently converted from mass $\%$ to volume $\%$ as will be discussed.

Hand sanitizer samples with non-gel consistency were analyzed directly after transfer to a 2-mL autosampler vial. Hand sanitizers with a gel consistency required additional sample preparation. A 2-mL autosampler vial with a cap was weighed and approximately $1 \mathrm{~mL}$ of hand sanitizer sample was transferred using a gas-tight syringe. The vial with cap and contents was weighed again. Next, $3 \mu \mathrm{L}$ of concentrated $\mathrm{HCl}$ was added to the sample to break the crosslinking of the gelling agent and produce a sample with much lower viscosity. The vial, cap, hand sanitizer sample, and $\mathrm{HCl}$ was weighed again. The vial was vortex mixed 
twice for $10 \mathrm{~s}$ to fully disperse the acid into the sample before sampling by LC-UV. The corrected mass of the sample, based on the additional mass of $\mathrm{HCl}$, was calculated and accounted for in the quantitative determinations of alcohol content.

\section{Quantitation}

For determinations by LC-UV, calibration required conversion of mass $\%$ to volume $\%$. This was required as the sample is injected by volume and the absorbance detector is linear with respect to volume $\%$. An internal standard was not used that could correct for volume effects. Mass \% was converted to volume $\%$ for ethanol and isopropanol water mixtures at $20{ }^{\circ} \mathrm{C}$ using a calculator obtained from https://handymath.com/calculators.html. A conversion table was not readily available for water-methanol mixtures. However, the limits for impurities are expressed in mass \% units (Table 1), and hence the methanol levels were calibrated in mass $\%$. Calibration plots of compound peak area versus alcohol \% were prepared from 3 injections of each calibration solution dispersed over the chromatographic determination sequence. Good linearity of response was obtained for the three alcohols (see Figure 3), and the equations of the curve-fit lines were determined.
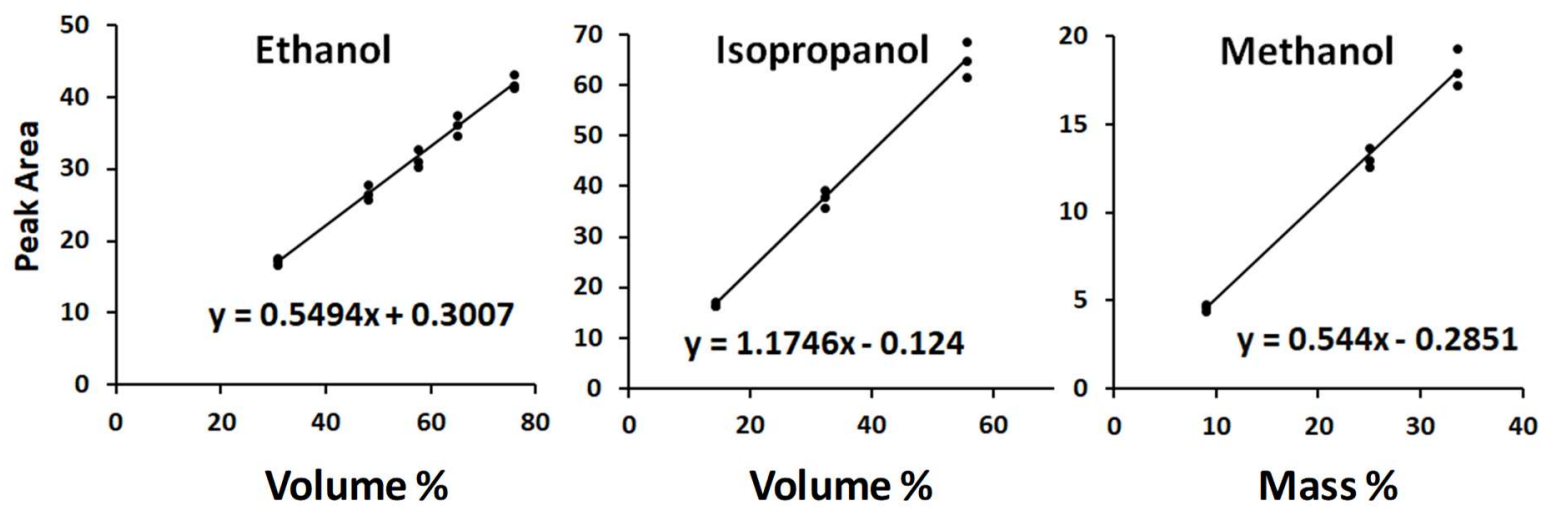

Figure 3. Calibration plots of LC chromatographic peak area versus alcohol \%.

Three injections were made for each hand sanitizer sample. The peak area (y-value) obtained for a sanitizer sample was then used to solve the equation for volume \% (ethanol and isopropanol) or mass \% (methanol) (x-value) from the corresponding curve-fit line shown above (Figure 3$)$. For example, the volume $\%$ ethanol $=($ ethanol peak area -0.3007$) / 0.5494$.

\subsubsection{Density}

The densities of the hand sanitizer samples were measured primarily to convert between mass $\%$ and volume \% for ethanol content. Expressing ethanol content in different units was necessary to enable comparison of the method results to each other and to the levels reported on the hand sanitizer product labels (see Appendix A). Density measurement was also explored as a potential approach for direct estimation of the ethanol content. This approach was based on a calibration curve generated from measuring the densities of the calibrants prepared according to the WHO/FDA formula that are detailed in the section describing the ATR-FTIR method. Determination of the density of gel hand sanitizers was often challenging because samples were viscous and often contained entrained air bubbles. To 
address these challenges, three methods for determining density were compared to assess applicability for these measurements.

\subsubsection{Lang-Levy Micropipette Method}

The density was measured in select hand sanitizers using a Lang-Levy micropipette method [15]. A nominal $200 \mu \mathrm{L}$ or $250 \mu \mathrm{L}$ pipette was calibrated by filling with pure water until the water reached the middle of the constriction of the Lang-Levy pipette using a precision suction device. The mass of water was determined, and the volume of the pipette was calculated based on the density of water at ambient laboratory conditions [13]. The calibrated volume of the pipette was determined as the average volume from triplicate determinations with water. In between replicates and samples, the Lang-Levy pipette was cleaned with water and ethanol and then dried using a vacuum line.

The density of select non-gel and gel hand sanitizers were determined using the Lang-Levy method. For each hand sanitizer, a sample was carefully drawn into the calibrated pipette to the middle of the constriction using a suction device, and the mass recorded. For gel samples, it was necessary to add $0.5 \mu \mathrm{L}$ of $\mathrm{HCl}$ to $1 \mathrm{~mL}$ of sample to render the samples less viscous to draw the sample into the pipette. Using the pipette volume and the sample mass, the density of the hand sanitizer was determined. The procedure was repeated with one or two more fresh samples, and the average of two or three determinations was calculated. The pipette was rinsed with water and ethanol between samples and dried using a vacuum line.

\subsubsection{Positive Displacement Pipette Method}

The density for all hand sanitizers was estimated using a positive displacement pipette. For this method, a $100 \mu \mathrm{L}$ positive displacement pipette was calibrated using three or more successive replicate water aliquots that were weighed into a pre-weighed receiving vial. The sample mass and the density of water at laboratory temperature [13] were used to determine the exact volume of the pipette.

The pipette at the same volume setting $(100 \mu \mathrm{L})$ was then used to aliquot successive replicates of a hand sanitizer sample that were weighed into a pre-weighed receiving vial. Care was taken to avoid sampling air bubbles that were present in some hand sanitizer samples. Prior to dispensing the sample into the pre-weighed receiving vial, excess sample was removed from the outside of the pipette tip. Based on the sample mass and the calibrated volume of the pipette, the density of the hand sanitizer sample was estimated.

\subsubsection{Hydrometer Method}

A hydrometer (ERTCO, USA), calibrated by the manufacturer for specific gravity at $15.55^{\circ} \mathrm{C}$ $\left(60^{\circ} \mathrm{F}\right)$, was evaluated for its suitability for use in hand sanitizer measurements. For the determination of specific gravity in non-gel hand sanitizer formulations, slightly more than $100 \mathrm{~mL}$ of the product was placed directly in a $100 \mathrm{~mL}$ graduated cylinder and capped with tightly fit aluminum foil. The cylinder was placed in a circulating water bath at $15.55^{\circ} \mathrm{C}$. A large paper wipe was placed over the top of the water bath to help achieve the desired temperature in the graduated cylinder. After approximately $30 \mathrm{~min}$ of thermal equilibration 
time, the hydrometer was carefully placed in the center of the cylinder. Some overflow of the product occurred, allowing the value of the hydrometer to be measured directly by floating above the top of the cylinder, rather than reading through the glass of the cylinder. A magnifier lens was used to estimate the value of the specific gravity. It was necessary for measurements of the gel sanitizers to render the samples less viscous by the addition of $200 \mu \mathrm{L}$ of concentrated $\mathrm{HCl}$. For these measurements, $50 \mathrm{~mL}$ of sanitizer was added to the cylinder, the $\mathrm{HCl}$ added, and then the final $50 \mathrm{~mL}$ was added. A long spatula was used to mix in the $\mathrm{HCl}$, breaking the gel properties. The effect of dilution of the sample by the $\mathrm{HCl}$ was neglected. The setup consisting of the temperature-controlled water bath, the graduated cylinder, and the hydrometer used for the determination of density is shown in Figure 4.

Specific gravity is defined here as the density of the hand sanitizer divided by the density of water at $15.55{ }^{\circ} \mathrm{C}$. Therefore, the hydrometer values were multiplied by the density of water at $15.55^{\circ} \mathrm{C}[16]$ to convert from specific gravity to density. The data were not corrected for the difference in specific gravity between room temperature (about $22^{\circ} \mathrm{C}$ ) versus $15.55^{\circ} \mathrm{C}$.

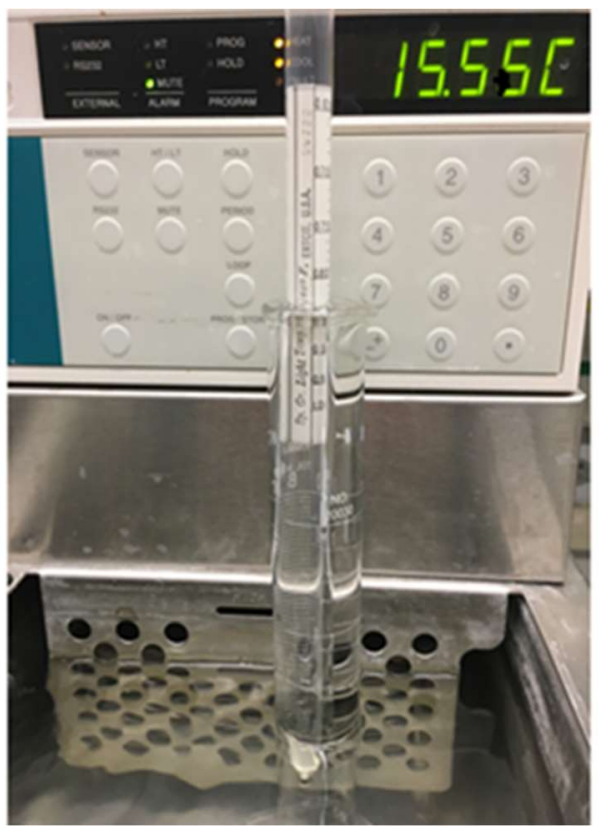

Figure 4. Determination of density of hand sanitizers with a hydrometer. 


\section{Results and Discussion}

Hand sanitizer samples were obtained from commercial sources (both online and physical retail stores). Exceptions included three materials that were provided to NIST by a local police department and two samples from dispenser stations. In total, 72 samples across 48 brands were obtained and analyzed as part of this work.

ATR-FTIR was used to screen and estimate the ethanol content in all hand sanitizers. In addition, NMR was used to screen most of the samples for ethanol and other ingredients and impurities. The results from these screening measurements, which are described in a subsequent section containing the individual method results (Section 3.3), partially impacted which hand sanitizers were analyzed by the additional methods GC-FID and LC-UV. The next two sections describe the results obtained for hand sanitizers containing percent levels of three alcohols ethanol, isopropanol, and methanol, which were identifiable by all methods evaluated for this work.

\subsection{Results for Ethanol by All Methods}

Ethanol was the primary alcohol disinfectant found in all but one of the hand sanitizers obtained for this study (see Appendix A). Four primary analytical methods qNMR, ATRFTIR, GC-FID, and LC-UV were used to determine the ethanol content in a subset of 18 hand sanitizers that were selected primarily because they were either: (1) from the local police department, (2) suspected to have ethanol content below $60 \%$ (by volume) based on ATR-FTIR results, (3) had notable amounts of impurities based on preliminary product evaluations using NMR, and/or (4) appeared to follow the temporary WHO/FDA manufacturing formula.

To facilitate a comparison of the measurement methods used in this study, the individual method results for ethanol reported as mass \% in selected hand sanitizers are presented in Table 5. Two of the methods, GC-FID and qNMR, were calibrated using solutions prepared in mass $\%$ and hence provided direct results for hand sanitizers in mass $\%$. The $\%$ RSD values for these two methods represent the measurement uncertainty for the hand sanitizer samples measured in replicate and was not determined for samples measured once. In contrast, the LC-UV and ATR-FTIR methods were calibrated using solutions converted to volume $\%$, and hence the mass \% results were derived from the volume \% results using the density of ethanol at $21{ }^{\circ} \mathrm{C}(0.7885 \mathrm{~g} / \mathrm{mL})$ and the hand sanitizer densities determined using the positive displacement pipette method (also provided in Table 5). The \%RSD for the derived results for LC-UV and ATR-FTIR include the relative uncertainties of the method and the hand sanitizer density measurements, as well as the estimated variability of the density of ethanol over the laboratory temperature range from $19{ }^{\circ} \mathrm{C}$ to $23{ }^{\circ} \mathrm{C}(\% \operatorname{RSD}=0.17 \%)$ [14].

The analogous results for ethanol in volume $\%$ are presented in Table 6 . For volume $\%$, the results for LC-UV and ATR-FTIR were directly calculated, whereas the results for GC-FID and qNMR were derived from the results in mass \% using the densities of ethanol and the hand sanitizers as described for the LC-UV and ATR-FTIR results in Table 5. Note that the ethanol results reported in volume \% (Table 6) are all greater than the results in mass \% (Table 5) for the same hand sanitizers, which is expected given the relationship between volume $\%$ and mass $\%$ for ethanol solutions (see Section 2.3 ). To facilitate a visual 
comparison of the individual method results, the values in Table 6 were plotted and are presented in Figure 5.

As can be observed in Tables 5 and 6 and Figure 5, good agreement was generally achieved across the methods for ethanol, except for the ATR-FTIR results for one hand sanitizer highlighted in red font (Sample 36-A) with a high percentage of methanol (methanol results are discussed in more detail in the subsequent section 3.2). For Sample 36-A, the ATR-FTIR was excluded from the calculations for the average ethanol content because the result was known to be biased. Even though sample viscosity impacted the sample preparation and handling for the chromatographic methods, it did not impact the comparability of the results across methods but did increase the variability of the results for the LC-UV method as observed by the high \%RSD (>5\%) for some gel samples. The individual method results for ethanol will be described in more detail in each of the follow subsections.

Table 6 also contains the average of the results from all methods, expressed as volume \% to facilitate a comparison to the CDC minimum and the label values. Given this was the only intention for the calculation of an average value, a rigorous uncertainty value for combining the different method results was not determined. The average results in Table 6 reveal that five of the 18 hand sanitizers contained less than the recommended $60 \%$ ethanol (by volume). A plot of the NIST average ethanol result (volume \%) versus the label claim for these hand sanitizers is provided in Figure 6. For visualization purposes, the NIST results are reported with error bars representing $\pm 2 \times \mathrm{SD}$, where the SD was calculated from the dispersion of individual method results in Table 6 for each hand sanitizer. For these 15 hand sanitizers, six were found to have less ethanol, five were found to have more, and four were consistent with the ethanol volume \% on the label. One hand sanitizer (Sample 6) was well below both the label claim and the $\mathrm{CDC}$ minimum of $60 \%$ by volume. 
Table 5. Results for ethanol (mass \%) in 18 hand sanitizers by four methods.

\begin{tabular}{|c|c|c|c|c|c|c|c|c|c|c|c|}
\hline & & & & & & $\mathrm{ETH} /$ & JOL RES & LTS: MA & $5 \%$ & & \\
\hline SA & MPLE & DEN & SITY & GC & & $\mathrm{LC}$ & & & & ATR- & TIR \\
\hline ID & Viscosity & $(\mathrm{g} / \mathrm{mL})$ & $\%$ RSD & $\begin{array}{l}\text { Direct } \\
\text { Result }\end{array}$ & $\% \mathrm{RSD}$ & $\begin{array}{c}\text { Derived } \\
\text { Result }\end{array}$ & $\%$ RSD & $\begin{array}{l}\text { Direct } \\
\text { Result }\end{array}$ & $\%$ RSD & $\begin{array}{c}\text { Derived } \\
\text { Result }\end{array}$ & $\%$ RSD \\
\hline 1 & non-gel & 0.848 & 0.11 & 76.6 & 0.3 & 77.8 & 1.5 & 76.3 & 0.1 & 76.1 & -- \\
\hline 2 & non-gel & 0.856 & 0.04 & 73.4 & 0.4 & 73.5 & 1.1 & 72.9 & 0.0 & 73.8 & -- \\
\hline 3 & non-gel & 0.852 & 0.12 & 71.3 & 0.3 & 71.1 & 1.6 & 70.9 & 0.6 & 71.6 & -- \\
\hline $4 \mathrm{~A}$ & gel & 0.834 & 0.09 & 54.4 & 0.4 & 60.3 & 9.2 & 54.0 & 0.1 & 56.7 & -- \\
\hline $4 B$ & gel & 0.845 & 0.09 & 54.5 & 0.3 & 60.0 & 5.8 & 54.2 & -- & 56.0 & -- \\
\hline 6 & gel & 0.957 & 0.18 & 27.2 & 0.3 & 28.5 & 22.3 & 27.0 & -- & 26.1 & 0.4 \\
\hline $7-C$ & gel & 0.869 & 0.07 & 69.0 & -- & 74.3 & 6.3 & 69.0 & -- & 69.0 & -- \\
\hline $8-A$ & gel & 0.925 & 0.09 & 43.7 & 0.8 & 45.3 & 11.1 & 43.5 & -- & 41.5 & 0.9 \\
\hline 10 & non-gel & 0.863 & 0.06 & 69.0 & 1.5 & 69.4 & 1.4 & 69.8 & -- & 70.6 & -- \\
\hline 12 & gel & 0.901 & 0.19 & 52.6 & 0.8 & 52.2 & 11.8 & 52.1 & 0.2 & 50.7 & 0.5 \\
\hline 13 & non-gel & 0.826 & 0.13 & 81.0 & 1.1 & 82.7 & 5.1 & 81.3 & -- & 80.9 & -- \\
\hline 17 & non-gel & 0.849 & 0.43 & 74.7 & 1.7 & 75.4 & 4.6 & 75.7 & -- & 76.3 & -- \\
\hline 20 & gel & 0.856 & 0.10 & 70.3 & -- & 67.4 & 9.1 & 70.7 & 0.1 & 68.5 & -- \\
\hline 30 & gel & 0.864 & 0.21 & 64.0 & -- & 65.5 & 8.3 & 64.2 & -- & 64.2 & -- \\
\hline $36-A$ & gel & 0.870 & 0.33 & 51.8 & 0.2 & 51.3 & 5.3 & 48.8 & -- & 63.9 & -- \\
\hline $36-B$ & gel & 0.874 & 0.06 & 65.7 & -- & 68.9 & 6.5 & 65.9 & 0.9 & 65.5 & -- \\
\hline 39-A-1 & gel & 0.881 & 0.16 & 61.8 & -- & 60.6 & 5.4 & 61.3 & 0.0 & 62.3 & -- \\
\hline 40 & non-gel & 0.845 & 0.43 & 75.2 & 1.8 & 76.3 & 1.0 & 75.3 & 0.1 & 75.5 & -- \\
\hline
\end{tabular}

--, \%RSD not determined for single measurements; Red font indicates result biased by a known interference

Table 6. Results for ethanol (volume \%) in 18 hand sanitizers by four methods along with the average method result for comparison to the CDC minimum value and the product label.

\begin{tabular}{|c|c|c|c|c|c|c|c|c|c|c|c|c|c|}
\hline & & & & & & & THANOL & RESULTS: & /OLUM & & & & \multirow{2}{*}{ LABEL } \\
\hline \multicolumn{2}{|c|}{ SAMPLE } & \multicolumn{2}{|c|}{ DENSITY } & \multicolumn{2}{|c|}{ GC-FID } & \multicolumn{2}{|c|}{ LC-UV } & \multicolumn{2}{|c|}{ qNMR } & \multicolumn{2}{|c|}{ ATR-FTIR } & AVERAGE & \\
\hline ID & Viscosity & $(\mathrm{g} / \mathrm{mL})$ & \%RSD & $\begin{array}{l}\text { Derived } \\
\text { Result }\end{array}$ & $\%$ RSD & $\begin{array}{l}\text { Direct } \\
\text { Result }\end{array}$ & $\%$ RSD & $\begin{array}{l}\text { Derived } \\
\text { Result }\end{array}$ & \%RSD & $\begin{array}{l}\text { Direct } \\
\text { Result }\end{array}$ & $\%$ RSD & $\begin{array}{c}\text { All } \\
\text { Methods }\end{array}$ & Vol. \% \\
\hline 1 & non-gel & 0.848 & 0.11 & 82.3 & 0.5 & 83.6 & 1.3 & 82.0 & 0.4 & 81.8 & -- & 82.4 & $\mathrm{~N} / \mathrm{A}$ \\
\hline 2 & non-gel & 0.856 & 0.04 & 79.7 & 0.6 & 79.8 & 0.9 & 79.1 & 0.2 & 80.1 & -- & 79.7 & N/A \\
\hline 3 & non-gel & 0.852 & 0.12 & 77.0 & 0.6 & 76.8 & 1.3 & 76.6 & 0.9 & 77.4 & -- & 76.9 & N/A \\
\hline $4 \mathrm{~A}$ & gel & 0.834 & 0.09 & 57.5 & 0.7 & 63.8 & 9.0 & 57.1 & 0.3 & 60.0 & -- & 59.6 & 70 \\
\hline $4 B$ & gel & 0.845 & 0.09 & 58.4 & 0.6 & 64.2 & 5.5 & 58.1 & -- & 60.0 & -- & 60.2 & 70 \\
\hline 6 & gel & 0.957 & 0.18 & 33.0 & 0.6 & 34.6 & 21.9 & 32.8 & -- & 31.7 & 0.1 & 33.0 & 70 \\
\hline $7-C$ & gel & 0.869 & 0.07 & 76.0 & -- & 81.8 & 6.1 & 76.0 & -- & 76.0 & -- & 77.4 & 70 \\
\hline $8-A$ & gel & 0.925 & 0.09 & 51.3 & 1.0 & 53.1 & 10.8 & 51.0 & -- & 48.7 & 0.6 & 51.0 & 65 \\
\hline 10 & non-gel & 0.863 & 0.06 & 75.5 & 1.8 & 75.9 & 1.1 & 76.3 & -- & 77.2 & -- & 76.2 & 80 \\
\hline 12 & gel & 0.901 & 0.19 & 60.1 & 1.1 & 59.6 & 11.5 & 59.5 & 0.5 & 58.0 & 0.1 & 59.3 & 75 \\
\hline 13 & non-gel & 0.826 & 0.13 & 84.8 & 1.4 & 86.6 & 4.8 & 85.2 & -- & 84.7 & -- & 85.3 & 80 \\
\hline 17 & non-gel & 0.849 & 0.43 & 80.4 & 2.3 & 81.2 & 4.0 & 81.5 & -- & 82.2 & -- & 81.3 & 80 \\
\hline 20 & gel & 0.856 & 0.10 & 76.3 & -- & 73.2 & 8.8 & 76.7 & 0.3 & 74.3 & -- & 75.1 & 70 \\
\hline 30 & gel & 0.864 & 0.21 & 70.1 & -- & 71.7 & 7.9 & 70.4 & -- & 70.4 & -- & 70.7 & 62 \\
\hline $36-A$ & gel & 0.870 & 0.33 & 57.1 & 0.7 & 56.6 & 4.8 & 53.8 & -- & 70.5 & -- & $55.9 *$ & 70 \\
\hline $36-B$ & gel & 0.874 & 0.06 & 72.8 & -- & 76.4 & 6.2 & 73.0 & 1.1 & 72.6 & -- & 73.7 & 70 \\
\hline 39-A-1 & gel & 0.881 & 0.16 & 69.0 & -- & 67.7 & 5.0 & 68.5 & 0.4 & 69.7 & -- & 68.7 & 70 \\
\hline 40 & non-gel & 0.845 & 0.43 & 80.6 & 2.4 & 81.8 & 0.4 & 80.7 & 0.7 & 80.9 & -- & 81.0 & 80 \\
\hline
\end{tabular}

--, \%RSD not determined for single measurements; Red font indicates result biased by a known interference; ${ }^{*}$, average excludes ATR-FTIR result. 

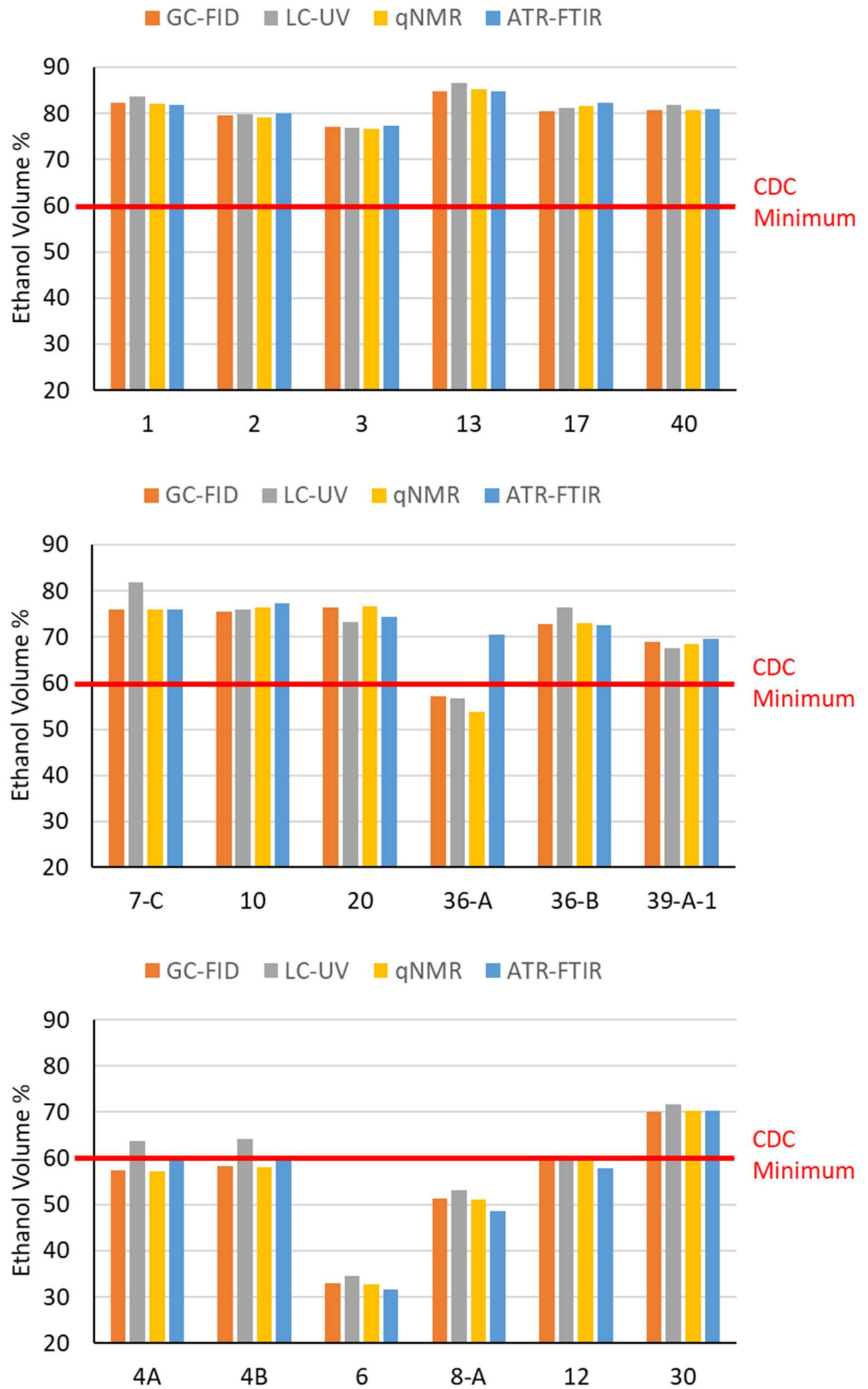

Figure 5. Plots of NIST method results for ethanol (volume \%) in select hand sanitizers. 


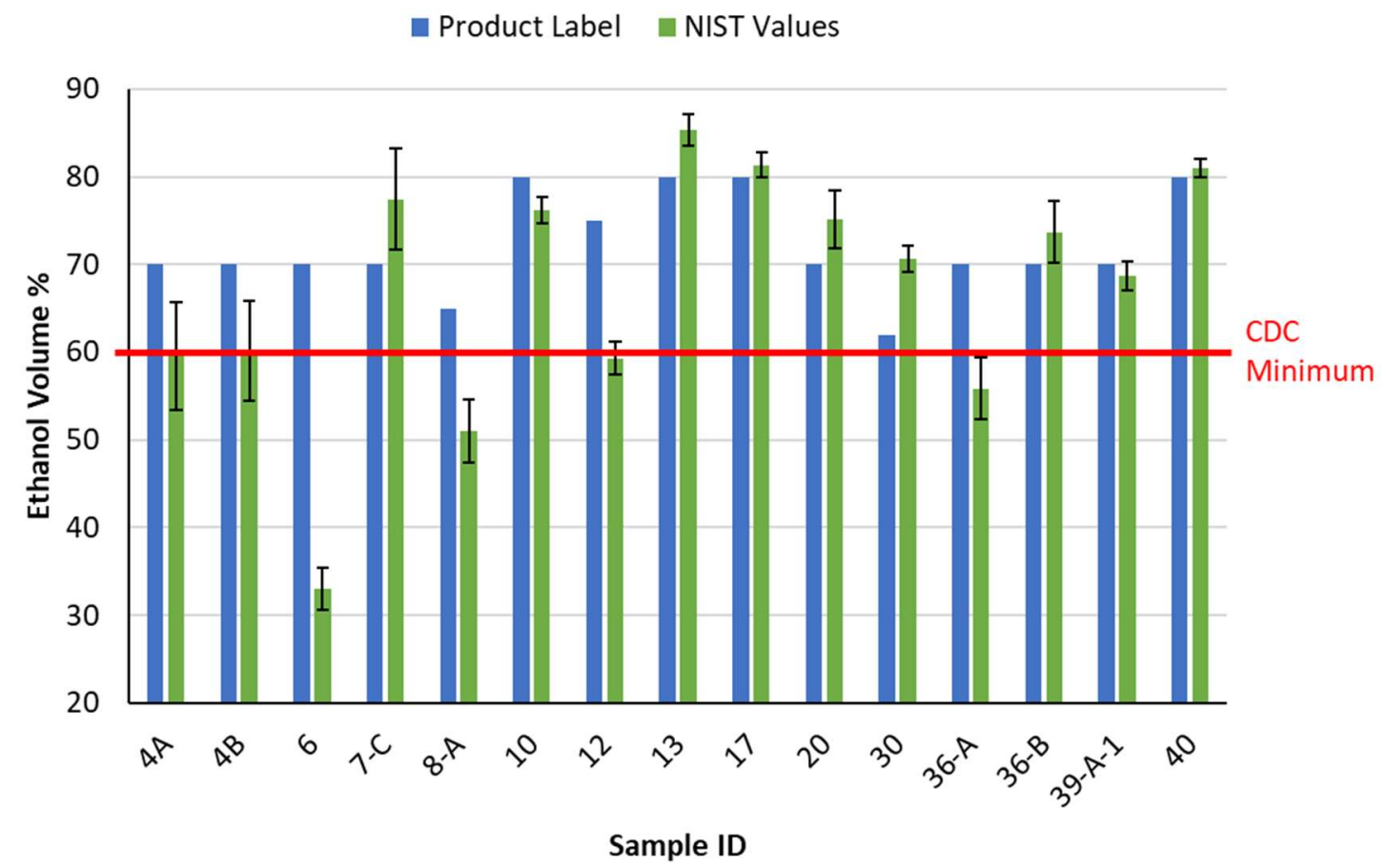

Figure 6. Comparison of the NIST average result to the product label claim for ethanol volume $\%$.

\subsection{Results for Methanol and Isopropanol by Multiple Methods}

Given the prevalence of hand sanitizers recalled by the FDA due to contamination with methanol, we focused special attention on the identification and quantitation of this impurity in hand sanitizers. Methanol is currently regulated to a limit of $630 \mathrm{ppm}(0.063$ mass \%) under the interim FDA guidance (see Table 1).

Hand sanitizer 36-A was qualitatively identified as containing a high level of methanol by all four methods investigated for this work. The mass \% for methanol in Sample 36-A was quantitated using GC-FID, LC-UV, and qNMR, with results presented in Table 7; the ATRFTIR method was not developed to quantitate methanol and hence no value was determined. The three quantitative methods were calibrated using solutions prepared in mass $\%$ and hence provided direct results for methanol in hand sanitizers in mass \%. The GC-FID and qNMR results for this sample agree, but the LC-UV results are somewhat low and have a large variability (11\%RSD). For the LC-UV method, Sample 36-A was cloudy and required filtering after adding $\mathrm{HCl}$, which could contribute to the higher variability and poorer agreement with the other results.

In addition to Sample 36-A, screening results from NMR identified four additional hand sanitizers (Sample 13, Sample 17, Sample 31, and Sample 36-B) that appeared to contain methanol above the FDA limit of $0.063 \%$ (by mass). When these samples were measured using a more rigorous approach by GC-FID, three of the four were found to exceed the limit 
(Sample 13, Sample 31, and Sample 36-B). Table 7 summarizes the results for these four additional hand sanitizer samples. The GC-FID and qNMR results agree except for Sample 17; the source of this discrepancy was not further explored. The four samples with lower levels of methanol were well below the detection limit for the LC-UV method $(\approx 1 \%$ by mass) and were not determined.

The results for methanol in Sample 36-A and Sample 36-B should also be compared to each other. Both samples were from the same brand (brand code 36) but represented different bottles and lot numbers (A and B). While both samples were found to exceed the FDA limit for methanol, Sample 36-A exceeds the FDA limit by a factor of over 200, whereas Sample 36 -B is very close to the limit at $\approx 0.07 \%$ (by mass).

Table 7. Summary of results for methanol (mass \%) in 5 hand sanitizers by three methods.

\begin{tabular}{|cc|cc|cc|cc|}
\cline { 3 - 8 } \multicolumn{1}{c|}{} & \multicolumn{5}{c|}{ METHANOL RESULTS: MASS \% } \\
\hline \multicolumn{2}{|c|}{ SAMPLE } & \multicolumn{2}{|c|}{ GC-FID } & \multicolumn{2}{c|}{ LC-UV } & \multicolumn{2}{c|}{ qNMR } \\
\hline \multirow{2}{*}{ ID } & \multirow{2}{*}{ Viscosity } & Direct & \%RSD & Direct & \%RSD & Direct & \multirow{2}{*}{ \%RSD } \\
& Result & & Result* & & Result & \\
\hline 13 & non-gel & 0.79 & -- & nd & -- & 0.76 & -- \\
17 & non-gel & 0.021 & -- & nd & -- & 0.065 & -- \\
31 & gel & 0.81 & -- & nd & -- & 0.73 & -- \\
$36-A$ & gel & 13.8 & 1.1 & 10.1 & 11 & 13.0 & -- \\
$36-B$ & gel & 0.072 & -- & nd & -- & 0.065 & -- \\
\hline
\end{tabular}

nd, not determined; --, \%RSD not determined for single measurements;

*LC-UV results calibrated by mass $\%$ for this impurity

Only one of the hand sanitizers evaluated in this study, Sample 44, was found to contain isopropanol as the primary disinfectant alcohol. In addition, isopropanol is an approved denaturant and was identified in seven additional products at much lower levels using qNMR (results to be discussed in Section 3.3.2). Results for isopropanol in volume \% in Sample 44 and five other hand sanitizers that met one of our four criteria for additional analyses by multiple methods are presented in Table 8. For isopropanol, the GC-FID and qNMR results were derived from results in mass \% whereas LC-UV yielded direct results for volume $\%$ (see descriptions of direct and derived results in Section 3.1). The results agree across the different methods and demonstrate the applicability of these measurement approaches for determining isopropanol in hand sanitizers. Since the gel hand sanitizers pose an additional measurement challenge for the LC-UV method, results were not determined for three of the hand sanitizers in Table 8 . 
Table 8. Summary of results for isopropanol (volume \%) in 6 hand sanitizers by three methods.

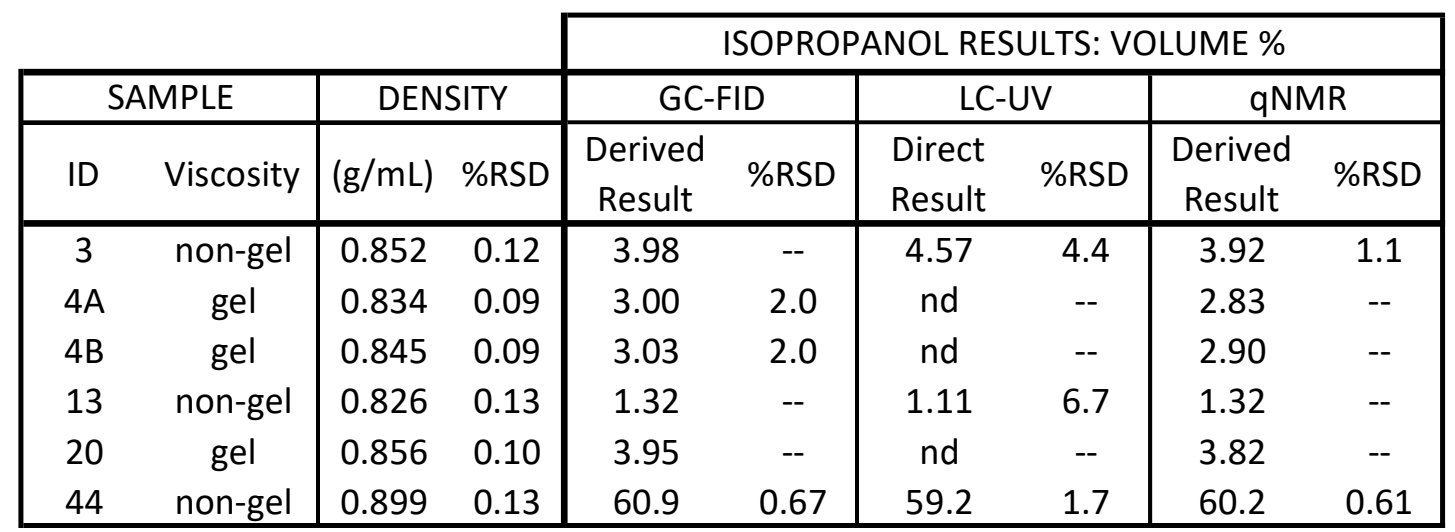

nd, not determined; --, \%RSD not determined for single measurements

\subsection{Individual Methods Results and Discussion}

As was just discussed, the GC-FID, qNMR, ATR-FTIR, and LC-UV methods used at NIST were tailored for the measurement of ethanol, the principle active ingredient in hand sanitizers. In addition, the three methods GC-FID, qNMR, and LC-UV were used to measure isopropanol and methanol in select samples. Isopropanol is of interest as an alternate disinfectant or a denaturant in hand sanitizers, and methanol is an impurity of concern.

The applicability of each method for determining additional chemical impurities (besides methanol) relative to the interim limits reported in the FDA guidance [4] and Table 1 of this report was investigated. Of the NIST methods, GC-FID had the selectivity and sensitivity to measure all potential impurities at the required levels, while qNMR was able to determine most of the compounds. The LC-UV method did not have the selectivity or sensitivity to measure the impurities except for methanol in one hand sanitizer (Sample 36-A) that contained a very high level of methanol. The use of ATR-FTIR for measuring any compounds besides ethanol was not explored for this work.

A comparison of the collective results for ethanol, methanol, and isopropanol were discussed in Sections 3.2 and 3.3; however, the details for each of the methods including any specific method challenges or strengths were not discussed. This Section (3.3) describes the individual method results in more detail. Additionally, the qNMR and GC-FID results for impurities are also presented and discussed.

\subsubsection{ATR-FTIR Results}

ATR-FTIR provided the simplest and fastest method for screening the ethanol content in hand sanitizers of the methods evaluated. ATR-FTIR was not used to determine impurities, many of which had regulated limits that were anticipated to be below reliable quantification or detection limits for the method utilized here. 
The method was used to estimate the ethanol content in each of the hand sanitizer materials listed in Appendix A. Spectra of Sample 39-A-1 and Sample 36-A are provided in Figure 7 along with spectra for an ethanol calibrant, pure methanol, and pure ethanol. Sample 39-A-1 was selected because it represents a 'typical' hand sanitizer containing primarily ethanol as the disinfectant with no other major alcohols present. Conversely, Sample 36-A was found to contain $\approx 13 \%$ (by mass) methanol in addition to ethanol as the primary disinfectant, as indicated by the shoulder on the primary ethanol peak used for integration and quantitation. For hand sanitizers such as Sample 36-A that contain a significant proportion of methanol, the spectral overlap of the methanol peak with the integration region used for ethanol (as illustrated for pure ethanol and methanol in Figure 7) contributes to a positive bias for the ethanol content estimated using the method described in this report. For Sample 36-A, methanol is readily observable as a shoulder on the ethanol peak. Other bands associated with methanol are also evident in this sample in regions of the spectrum not shown in Figure 7.

The estimated ethanol volume $\%$ for each of the hand sanitizers using single ATR-FTIR measurements is provided in Figure 8. The results in Figure 8 indicate that most materials screened in this study have ethanol content above the CDC recommended minimum of $60 \%$ (by volume), except for the five hand sanitizers labeled and highlighted in orange. Sample 36-A, which has $\approx 13 \%$ methanol (by mass), has an ethanol content that is overpredicted using the ATR-FTIR method (highlighted in yellow) due to the spectral overlap previously described.

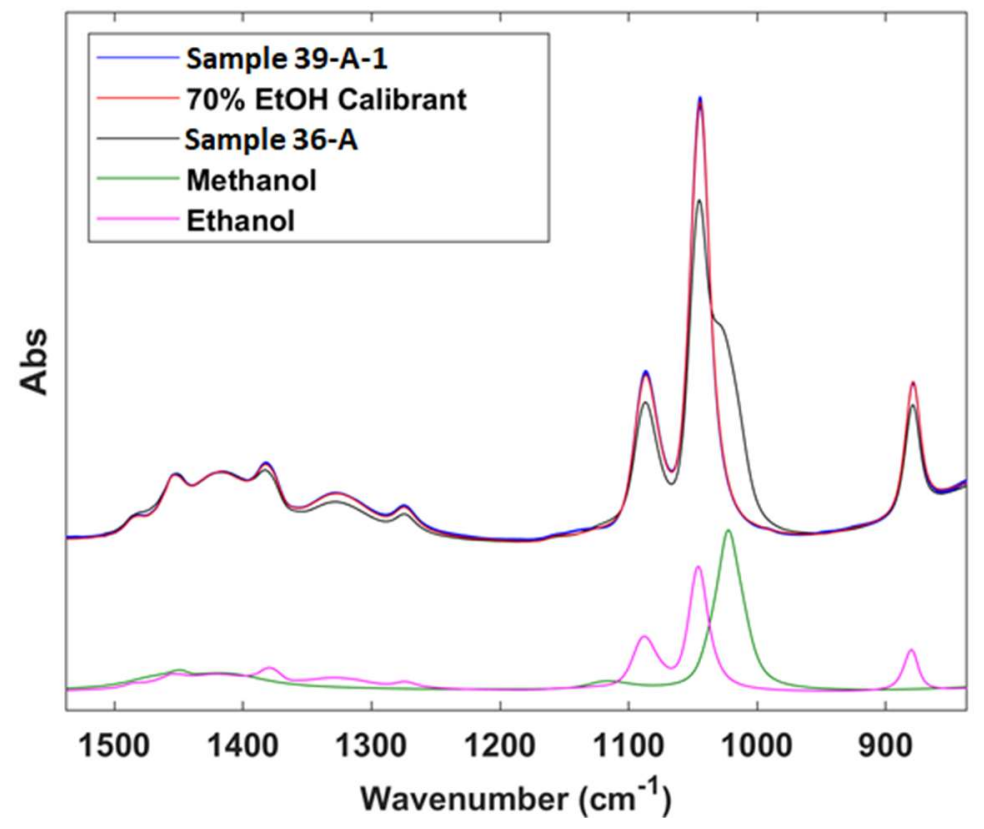

Figure 7. ATR-FTIR spectra of hand sanitizers compared to ethanol and methanol. 


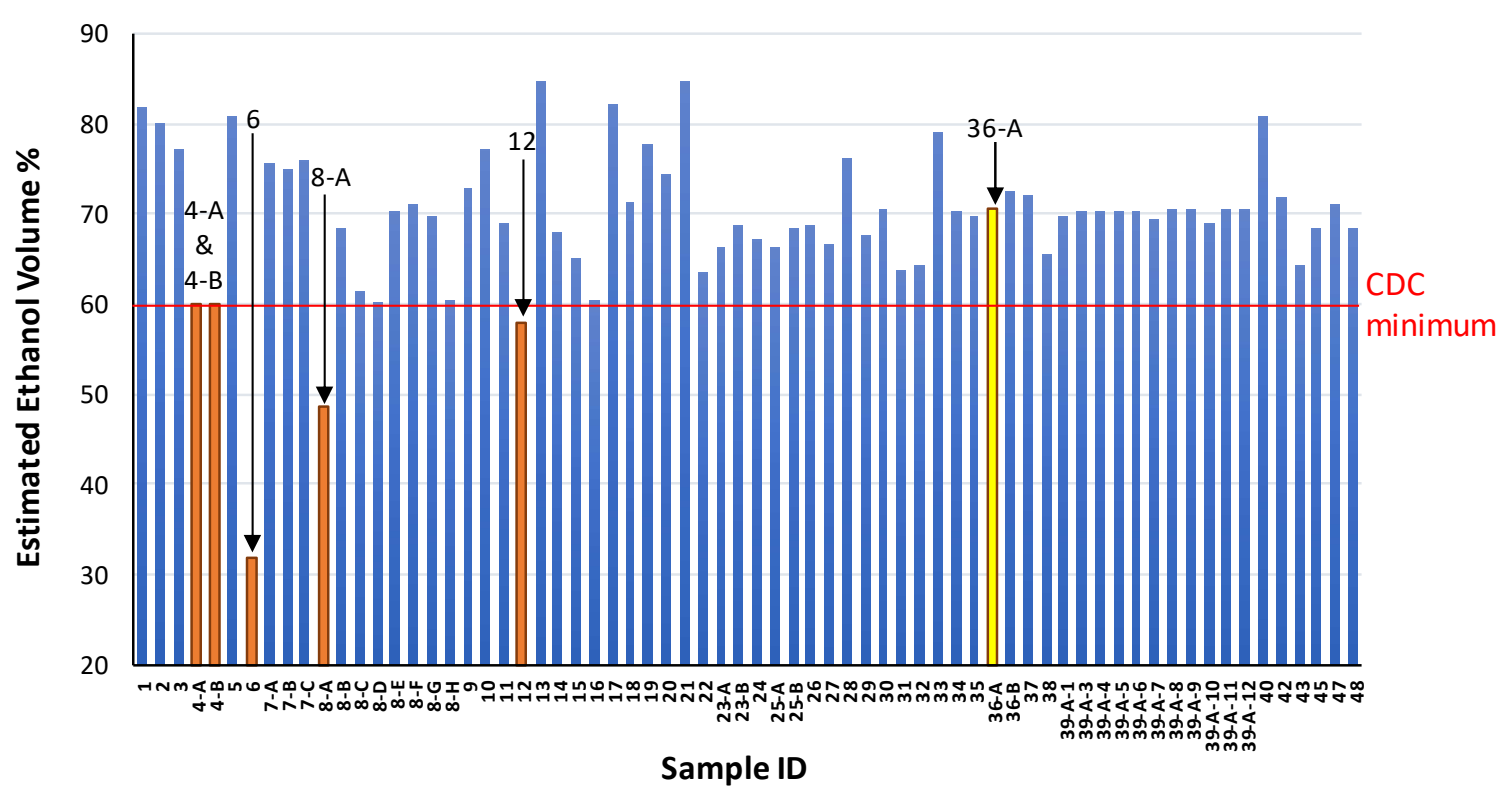

Figure 8. Estimated ethanol volume \% in hand sanitizers by ATR-FTIR.

The ATR-FTIR method is rapid, relatively easy, and is generally applicable to most hand sanitizers regardless of sample complexity. A notable exception where FTIR results did not agree well with the other method results is for a hand sanitizer sample that contained a significant amount of methanol. However, the presence of methanol in this sample was readily observed. Further method development to quantify methanol and establish detection limits is feasible but was not investigated here.

The applicability for the analysis of impurities is another potential limitation of the method. While the regulated levels of some impurities are likely to be in a detectable range, establishing reliable limits of detection considering all potential interferences (including both ingredients and other impurities) would require a thorough investigation. Finally, only a single hand sanitizer sample was obtained with isopropanol as the primary disinfectant and, consequently, the development of a quantitative method was not pursued. Based on the spectral features, a calibration model to quantify isopropanol should be feasible, either as the primary component or as a denaturant in ethanol-based formulations.

\subsubsection{NMR Results}

NMR was used both as an analytical method for the quantitative determination of ethanol and as a screening method for estimating the presence of denaturants and impurities in hand sanitizers. NMR can be used to screen hand sanitizers because it is straightforward and involved the simple dilution of hand sanitizer samples in $\mathrm{D}_{2} \mathrm{O}$. An example spectrum obtained for a hand sanitizer sample is shown in Figure 9. The spectrum exhibits moderate complexity but most components of interest, both ingredients and impurities, still exhibit one or more proton resonances with suitably resolved chemical shifts, $\delta$ (in ppm units), to enable identification and quantification. As indicated by the labels in Figure 9, ingredients such as 
isopropanol (denaturant) and glycerin (wetting agent) are readily discernible by NMR as are various impurities. Impurities that are spectrally resolved and that can potentially be quantified using NMR include methanol, acetaldehyde, acetal, ethyl acetate, benzene, acetone, isobutanol, isoamyl alcohol, and $n$-propanol. Additional impurity signals not shown in this figure included the aldehyde proton from acetaldehyde, observed as a quartet of resonances at a chemical shift of $9.66 \mathrm{ppm}$, and the - $\mathrm{CH}$ - proton from acetal, observed as a quartet at a chemical shift of $5.23 \mathrm{ppm}$. Acetal has 3 additional proton signals; however, none were detected, likely due to overlap with ethanol or other nearby signals.

Acetal, which is in equilibrium with acetaldehyde, was detected in most samples in which acetaldehyde was found but was not quantified due to poor signal-to-noise ratios and some spectral overlap with interfering peaks. Visual examination of the signals showed that the acetal content was directly correlated to acetaldehyde content in the sample. Peak fitting techniques may be necessary to provide suitable quantitative estimates in the case of acetal. These techniques may also offer improved quantitative accuracy and correction for interfering peaks in some other components as well, but this approach was not investigated.

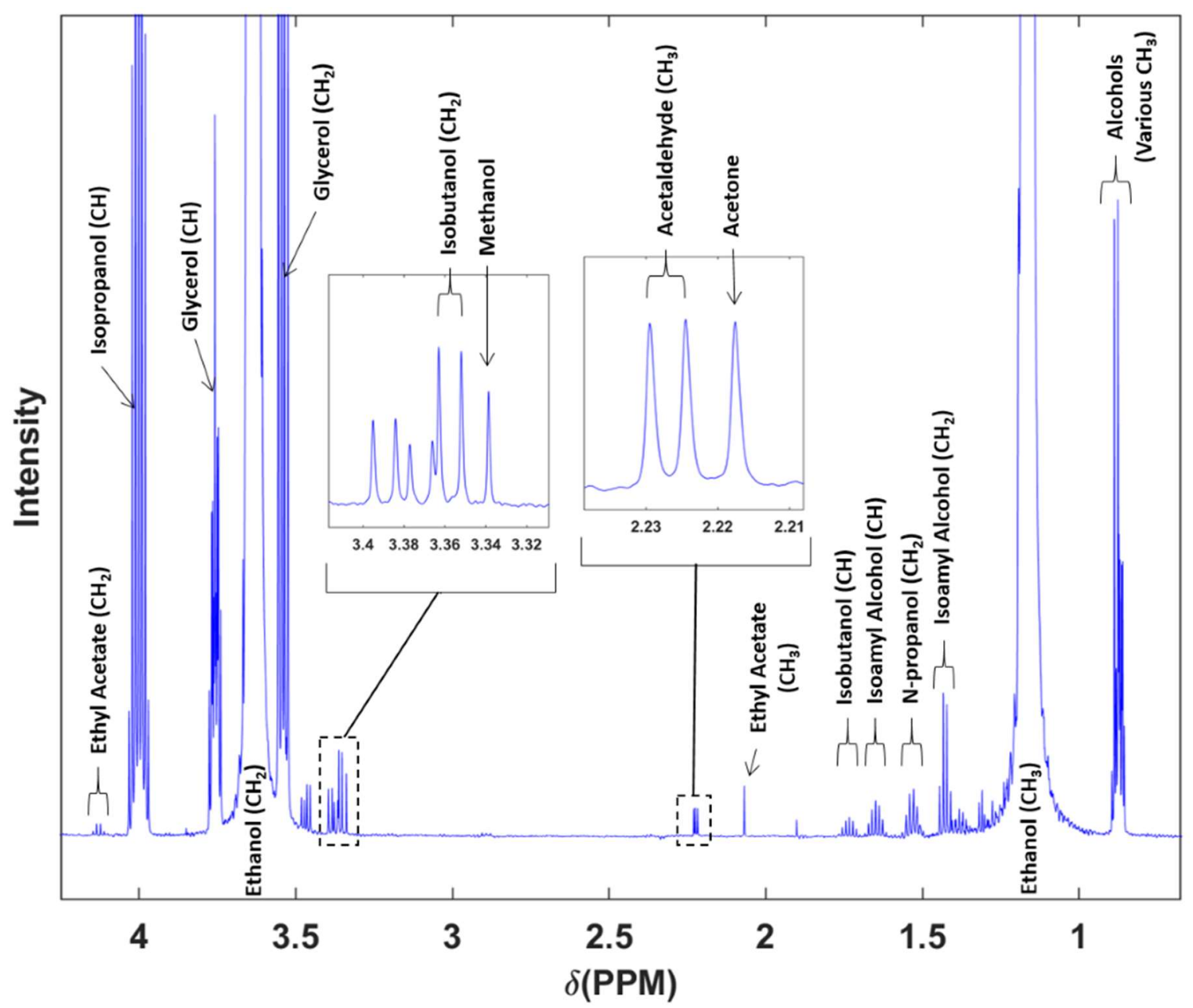

Figure 9. Example NMR spectrum of a hand sanitizer (Sample 2) showing ethanol, other ingredients, and impurities. 
The FDA limit for benzene of $2 \mathrm{ppm}(\mu \mathrm{g} / \mathrm{g})$ was below the detection limit for the NMR method used here. Benzene was detected with reasonable confidence in several hand sanitizers with a single sample (Sample 31 ) having a notably higher amount than any other, estimated at approximately $60 \mu \mathrm{g} / \mathrm{g}$ (ppm) by GC-FID (results to be discussed). Identification in the NMR spectrum was only tentative in several other samples that were found to have benzene levels below $10 \mu \mathrm{g} / \mathrm{g}$ (ppm) by GC-FID.

Quantitative NMR was used to determine the ethanol content in hand sanitizers using either potassium hydrogen phthalate or dimethyl sulfone as internal standards. Evaluated samples included three from the local police department (Sample 1, Sample 2, and Sample 3) and several commercial samples, including any that appeared to have less than the recommended $60 \%$ ethanol (by volume) (based on the ATR-FTIR screening results) or that appeared to have significant amounts of the regulated impurities. A summary of the qNMR results for ethanol in selected hand sanitizers was provided in Tables 5 and 6. qNMR is a sensitive, selective technique for determining ethanol and provided results with low variability that agreed well with the other method results.

Most of the hand sanitizers obtained for this study were analyzed for ingredients and impurities using the NMR screening approach. This data was subsequently used to estimate the impurity content in the hand sanitizers using the intrinsic ethanol content estimated in the sample as a quantitative internal reference. The estimated mass \% of ethanol in the sample was from either an existing qNMR result when available, or from ATR-FTIR. Two ingredients, isopropanol and tert-butanol, which are added to hand sanitizers as denaturants and are present at percent levels, were also estimated in 53 of the hand sanitizers using NMR (Figure 10). However, in many hand sanitizers, the compounds were either below the detection or quantitation limit; these results were estimated as $\approx 0 \%$ (by mass) for visualization purposes. Quantitative NMR results for isopropanol in select hand sanitizers were also presented in Table 8 and discussed in Section 3.3.

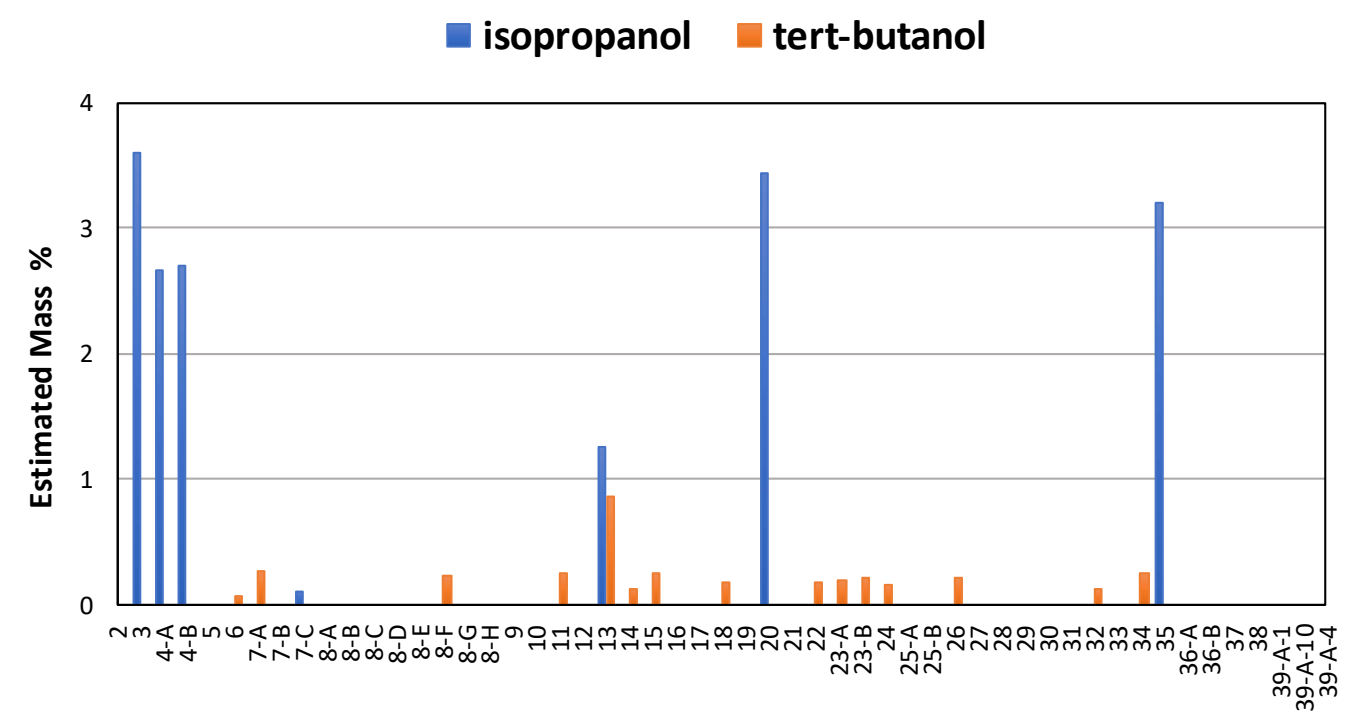

Figure 10. NMR estimates of denaturants in hand sanitizers. 
NMR was also used to estimate levels of impurities present in the hand sanitizer suite and to compare these results to the interim limits set by the FDA. Methanol, acetaldehyde, and $n$ propanol were the impurity compounds estimated to be above the FDA limit in some hand sanitizers using NMR (Figure 11). Isobutanol, isoamyl alcohol, acetone, and ethyl acetate were detected in some samples but were estimated to be well below the FDA interim limits; the results are provided in Appendix B. Impurities that were below the detection or quantitation limit in some hand sanitizer samples were estimated as $\approx 0 \%$ (by mass) in Figure 11 and Appendix B.
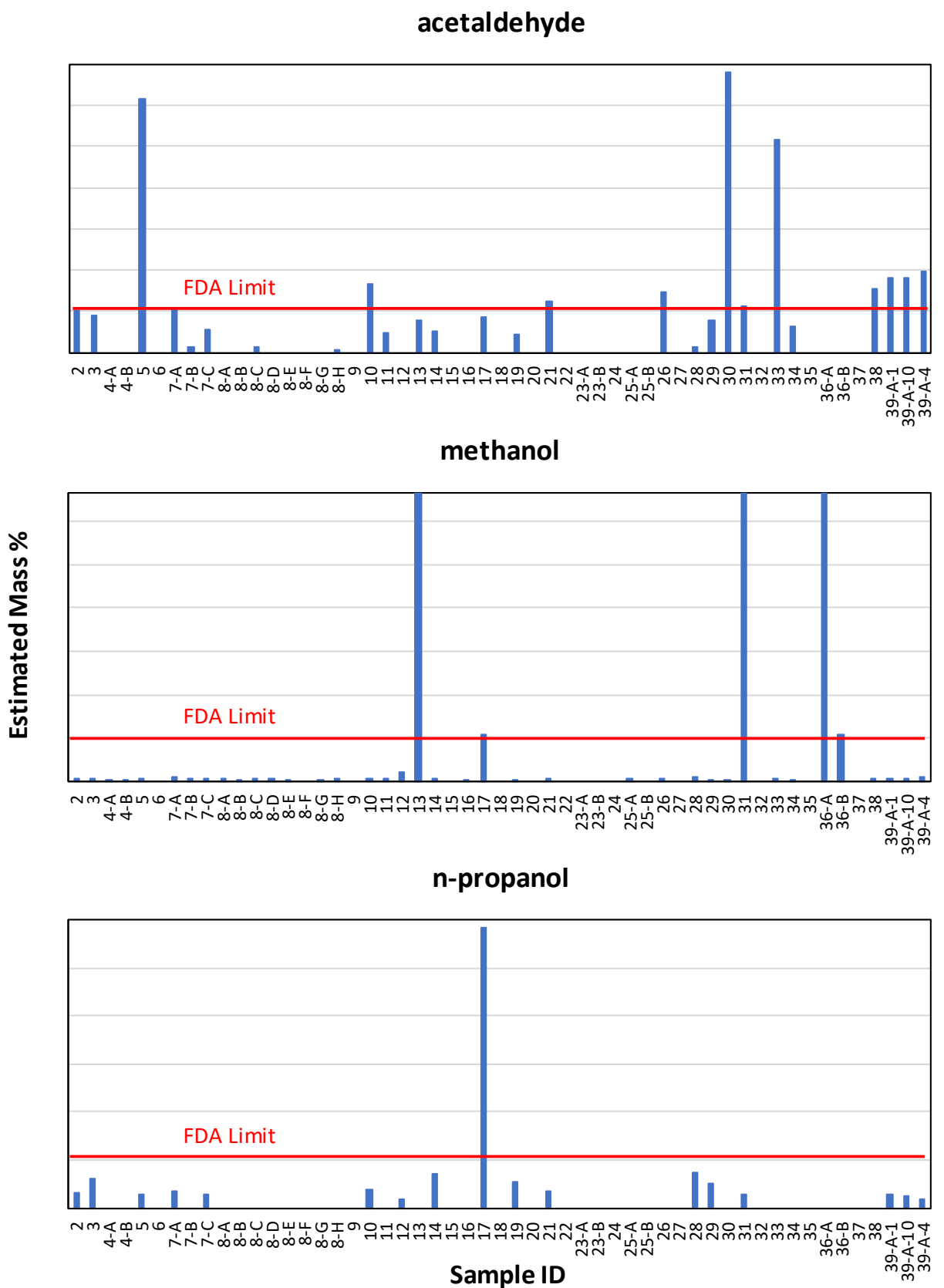

Figure 11. NMR estimates of impurities above the FDA limits in some hand sanitizers. 
The NMR methods presented here provide a straightforward means of quantifying ethanol and many impurities with regulated limits in hand sanitizer samples. At the outset of the study, potential interferences and impurities that might be observed were unknown and therefore a simple sample preparation procedure involving dilution in $\mathrm{D}_{2} \mathrm{O}$ was used for general screening purposes with no quantitative internal standard. Based on the collection of samples evaluated in this study, no sample interactions or overlapping signal interferences were observed for the internal standard dimethyl sulfone, suggesting it could be broadly applicable for qNMR purposes with hand sanitizer formulations. While detection and quantification limits were not specifically established in this work for NMR, they appear to be sufficient for the regulated levels for most components of interest with the exception of benzene and acetal. In addition, one could use an intrinsic component as an internal standard based on an independent estimate of that component, which was employed here in a retrospective analysis based on ethanol content estimated by the ATR-FTIR method. An external standard approach could also potentially be used but was not investigated

\subsubsection{GC Results}

GC is a high-resolution separation technique that, with an appropriate column, can separate many alcohols, solvents, and impurities in hand sanitizer formulations. FIDs are simple, robust, and sensitive detectors that have a large linear response range. FIDs are less expensive than mass spectrometers and are typically found in most analytical laboratories. Thus, GC-FID is a good candidate for the analysis of hand sanitizers. However, depending on the sample capacity of the column used, this technique often requires sample preparation and dilution.

In this work, the first samples analyzed by GC-FID were simple, non-gel hand sanitizers following the WHO/FDA recommended formulations. The addition of an internal standard and dilution were necessary for sample preparation of the simple formulations. Water was selected for this purpose because it is not detected by the FID and does not interfere with any other peaks. The samples were diluted 200-fold, and a split ratio of 100:1 was used because of the high alcohol content in the hand sanitizers.

Calibrants were prepared in a similar way to mimic the preparation steps for the samples. Sample chromatograms of simple, non-gel formulation hand sanitizers are shown in Figure 12. An RXI-1301 column was used because it could separate many alcohols, solvents, and impurities found in the sample matrix. There are other columns available that may also provide adequate separations such as DB-624 [10].

Two control samples were prepared to verify the accuracy of the quantitative GC-FID measurements. The first control was prepared using SRM 2899a Ethanol-Water Solution (Nominal Mass Fraction $25 \%$ ), which is a certified reference material with a certified value for ethanol in a water matrix. The percent recovery for this control sample was $100.6 \%$. In addition to this control, a hand sanitizer solution that was prepared in the laboratory using the WHO/FDA formulation with a known amount of ethanol added (one of the ATR-FTIR calibrants), was analyzed. The recovery for this second control was $99.9 \%$. The ethanol values of the controls determined by GC-FID are in good agreement with the certified or 
gravimetric value, indicating that this method is appropriate for quantifying ethanol in hand sanitizers that follow the FDA formulation.

The internal standard used in the early stages of this work was $n$-propanol, which was chosen from past methods and experience at NIST for quantifying ethanol in ethanol/water solutions. However, the internal standard was changed from $n$-propanol to acetonitrile in later analysis because $n$-propanol is a potential impurity in hand sanitizers (Table 1). It is not expected that the ethanol values were biased significantly for the analyses that used $n$-propanol as an internal standard because the estimated $n$-propanol content in the samples was typically low (below $300 \mu \mathrm{g} / \mathrm{g}$ ) whereas the $n$-propanol content of the internal standard spiked into the samples was high ( $73.6 \%$ by mass). Changing the internal standard from $n$-propanol to acetonitrile did not cause any apparent problems with the method.

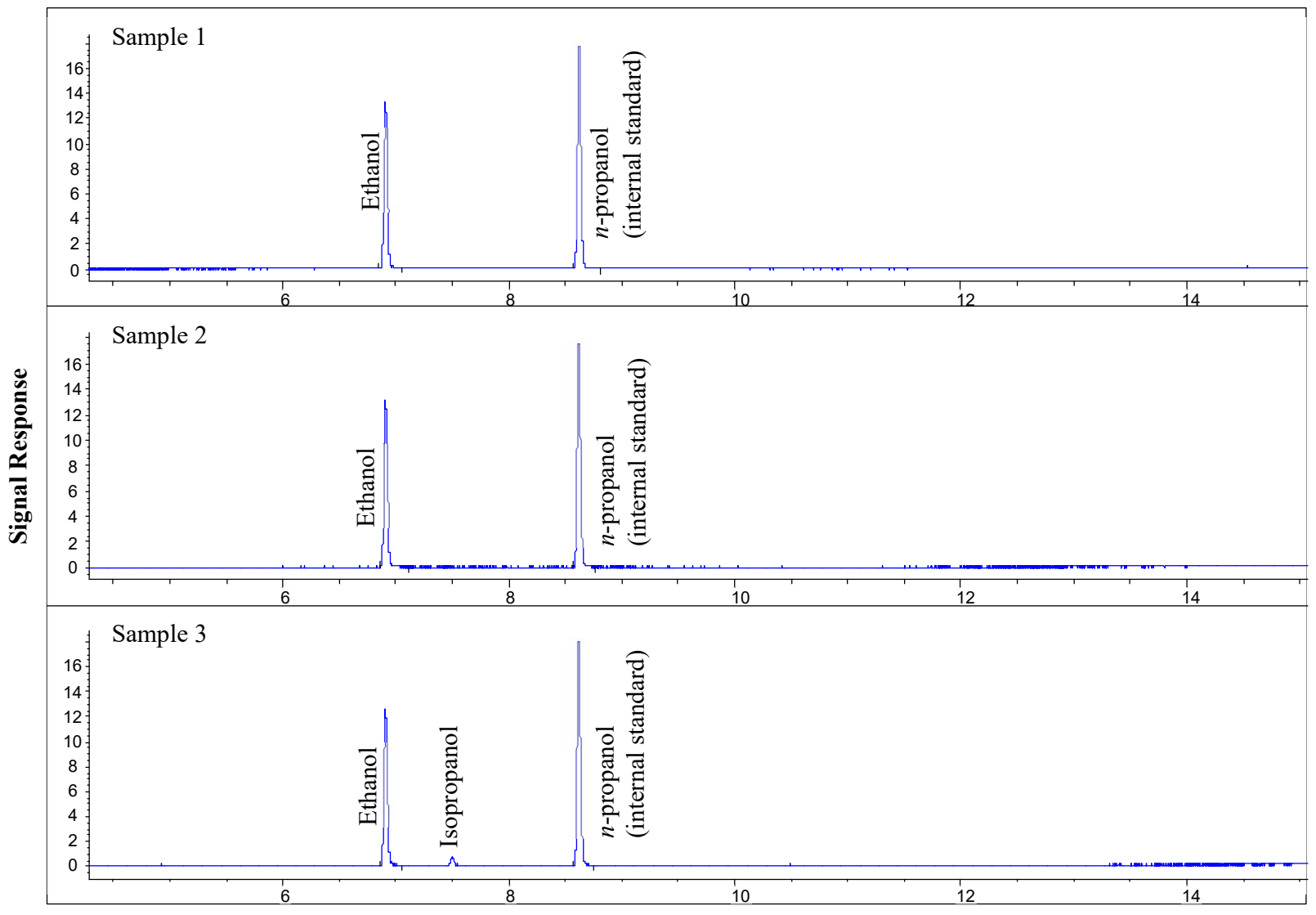

Retention Time (min)

Figure 12. Example GC-FID chromatograms of simple, non-gel formulation hand sanitizers. 
Samples with more advanced formulations, including gel hand sanitizers, can also be analyzed with this method after a sight modification of the sample preparation procedure by adding a small amount of $\mathrm{HCl}$ to make the sample less viscous before final dilution. The addition of the $\mathrm{HCl}$ or the more complex matrix of the gel hand sanitizers did not pose any interferences or issues during analysis. Figure 13 shows sample chromatograms of three gel hand sanitizer samples along with a calibrant.

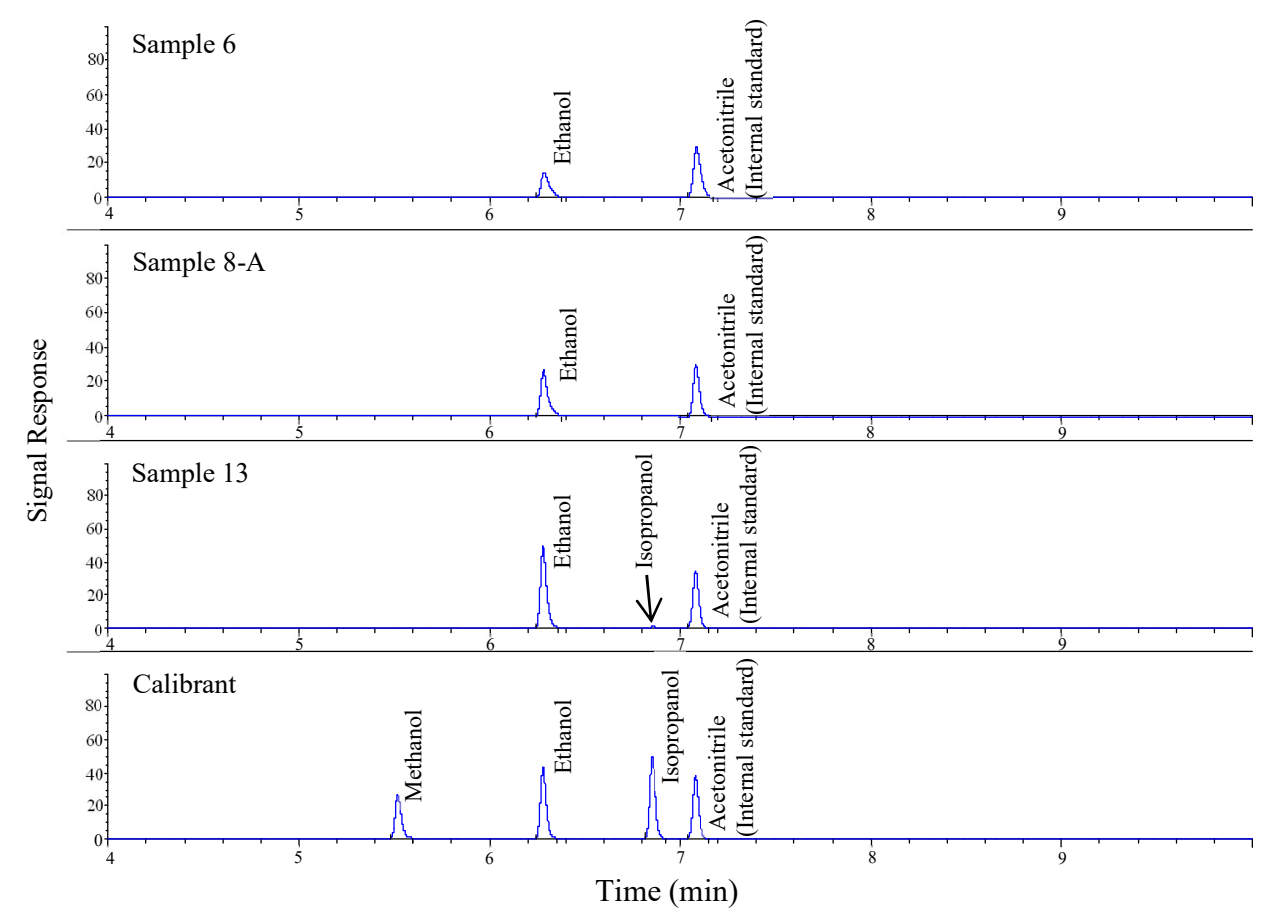

Figure 13. Example GC-FID chromatograms for more complex (gel) hand sanitizer formulations and a calibrant.

Even though many of the hand sanitizer formulations are complex with several ingredients including polymers and fragrances (see Appendix A), it is unlikely that the matrix would cause any issues with the quantification of alcohol by this method. The dilution of the samples with water does help eliminate matrix effects. However, this approach needs to be validated with matrix certified reference materials or through spiking experiments, which has not been performed in this study.

GC-FID was also used to identify and quantitate commonly found impurities in hand sanitizer formulations, including the impurities that the FDA has set interim limits (Table 1). Figure 14 shows samples chromatograms of impurities found in two hand sanitizer samples and a calibrant. 


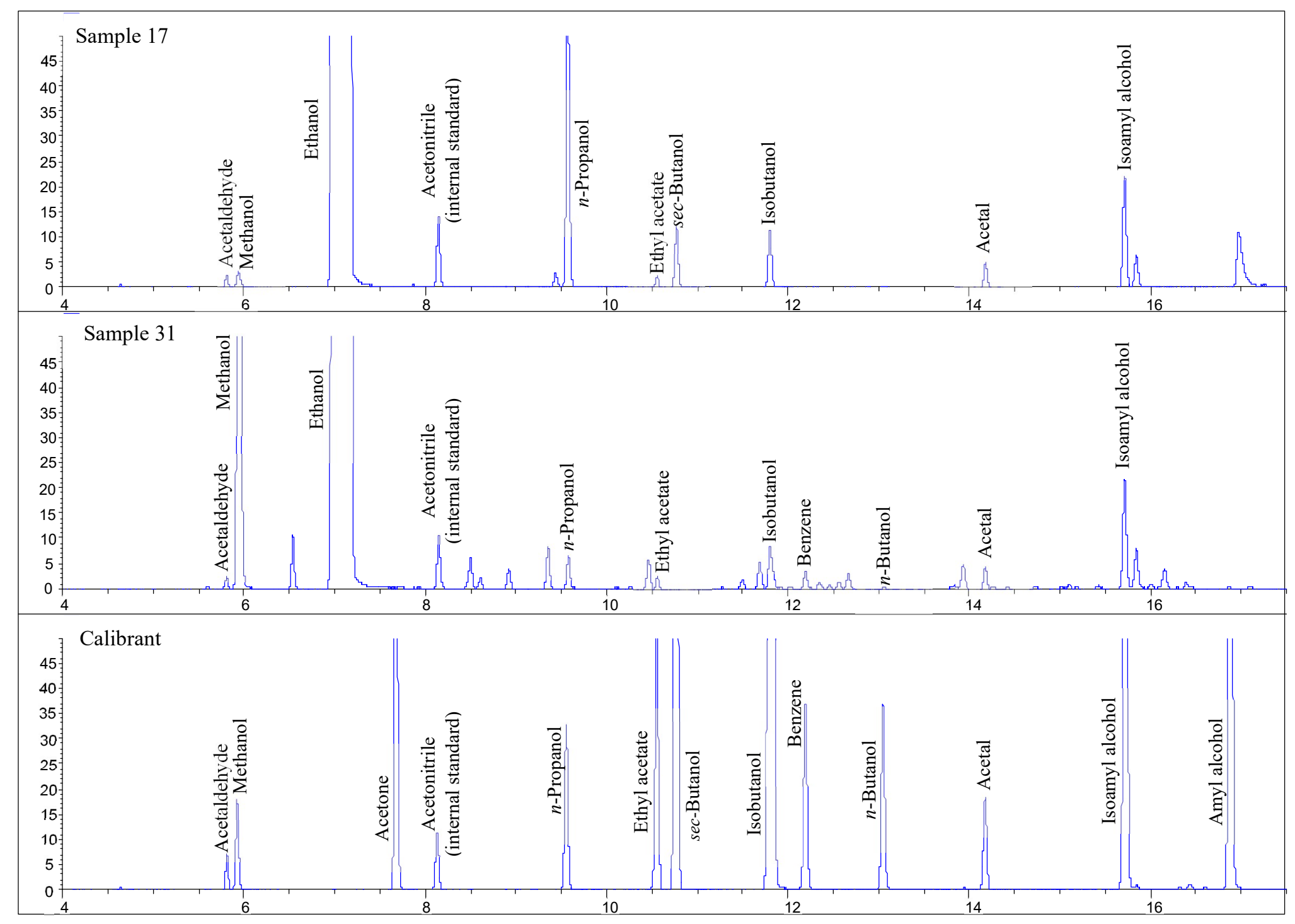

Figure 14. Example GC-FID chromatograms for impurities in two hand sanitizers and a calibrant. 
The results for impurities quantified by GC-FID for select hand sanitizer samples are summarized in Tables 9 and 10. The tables also note (with a checkmark) if the identity of the impurity was confirmed by NMR and/or GC-MS. Impurities that were found to be higher than the FDA interim levels in one or more samples are highlighted in Table 9, and impurities that were below the FDA interim levels in the select samples tested are listed in Table 10. Corresponding plots of the GC-FID results for the impurities above and below the FDA interim limits are presented in Figure 15 and Appendix C, respectively.

Five replicates portions of each Sample 39-A-1, Sample 33, and Sample 7-A were prepared to determine repeatability of the sample preparation protocol for impurity determination. The repeatability was good for most impurities with a \%RSD of less than $3 \%$ except for acetaldehyde and acetal. The \%RSD for acetaldehyde was $10 \%$ and $14 \%$ for Sample $39-\mathrm{A}-1$ and Sample 7-A, respectively. The \%RSD was higher for acetal with results of $23 \%$ and $38 \%$ for Sample 39-A-1 and Sample 7-A, respectively. Acetaldehyde may form acetal in the presence of ethanol. It is unclear if the preparation and dilution of samples could affect the equilibrium of this reaction, which might contribute to the increased variability in the results. It should be noted that in the USP monograph for determining impurities in alcohol, the sum of acetaldehyde and acetal is used to express the content of acetaldehyde in ethanol [8].

Calibrants were prepared in DMSO because all impurity standards were soluble in this solvent and it did not chromatographically interfere with the analytes. However, a high inlet temperature was used to ensure that the DMSO is vaporized in the injection port. Preparing calibrants containing multiple volatile analytes was a challenge. Lower volatility impurities were added first followed by the addition of the more volatile impurities to minimize evaporation. Because some calibrants were prepared from stock solutions that were subsequently diluted, any potential preparation errors would propagate to the lower level calibrants. To help identify and compensate for these potential issues, three independent stock solutions were prepared and appropriately diluted to yield solutions that were used to generate the calibration curve.

The FDA interim limit for benzene is $2 \mathrm{ppm}(\mu \mathrm{g} / \mathrm{g})$. Benzene was challenging to quantify at this low level using this GC-FID method. A calibrant was prepared at $1.6 \mu \mathrm{g} / \mathrm{g}$; however, the signal was very low and was not used in the calibration curve although a peak was detected at this level. Only benzene levels higher than the next calibration point of $16.6 \mu \mathrm{g} / \mathrm{g}$ were within the calibration range and could be properly quantified. Benzene was detected by GCFID in seven hand sanitizers (Sample 7-A, Sample 12, Sample 8-A, Sample 16, Sample 45, Sample 37, and Sample 31). Of these samples, the signal was within the calibration range only in Sample 31 (Table 9). The levels were too low to confirm by NMR, and only Sample 31 was confirmed by GC-MS in full scan mode. These samples were reanalyzed by GC-MS in selected ion mode $(\mathrm{m} / \mathrm{z} 78,77$, and 51$)$ to confirm the identity of benzene. All hand sanitizers that were identified by GC-FID to contain benzene were confirmed by GC-MS.

The hand sanitizer samples were not diluted for the analysis of impurities because most required a sensitive detection limit. The matrix of the hand sanitizer could pose a problem for quantitation, and more work using this method needs to be performed to check if there are matrix effects (e.g., overlapping matrix peaks). In addition, no certified reference materials were available to check the accuracy of the quantitation. 
Table 9. Quantitative GC-FID results for impurities over the FDA limits in select hand sanitizers.

\begin{tabular}{|c|c|c|c|c|c|c|c|c|c|c|c|c|c|}
\hline & \multicolumn{2}{|c|}{ Acetal } & \multicolumn{3}{|c|}{ Acetaldehyde } & \multicolumn{2}{|c|}{ Benzene } & \multicolumn{3}{|c|}{ Methanol } & \multicolumn{3}{|c|}{ n-Propanol } \\
\hline $\begin{array}{c}\text { Sample } \\
\text { ID }\end{array}$ & $\begin{array}{c}\mu \mathrm{g} / \mathrm{g} \\
(\mathrm{ppm})\end{array}$ & $\begin{array}{l}\text { GC- } \\
\text { MS }\end{array}$ & $\begin{array}{c}\mu \mathrm{g} / \mathrm{g} \\
(\mathrm{ppm})\end{array}$ & NMR & $\begin{array}{l}\text { GC- } \\
\text { MS }\end{array}$ & $\begin{array}{c}\mu \mathrm{g} / \mathrm{g} \\
(\mathrm{ppm})\end{array}$ & $\begin{array}{l}\text { GC- } \\
\text { MS }\end{array}$ & $\begin{array}{c}\mu \mathrm{g} / \mathrm{g} \\
(\mathrm{ppm})\end{array}$ & NMR & $\begin{array}{l}\text { GC- } \\
\text { MS }\end{array}$ & $\begin{array}{c}\mu \mathrm{g} / \mathrm{g} \\
(\mathrm{ppm})\end{array}$ & NMR & $\begin{array}{l}\text { GC- } \\
\text { MS }\end{array}$ \\
\hline 5 & $1740+$ & $\checkmark$ & $795+$ & $\checkmark$ & $\checkmark$ & ND & & 36.5 & $\checkmark$ & $\checkmark$ & 176 & $\checkmark$ & $\checkmark$ \\
\hline $7-\mathrm{A}$ & 118 & $\checkmark$ & 141 & $\checkmark$ & $\checkmark$ & $6.6 \ddagger$ & & 54.5 & $\checkmark$ & $\checkmark$ & 339 & $\checkmark$ & $\checkmark$ \\
\hline 8-A & $3.9 \ddagger$ & & $1.01 \ddagger$ & & & $3.5 \ddagger$ & & $17.2 \ddagger$ & $\checkmark$ & & $21.5 \ddagger$ & & \\
\hline 12 & ND & & $2.03 \ddagger$ & & & $2.9 \ddagger$ & & 113 & $\checkmark$ & $\checkmark$ & 195 & & $\checkmark$ \\
\hline 13 & 186 & $\checkmark$ & 56.9 & $\checkmark$ & $\checkmark$ & ND & & $7910 \dagger$ & $\checkmark$ & $\checkmark$ & 197 & & $\checkmark$ \\
\hline 16 & $5.4 \ddagger$ & & $3.13 \ddagger$ & & & $3.1 \neq$ & & $10.2 \ddagger$ & $\checkmark$ & & ND & & \\
\hline 17 & 213 & $\checkmark$ & 140 & $\checkmark$ & $\checkmark$ & ND & & 228 & $\checkmark$ & $\checkmark$ & $1878+$ & $\checkmark$ & $\checkmark$ \\
\hline 18 & $6.7 \ddagger$ & & $4.44 \ddagger$ & & & ND & & $5.4 \ddagger$ & & & $20.9 \ddagger$ & & \\
\hline 21 & 393 & $\checkmark$ & 136 & $\checkmark$ & $\checkmark$ & ND & & 35.8 & $\checkmark$ & $\checkmark$ & 524 & $\checkmark$ & $\checkmark$ \\
\hline $23-\mathrm{A}$ & $3.6 \ddagger$ & & $0.9 \ddagger$ & & & ND & & ND & & & $9.8 \ddagger$ & & \\
\hline 30 & 531 & $\checkmark$ & $760+$ & $\checkmark$ & $\checkmark$ & ND & & $19.5 \ddagger$ & $\checkmark$ & & 321 & & $\checkmark$ \\
\hline 31 & 167 & $\checkmark$ & 132 & $\checkmark$ & $\checkmark$ & 67.2 & $\checkmark$ & $8102+$ & $\checkmark$ & $\checkmark$ & 202 & $\checkmark$ & $\checkmark$ \\
\hline 32 & $10.3 \ddagger$ & & 9.3 & & & ND & & $4.2 \ddagger$ & & & $7.7 \ddagger$ & & \\
\hline 33 & 302 & $\checkmark$ & $625 t$ & $\checkmark$ & $\checkmark$ & ND & & $22.2 \ddagger$ & $\checkmark$ & $\checkmark$ & 353 & & $\checkmark$ \\
\hline 34 & 101 & $\checkmark$ & 67.1 & $\checkmark$ & $\checkmark$ & ND & & $14.1 \ddagger$ & $\checkmark$ & $\checkmark$ & ND & & \\
\hline $36-\mathrm{A}$ & ND & & $2.77 \ddagger$ & & & ND & & $145000+$ & $\checkmark$ & $\checkmark$ & $11.4 \ddagger$ & & \\
\hline $36-\mathrm{B}$ & ND & & $0.9 \ddagger$ & & & ND & & 719 & $\checkmark$ & $\checkmark$ & ND & & \\
\hline 37 & ND & & $0.1 \ddagger$ & & & $3.2 \ddagger$ & & ND & & & ND & & \\
\hline 39-A-1 & 183 & $\checkmark$ & 212 & $\checkmark$ & $\checkmark$ & ND & & 41.2 & $\checkmark$ & $\checkmark$ & 159 & $\checkmark$ & $\checkmark$ \\
\hline 45 & ND & & $3.20 \ddagger$ & & & $6.2 \ddagger$ & & $2.9 \ddagger$ & & & $5.1 \ddagger$ & & \\
\hline 46 & $9.9 \ddagger$ & & $6.18 \ddagger$ & & & ND & & $10.7 \ddagger$ & & $\checkmark$ & $9.2 \ddagger$ & & \\
\hline
\end{tabular}

$\dagger$ above highest calibration point; ¥ below lowest calibration point; ND not detected; $\checkmark$ identity was confirmed by NMR or GC-MS; Values in red are above the FDA interim limits. 
Table 10. Quantitative GC-FID results for impurities below the FDA limits in select hand sanitizers.

\begin{tabular}{|c|c|c|c|c|c|c|c|c|c|c|c|c|c|c|c|}
\hline & \multicolumn{3}{|c|}{ Acetone } & \multicolumn{3}{|c|}{ Ethyl acetate } & \multicolumn{3}{|c|}{ Isoamyl alcohol } & \multicolumn{3}{|c|}{ Isobutanol } & $n$-Butanol & \multicolumn{2}{|c|}{ sec-Butanol } \\
\hline $\begin{array}{l}\text { Sample } \\
\text { ID }\end{array}$ & $\begin{array}{c}\mu \mathrm{g} / \mathrm{g} \\
(\mathrm{ppm})\end{array}$ & NMR & GC-MS & $\begin{array}{c}\mu \mathrm{g} / \mathrm{g} \\
(\mathrm{ppm})\end{array}$ & NMR & GC-MS & $\begin{array}{c}\mu \mathrm{g} / \mathrm{g} \\
(\mathrm{ppm})\end{array}$ & NMR & GC-MS & $\begin{array}{c}\mu \mathrm{g} / \mathrm{g} \\
(\mathrm{ppm})\end{array}$ & NMR & GC-MS & $\begin{array}{c}\mu \mathrm{g} / \mathrm{g} \\
(\mathrm{ppm})\end{array}$ & $\begin{array}{c}\mu \mathrm{g} / \mathrm{g} \\
(\mathrm{ppm})\end{array}$ & GC-MS \\
\hline 5 & ND & $\checkmark$ & & 83.9 & $\checkmark$ & $\checkmark$ & 823 & $\checkmark$ & $\checkmark$ & $301 \ddagger$ & $\checkmark$ & $\checkmark$ & $14.7 \ddagger$ & ND & \\
\hline 8-A & ND & & & ND & $\checkmark$ & & ND & & & ND & & & ND & ND & \\
\hline 12 & ND & & & $34.0 \ddagger$ & $\checkmark$ & & ND & & & ND & & & ND & $15.7 \ddagger$ & \\
\hline 17 & ND & $\checkmark$ & & 123 & $\checkmark$ & $\checkmark$ & 662 & $\checkmark$ & $\checkmark$ & $357 \ddagger$ & $\checkmark$ & $\checkmark$ & $14.3 \ddagger$ & 403 & $\checkmark$ \\
\hline 18 & ND & & & ND & & & ND & & & ND & & & ND & ND & \\
\hline 21 & $5.6 \ddagger$ & $\checkmark$ & & 100 & $\checkmark$ & $\checkmark$ & $8.58 \ddagger$ & & & $164 \ddagger$ & $\checkmark$ & $\checkmark$ & ND & ND & \\
\hline $23-\mathrm{A}$ & ND & & & ND & & & ND & & & ND & & & ND & ND & \\
\hline 30 & $6.3 \ddagger$ & & & $61.1 \ddagger$ & $\checkmark$ & & 499 & & $\checkmark$ & $450 \ddagger$ & $\checkmark$ & $\checkmark$ & ND & ND & \\
\hline $36-\mathrm{A}$ & ND & & & $11.8 \ddagger$ & & & ND & & & ND & & & ND & ND & \\
\hline $36-\mathrm{B}$ & ND & & & $13.3 \ddagger$ & & & ND & & & ND & & & ND & ND & \\
\hline 37 & ND & & & ND & & & ND & & & ND & & & ND & ND & \\
\hline $39-A-1$ & ND & $\checkmark$ & & 62.7 & $\checkmark$ & $\checkmark$ & 774 & $\checkmark$ & $\checkmark$ & $281 \neq$ & $\checkmark$ & $\checkmark$ & 8 & ND & \\
\hline 45 & $8.8 \ddagger$ & & & $39.9 \ddagger$ & & & ND & & & ND & & & ND & ND & \\
\hline 46 & $40.8 \ddagger$ & & $\checkmark$ & $9.3 \ddagger$ & & & ND & & & ND & & & ND & ND & \\
\hline
\end{tabular}

¥ below lowest calibration point; ND not detected; $\checkmark$ identity was confirmed by NMR or GC-MS 


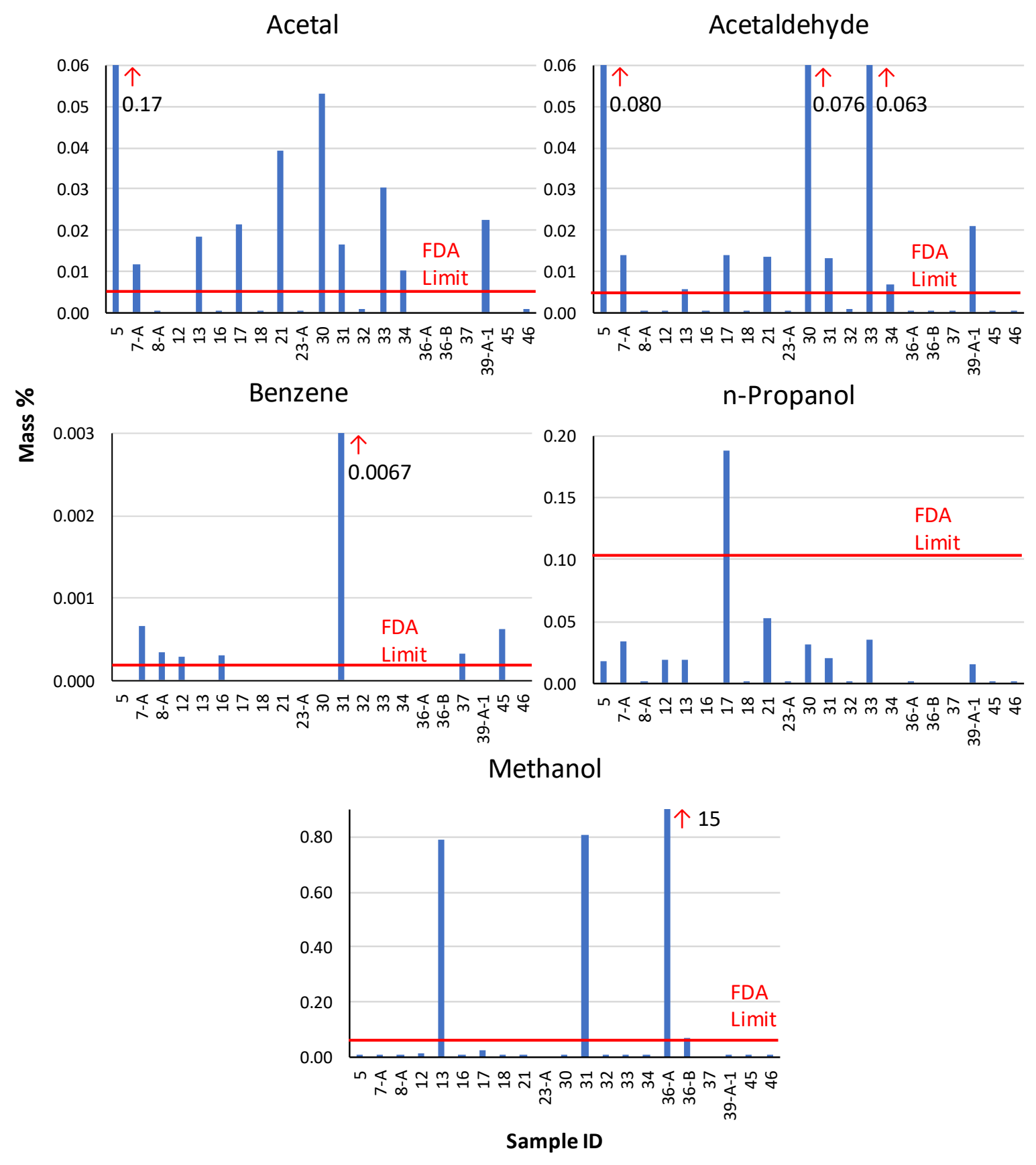

Figure 15. Plots of GC-FID results for impurities above FDA limits in some hand sanitizers. 
Impurity identification by GC-FID was primarily done by comparing the retention of the samples to that of the retention time of known compounds. GC-MS was used to provide additional confidence in the identification of impurities by comparing electron impact mass spectra with the NIST Mass Spectral Database.

The GC-FID method presented here provides a reliable and straight forward method for the quantitation of active ingredients and impurities in alcohol-based hand sanitizers. Most analytical laboratories have access to this technique, and columns are readily available for alcohol measurements. The relatively long chromatographic analysis time reported here can be time consuming for high throughput laboratories. However, total run times can be modified from the method described to decrease the total time by increasing the oven temperature ramp after the target analytes eluted. The major limitation of GC-FID is that separate sample preparations and methods are required to quantify ethanol and the impurities due to the large difference in content in hand sanitizer formulations. A method for determining alcohol and impurities in a single method using triple quadrupole mass spectrometry has been outlined by the FDA [10].

\subsubsection{LC-UV Results}

LC-UV was investigated because it can be used for measuring ethanol, methanol, and isopropanol in hand sanitizers either by direct injection or with minimal preparation of the samples. A conventional reverse-phase separation was used with a water/acetonitrile mobile phase that permitted detection at $200 \mathrm{~nm}$. A $5 \%$ acetonitrile/ $95 \%$ water (volume fractions) mobile phase provided baseline resolution of the three primary alcohols in a $10 \mathrm{~min}$ separation, as shown in the lower chromatogram for the alcohol mixture in Figure 16.

The shoulder on the chromatographic peak for methanol was always noted (Figure 16) and correlated with the area of the major peak as a function of methanol content. Thus, the area of the shoulder peak was included in the signal for methanol. The small peak at $\approx 4 \mathrm{~min}$ in the alcohol calibrant was a 'system' peak whose area did not correlate with the content of any of the alcohols. The chromatographic peak for ethanol displays 'peak fronting' in calibrants and samples with ethanol content $>\approx 60 \%$ (by volume) and was also prominent for gel hand sanitizer formulations.

The LC-UV method was successful for the analysis of non-gel sanitizer formulations, providing good overall agreement for ethanol determination compared with the other methods. However, there are several factors that potentially constrain the utility of LC-UV for alcohol determination in some hand sanitizer formulations, particularly for gel samples. The first is the relatively modest detectability of the alcohols at the lowest accessible UV wavelength, $200 \mathrm{~nm}$, which limits the detectability for alcohol determinations to values greater than approximately $1 \%$. Secondly, most organic compounds have some absorbance at $200 \mathrm{~nm}$, so the selectivity is low for determining the alcohols without potential interference from additional product ingredients or unwanted impurities. A third issue is the comparatively low retention of the alcohols, at least in the reverse-phase separation tested here. Low retention affords only limited flexibility to vary the mobile phase composition to enhance the chromatographic selectivity required for more complex samples. 


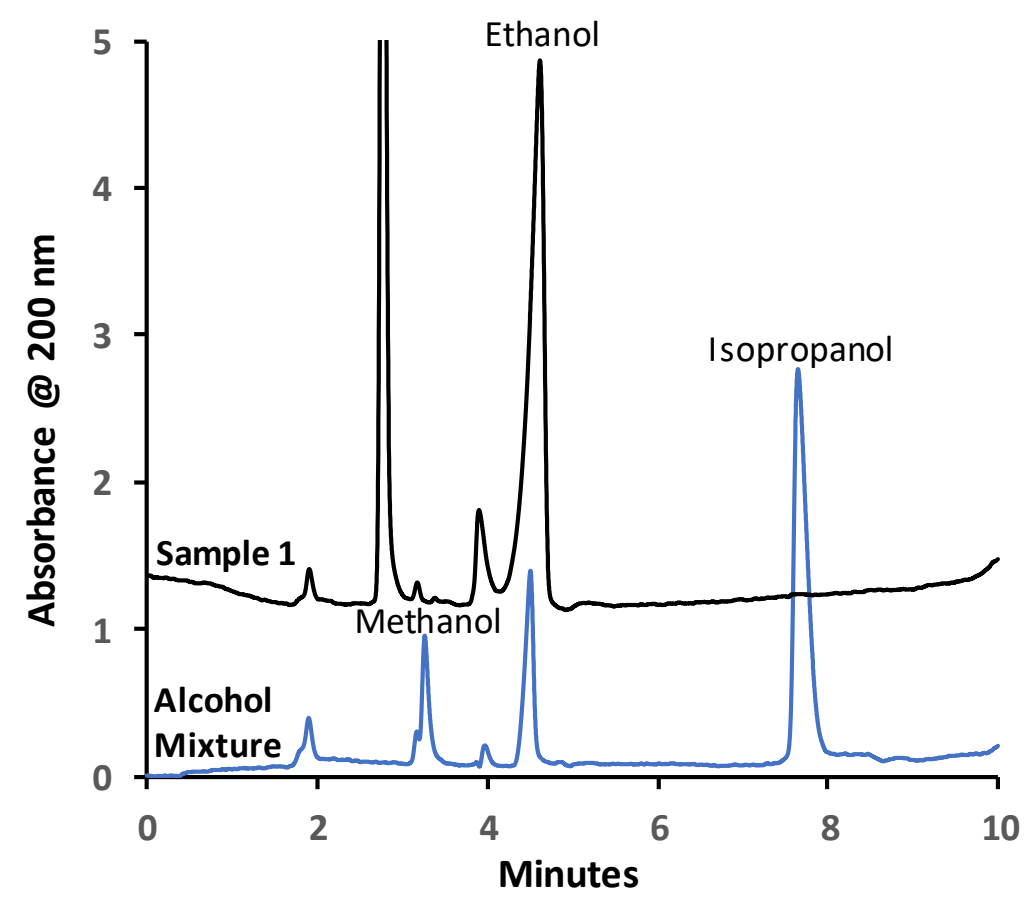

Figure 16. Separation and detection of a non-gel hand sanitizer (upper chromatogram) and an alcohol mixture (lower chromatogram) using LC-UV.

Gel hand sanitizers contain polymeric additives to enhance their viscosity. Sample preparation by the addition of $\mathrm{HCl}$ greatly lowered the viscosity of the sample, presumably by protonating the intermolecular hydrogen bonds of the gelling polymer. However, after addition of $\mathrm{HCl}$, the polymer is still present. Upon injection onto the $\mathrm{LC}$ column, precipitation of the polymer in the initial $5 \%$ acetonitrile mobile phase limited the suitable injection volume to $1 \mu \mathrm{L}$ and resulted in increased mobile phase back pressure as more samples were injected. To elute longer-retained sample components and minimize the effect of the effects of a gelling polymer, a flush cycle was introduced following the injection of each gel hand sanitizer sample. Although gradient elution solvent programs are possible, this adds greatly to the analysis time, requiring a significant delay time for the system to return to the initial conditions. Instead, after the analysis of each gel sample, a large injection volume of methanol, $100 \mu \mathrm{L}$, was used to elute longer retained components and minimize the impact of the accumulation of polymer on the column. A $100 \mu \mathrm{L}$ injection of acetonitrile seemed to be less effective at minimizing the increase in mobile phase back pressure attending the injection of gel samples. One gel hand sanitizer (Sample 36-A) still exhibited visible cloudiness after the addition of $\mathrm{HCl}$, and direct injection of this sample cause the column to become plugged. Two additional portions of Sample 36-A were analyzed, but they were filtered with a $0.2 \mu \mathrm{m}$ filter disk after $\mathrm{HCl}$ addition to alleviate the column plugging issue with this sample. However, in the absence of an internal standard to correct for sample evaporation, the measurement results for ethanol in these filtered samples were low relative to the other measurement techniques. 
In general, the results for ethanol in the gel hand sanitizers showed greater variability than the non-gel samples (see Table 6). This was likely to be caused by the effect of the polymer in the gel samples on the peak shape of the ethanol, where tailing was more pronounced in the LC determination. Thus, this LC-UV method was better suited for the determination of alcohols in non-gel hand sanitizers than for the gel formulations.

\subsubsection{Density Results}

To compare alcohol content in hand sanitizers reported in mass $\%$ and volume $\%$, the density of the hand sanitizers needed to be determined. Density measurements of liquids are typically straight forward; however, density determinations of finished gel hand sanitizers can be difficult due to high viscosity and entrapped air bubbles in some polymer-based formulations. Three methods were investigated in this study to measure the density of hand sanitizers: the Lang-Levy pipette method, the positive displacement pipette method, and the use of a hydrometer. It should be noted that other methods can be used to determine density that were not explored for this work (e.g., using a pycnometer).

The density values for 16 hand sanitizers using two to three different techniques are summarized in Table 11. The densities for the three techniques were obtained at different temperatures increasing the difficulty for density comparison. However, the difference in density values for the temperature range investigated should have minimal effect. For example, the density of a $60 \%$ ethanol in water solution (by volume) differs by only $0.6 \%$ over the experimental range of our measurements $\left(16^{\circ} \mathrm{C}\right.$ to $\left.23{ }^{\circ} \mathrm{C}\right)$ [13]. The densities of the three different methods were comparable except for a lower density value for the positivedisplacement pipette method for Sample 30.

The Lang-Levy method has previously been shown to be an accurate method for density determination, especially if sample volume is limited [15]. For non-gel hand sanitizer samples, this method was straightforward and easily implemented. However, this method was less suitable for viscous, gel samples due to the difficulty of drawing the viscous material into the pipette. Cleaning the Lang-Levy pipette after a viscous sample was introduced was also challenging. To measure the density of high viscosity samples by this method, the addition of a small amount of $\mathrm{HCl}$ was added to polymer-based samples $(0.5 \mu \mathrm{L}$ of $\mathrm{HCl}$ to $1 \mathrm{~mL}$ of sample) prior to measuring the density. It was assumed that the addition of $\mathrm{HCl}$ did not change the density of the sample. The advantage of this method is it only requires a small amount of sample. Disadvantages include the need of specialty Lang-Levy pipettes and the length of time that is required between measurements to clean and dry pipettes.

The use of a calibrated positive displacement pipette was the easiest method for gel samples and the most applicable to all hand sanitizers if appropriate precautions were taken. The result for the density of Sample 30 was lower using positive displacement pipette compared to the other methods. This was most likely due to the intentional removal of a small amount of sample inside the pipette tip when wiping off the excess sample from the outside of the pipette tip before transferring the sample to a pre-weighed receiving vial. It could also be due to a small entrapped air bubble of the sample or the incomplete transferring of the sample to 
the pre-weighed receiving vial. Because many of the gel samples contained bubbles, the small pipette volume for the positive displacement approach was used to sample the hand sanitizers in regions free of entrapped air, which is more challenging with the larger pipettes. Density measurements can be performed quickly with little sample using the positive displacement pipette method; however, care must be taken to ensure that the entire sample is transferred to the pre-weighed vial and that no excess sample is also transferred.

Table 11. Density results for select hand sanitizers obtained using three techniques

\begin{tabular}{|c|cc|cc|cc|}
\hline & \multicolumn{2}{|c|}{ Lang-Levy } & \multicolumn{2}{c|}{$\begin{array}{c}\text { Positive- } \\
\text { Displacement }\end{array}$} & \multicolumn{2}{c|}{ Hydrometer } \\
\hline Code & $\begin{array}{c}\text { Temp } \\
\left({ }^{\circ} \mathrm{C}\right)\end{array}$ & $\begin{array}{c}\text { Density } \\
(\mathrm{g} / \mathrm{mL})\end{array}$ & $\begin{array}{c}\text { Temp } \\
\left({ }^{\circ} \mathrm{C}\right)\end{array}$ & $\begin{array}{c}\text { Density } \\
(\mathrm{g} / \mathrm{mL})\end{array}$ & $\begin{array}{c}\text { Temp } \\
\left({ }^{\circ} \mathrm{C}\right)\end{array}$ & $\begin{array}{c}\text { Density } \\
(\mathrm{g} / \mathrm{mL})\end{array}$ \\
\hline 1 & 19.9 & 0.851 & 21.6 & 0.848 & 15.55 & \\
2 & 19.9 & 0.860 & 21.6 & 0.856 & 15.55 & \\
3 & 19.9 & 0.857 & 21.6 & 0.852 & 15.55 & \\
6 & 21.6 & 0.960 & 21.6 & 0.957 & 15.55 & 0.955 \\
$7-\mathrm{C}$ & 21.6 & 0.870 & 21.6 & 0.869 & 15.55 & 0.873 \\
$8-\mathrm{A}$ & 21.6 & 0.927 & 21.6 & 0.925 & 15.55 & 0.922 \\
$8-\mathrm{D}$ & 20.1 & 0.894 & 21.6 & 0.899 & 15.55 & \\
10 & 19.8 & 0.871 & 21.6 & 0.863 & 15.55 & \\
13 & 21.6 & 0.830 & 20.1 & 0.826 & 15.55 & 0.830 \\
17 & 21.6 & 0.849 & 21.6 & 0.849 & 15.55 & 0.851 \\
30 & 21.6 & 0.880 & 20.5 & 0.864 & 15.55 & 0.890 \\
$36-\mathrm{A}$ & 21.6 & 0.879 & 21.6 & 0.870 & 15.55 & 0.889 \\
$36-\mathrm{B}$ & 21.6 & 0.879 & 21.6 & 0.874 & 15.55 & 0.889 \\
$39-\mathrm{A}-1$ & 21.6 & 0.886 & 21.6 & 0.881 & 15.55 & 0.870 \\
40 & 19.8 & 0.855 & 21.6 & 0.845 & 15.55 & 0.850 \\
44 & 19.8 & 0.906 & 21.6 & 0.899 & 15.55 & 0.903 \\
\hline
\end{tabular}

* Specific gravity was converted to density using the density of water at $15.55{ }^{\circ} \mathrm{C}$ [16].

A hydrometer was investigated to measure the density (after converting specific gravity to density) of hand sanitizers because they are inexpensive, readily available, and generally advertised for use in determining ethanol content (typically in alcoholic beverages). The values obtained using the hydrometer for 11 select hand sanitizers were similar with the other techniques. However, the hydrometer method was challenging for the gel hand sanitizers. The hydrometer did not penetrate gel samples appropriately due to the high viscosity, which was alleviated by the addition of $\mathrm{HCl}$ to the sample. Other limitations of the hydrometer method are that large samples are required $(100 \mathrm{~mL})$, and the calibration temperature of $15.55^{\circ} \mathrm{C}$ is lower than the typical laboratory temperatures used for hand sanitizer analysis.

The positive displacement pipette method was selected to estimate the density of all hand sanitizers (results summarized in Appendix D) because of the applicability to both non-gel and gel samples and for the relative simplicity of the measurement approach. 
The primary use of the sample density in this work was to convert between mass \% and volume $\%$ for ethanol. However, another consideration is whether sample density can be used to reliably predict the ethanol content in hand sanitizers. The WHO/FDA

recommended hand sanitizer formulations are comprised primarily of water and ethanol with small amounts of glycerin and hydrogen peroxide. The simplicity of these formulations made them the most appropriate for evaluating whether ethanol content can be estimated based solely on the density. For this evaluation, the densities of the five calibrants prepared for the ATR-FTIR measurements using the WHO/FDA formulation were measured using the LangLevy technique and were used to prepare a calibration curve to correlate the amount of ethanol in the calibrant to the density. Then, the ethanol content for hand sanitizers that appeared to use the WHO/FDA formulation (based on labels and ingredient lists) were estimated from the measured sample density using the calibration curve of the standards. Table 12 shows the results of this determination comparing the estimated ethanol mass \% based on density to the measured values from the ATR-FTIR measurements. This is also shown graphically in Figure 17 along with an identity line where the predicted equals the measured volume $\%$ of ethanol. The estimated ethanol content of these samples based on density was within $5 \%$ of the measured ethanol content but still tended to be biased slightly high (i.e., most values were above the identity line). This model would only be applicable if the formulation was prepared to be very close in composition to the calibrants and if few impurities were present (for example, the same amount of glycerin was added to the hand sanitizer formulation and calibrants). To demonstrate this, the model was also applied to all hand sanitizers investigated in this study - both non-gel and gel formulations - and the results are shown graphically in Figure 18. This estimation approach based on density tends to overpredict the ethanol volume \% for many of the hand sanitizers and is not recommended for estimating ethanol content.

Table 12. Comparing the ethanol volume \% predicted from density to measured values by ATR-FTIR for hand sanitizers believed to follow the WHO/FDA guidance.

\begin{tabular}{|ccccc|}
\hline $\begin{array}{l}\text { Hand } \\
\text { Sanitizer ID }\end{array}$ & $\begin{array}{c}\text { Sample Density- } \\
\text { Measured (g/mL) }\end{array}$ & $\begin{array}{c}\text { Ethanol - Predicted } \\
\text { (Volume \%) }\end{array}$ & $\begin{array}{c}\text { Ethanol - Measured } \\
\text { (Volume \%) }\end{array}$ & \% Difference \\
\hline 1 & 0.851 & 83 & 81.8 & 1.9 \\
2 & 0.860 & 80 & 80.1 & -0.5 \\
3 & 0.857 & 81 & 77.4 & 4.6 \\
5 & 0.855 & 82 & 81.0 & 1.2 \\
10 & 0.871 & 76 & 77.2 & -2.2 \\
17 & 0.849 & 84 & 82.2 & 2.4 \\
19 & 0.860 & 80 & 77.7 & 2.8 \\
21 & 0.840 & 88 & 84.6 & 3.9 \\
40 & 0.855 & 82 & 80.9 & 1.0 \\
\hline
\end{tabular}




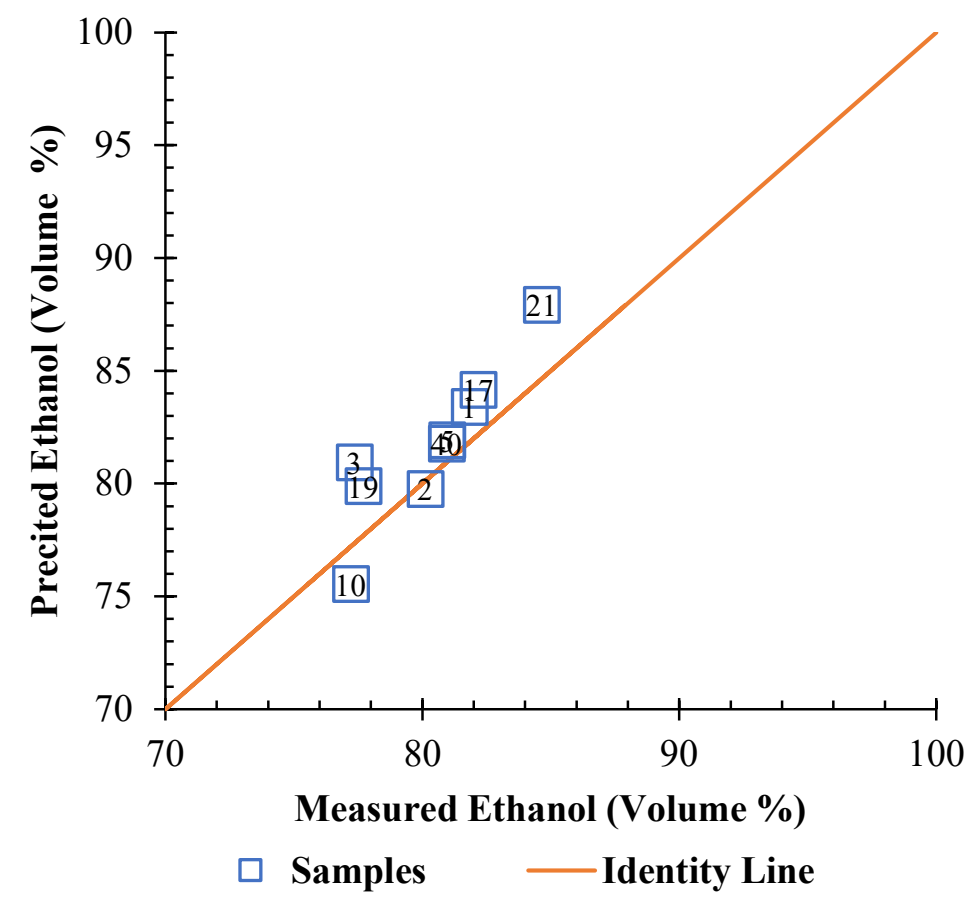

Figure 17. Plot of predicted (density) versus measured (ATR-FTIR) ethanol volume $\%$ results for hand sanitizers following the WHO/FDA formula.

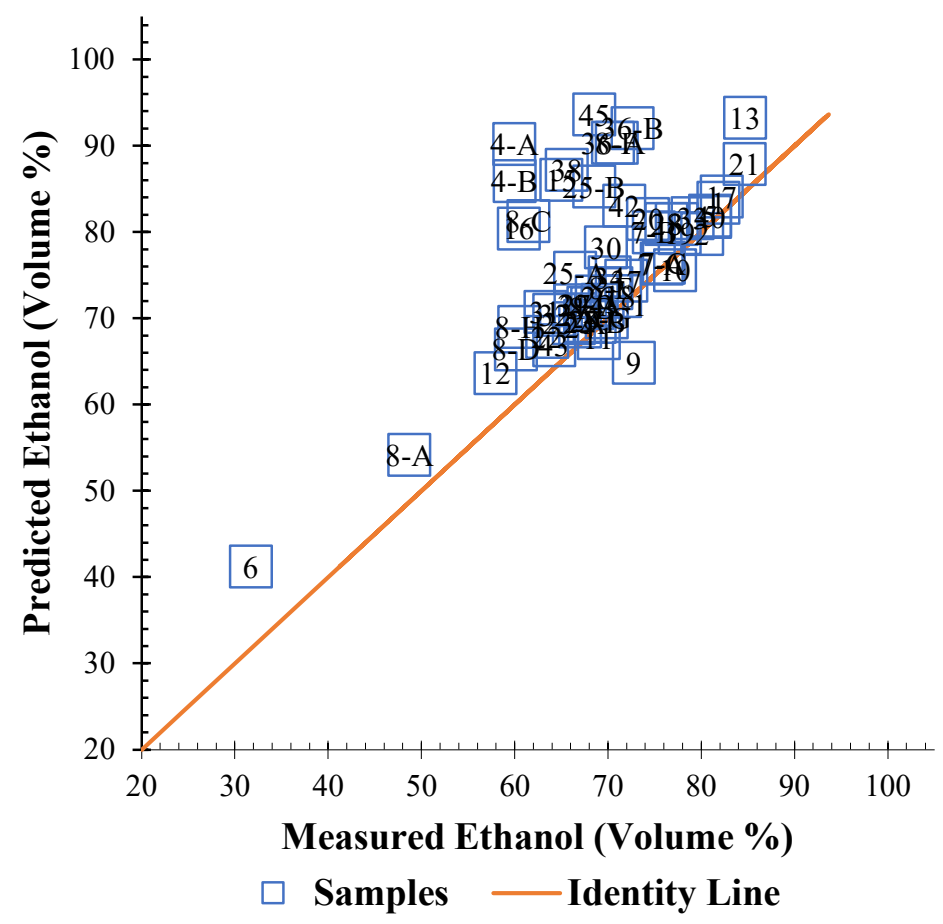

Figure 18. Plot of predicted (density) versus measured (ATR-FTIR) ethanol volume \% results for all hand sanitizers in this study. 


\section{Conclusions}

Four instrumental methods were evaluated for the analysis of hand sanitizers as part of this work including ATR-FTIR, NMR, GC-FID, and LC-UV. We evaluated 72 hand sanitizers that were obtained mostly from commercial sources. All samples were analyzed for ethanol content by ATR-FTIR, with a subset of 18 hand sanitizers selected for ethanol determinations by all methods. All methods can determine and provide comparable results for ethanol in both non-gel and gel hand sanitizer formulations. Based on the collective results in this work, it was estimated that all but five of the hand sanitizers analyzed contained ethanol at levels above the CDC recommended minimum of $60 \%$ (by volume). A caveat is that some of the samples that were slightly above the $60 \%$ (by volume) minimum based on single measurements by ATR-FTIR were not analyzed by additional methods for confirmation. The measurement of the sample density was also investigated as a simple approach to estimate the ethanol content in hand sanitizers, but for most samples it overpredicts the volume $\%$ of ethanol and was not deemed to be reliable.

The measurement of methanol and isopropanol are also of interest. Methanol is a harmful impurity that has been recently reported to exceed the FDA limits in many commercial hand sanitizers. Isopropanol is an approved alternative alcohol to ethanol in hand sanitizers as well as an allowable denaturant. All methods evaluated in this work can confirm the presence of methanol and isopropanol in hand sanitizers at percent levels, and quantitative methods for these alcohols were developed for NMR, GC-FID, and LC-UV. However, the LC-UV method was only able to quantitate these other alcohols when present at levels greater than approximately $1 \%$.

Two of the instrumental methods, qNMR and GC-FID, were also evaluated for the determination of impurity compounds, including methanol, at the much lower levels regulated by the FDA. Of these methods, NMR was able to determine most impurities at the requisite sensitivity levels set by the FDA limits, whereas GC-FID was able to determine all impurities. The applicability of each method for the analysis of hand sanitizers along with the strengths and weaknesses was described in detail in Section 3.3 and is summarized in Table 13.

Of the 72 hand sanitizers, 51 were screened for impurity content using NMR, and a subset of 21 samples were analyzed by GC-FID. The NMR results for isoamyl alcohol, isobutanol, $n$ - propanol, ethyl acetate, acetone, acetaldehyde, and methanol, relative to the FDA limit are plotted in Figure 19. In addition, the plot includes the GC-FID results relative to the FDA limits for acetal, benzene and acetaldehyde (in a few samples), which were found to be over the limit in some hand sanitizers but not quantifiable by NMR. In the plot, results that exceed the normalized limit (dashed black line) are represented with colored symbols, and results below the limit have black symbols as indicated in the legend. The plot further indicates the five hand sanitizers that were found to contain less than $60 \%$ ethanol (by volume; yellow boxes) based on the all-method average value. 
Table 13. Summary of method suitability for hand sanitizer measurements.

\begin{tabular}{|c|c|c|c|}
\hline METHOD & APPLICABILITY & STRENGTHS & WEAKNESSES \\
\hline GC-FID & $\begin{array}{l}\text { - Ethanol } \\
\text { - Isopropanol } \\
\text { - All regulated } \\
\text { impurities } \\
\text { - Non-gel and gel } \\
\text { samples }\end{array}$ & $\begin{array}{l}\text { - High selectivity } \\
\text { - High sensitivity } \\
\text { - High accuracy } \\
\text { - Detector response is very } \\
\text { stable } \\
\text { - Common method in } \\
\text { analytical labs } \\
\text { - Can measure all compounds } \\
\text { of interest }\end{array}$ & $\begin{array}{l}\text { - Each compound must be } \\
\text { calibrated separately } \\
\text { - Ethanol and impurities } \\
\text { require different } \\
\text { preparations }\end{array}$ \\
\hline NMR & $\begin{array}{l}\text { - Ethanol } \\
\text { - Isopropanol } \\
\text { - Most regulated } \\
\text { impurities } \\
\text { - Non-gel and gel } \\
\text { samples }\end{array}$ & $\begin{array}{l}\text { - Selective } \\
\text { - Sensitive for most impurities } \\
\text { - Requires no external } \\
\text { calibration } \\
\text { - Can measure most } \\
\text { compounds of interest in a } \\
\text { single, simple preparation } \\
\text { - High accuracy when used } \\
\text { with an internal standard }\end{array}$ & $\begin{array}{l}\text { - Sensitivity an issue for } \\
\text { low-level impurities } \\
\text { - Instrumentation } \\
\text { expensive } \\
\text { - Requires specific } \\
\text { expertise }\end{array}$ \\
\hline $\begin{array}{l}\text { ATR- } \\
\text { FTIR }\end{array}$ & $\begin{array}{l}\text { - Ethanol } \\
\text { - Identification of } \\
\text { other high-level } \\
\text { alcohols } \\
\text { - Non-gel and gel } \\
\text { samples }\end{array}$ & $\begin{array}{l}\text { - Simple, no sample } \\
\text { preparation requirements } \\
\text { - Rapid analysis } \\
\text { - Results for ethanol agree } \\
\text { with other methods }\end{array}$ & $\begin{array}{l}\text { - Primarily for ethanol } \\
\text { - Requires external } \\
\text { calibration }\end{array}$ \\
\hline LC-UV & $\begin{array}{l}\text { - Ethanol } \\
\text { - Methanol }>\approx 1 \% \\
\text { - Isopropanol > } \\
1 \% \\
\text { - Better for non-gel } \\
\text { samples }\end{array}$ & $\begin{array}{l}\text { - Relatively simple } \\
\text { - Direct analysis of samples } \\
\text { with little or no preparation } \\
\text { - Common method in } \\
\text { analytical labs }\end{array}$ & $\begin{array}{l}\text { - Selectivity limited } \\
\text { - Sensitivity limited } \\
\text { - Requires external } \\
\text { calibration }\end{array}$ \\
\hline
\end{tabular}

In total, 20 of the 51 hand sanitizers $(\approx 40 \%)$ were found to be over the limits for one or more impurity compounds. Acetaldehyde and/or acetal were the most frequently detected impurities and were over the limit in 14 samples $(\approx 30 \%)$, with one result (Sample 5 ) off the $y$-axis at 30 times the limit. Since the acetaldehyde and acetal content tended to correlate, the four hand sanitizers that have elevated acetaldehyde but show no value for acetal (Sample 2, Sample 10, Sample 26, and Sample 38) most likely contain acetal but were not measured by GC-FID. Six samples $(\approx 10 \%)$ were found to be over the limit for benzene, with one sample off the y-axis at 30 times the limit (Sample 31$)$. Four hand sanitizers $(\approx 8 \%)$ were found to be over the limit in methanol content, with one sample (Sample 36-A) off the y-axis at over 200 times the limit. Finally, one sample $(\approx 2 \%)$ was found to be over the limit for $n$-propanol (Sample 17). 


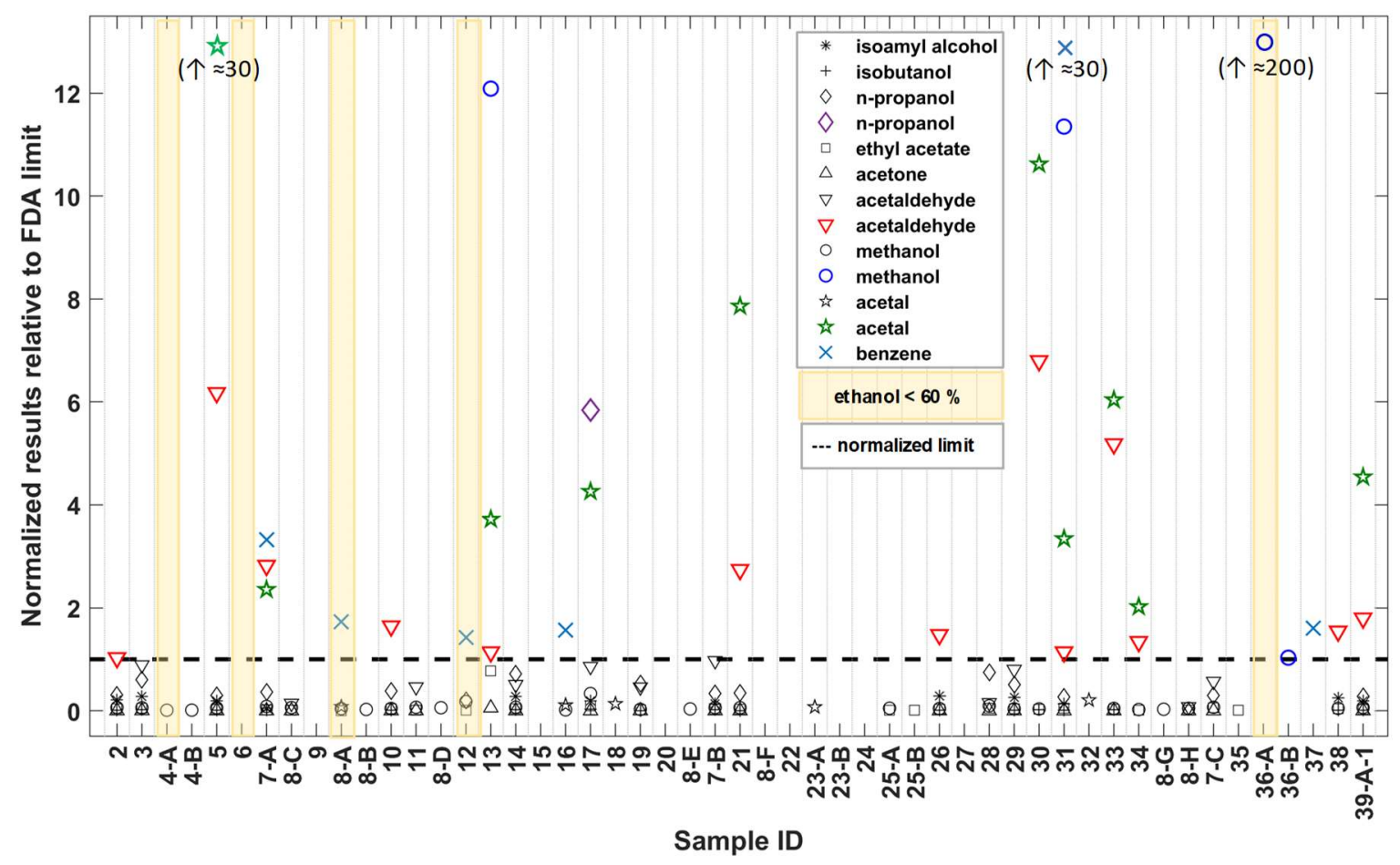

Figure 19. Summary of results for ethanol and impurities in most hand sanitizers evaluated in this work. 


\section{References}

[1] World Health Organization website (accessed January 2021), Timeline: WHO's COVID-19 Response (accessed January 2021).

https://www.who.int/emergencies/diseases/novel-coronavirus-2019/interactivetimeline\#!

[2] World Health Organization website (accessed January 2021). Coronavirus disease (COVID-19) overview. https://www.who.int/health-topics/coronavirus\#tab=tab_1

[3] Centers for Disease Control and Prevention website, How to Select and Use Hand Sanitizer (accessed January 2021). https://www.cdc.gov/coronavirus/2019ncov/prevent-getting-sick/hand-sanitizer.html

[4] U.S. Food and Drug Administration website (2020) Hand Sanitizers/COVID-19. https://www.fda.gov/drugs/coronavirus-covid-19-drugs/hand-sanitizers-covid-19

[5] U.S. Department of Health and Human Services, Food and Drug Administration, Center for Drug Evaluation and Research (2020) Temporary Policy for Preparation of Certain Alcohol-Based Hand Sanitizer Products During the Public Health Emergency (COVID-19) Guidance for Industry. https://www.fda.gov/media/136289/download

[6] U.S. Department of Health and Human Services, Food and Drug Administration, Center for Drug Evaluation and Research (2020) Policy for Temporary Compounding of Certain Alcohol-Based Hand Sanitizer Products During the Public Health Emergency in Effect Guidance for Industry. https://www.fda.gov/media/136118/download

[7] U.S. Department of Health and Human Services, Food and Drug Administration, Center for Drug Evaluation and Research (2020) Temporary Policy for Manufacture of Alcohol for Incorporation Into Alcohol-Based Hand Sanitizer Products During the Public Health Emergency (COVID-19) Guidance for Industry. https://www.fda.gov/media/136390/download

[8] United States Pharmacopeia (2020) Excerpted USP-NF and FCC Standards: A Hand Sanitizer Resource. https:/www.usp.org/sites/default/files/usp/document/healthquality-safety/usp-hand-sanitizer-ingredients.pdf

[9] U.S. Food and Drug Administration website (accessed January 2021), FDA updates on hand sanitizers consumers should not use. https:/www.fda.gov/drugs/drug-safety-andavailability/fda-updates-hand-sanitizers-consumers-should-not-use

[10] U.S. Food and Drug Administration (2020). Direct Injection Gas Chromatography Mass Spectrometry (GC-MS) Method for the Detection of Listed Impurities in Hand Sanitizers. https://www.fda.gov/media/141501/download

[11] World Health Organization (2010). Guide to Local Production: WHO-recommended Handrub Formulations. https://www.who.int/gpsc/5may/Guide_to_Local_Production.pdf

[12] https://www.canada.ca/en/revenue-agency/services/tax/technical-information/exciseact-2001-technical-information/canadian-alcoholometric-laboratory-table1996.html\#P31 2258

[13] OIML R 22, 1975 Edition, 1975 - International Alcoholometric Tables

[14] CRC Handbook of Chemistry and Physics. 100th Edition. CRC Press LLC.

[15] Sneigoski LT, Moody JR (1979) Determination of Serum and Blood Densities/ Analytical Chemistry 51: 1577-1578. https://doi.org/10.1021/ac50045a052

[16] Jones FJ, Harris GI (1992) Res. Natl. Inst. Stand. Technol. 97, 335. 
Appendix A: A summary of label information and product consistency for hand sanitizers analyzed.

\begin{tabular}{|c|c|c|c|c|}
\hline & & & & LABEL INFORMATION \\
\hline $\begin{array}{l}\text { Sample } \\
\text { ID }\end{array}$ & $\begin{array}{l}\text { Non- } \\
\text { gel or } \\
\text { Gel* }\end{array}$ & $\begin{array}{l}\text { Country } \\
\text { of } \\
\text { Origin }\end{array}$ & $\begin{array}{l}\text { Active } \\
\text { Ingredient }\end{array}$ & First Six (6) Inactive Ingredients \\
\hline 1 & $\begin{array}{c}\text { Non- } \\
\text { gel }\end{array}$ & N/A & $\mathbf{N} / \mathbf{A}$ & (Local Police Department Sample) \\
\hline 2 & $\begin{array}{l}\text { Non- } \\
\text { gel }\end{array}$ & N/A & N/A & (Local Police Department Sample) \\
\hline 3 & $\begin{array}{c}\text { Non- } \\
\text { gel }\end{array}$ & N/A & N/A & (Local Police Department Sample) \\
\hline 4-A & Gel & USA & $\begin{array}{l}\text { Ethyl } \\
\text { Alcohol 70\% } \\
\text { v/v }\end{array}$ & $\begin{array}{l}\text { DI Water, Glycerin, Carbomer 980, Benzophenone-4, Aloe } \\
\text { Vera, Tricaprylin }\end{array}$ \\
\hline 4-B & Gel & USA & $\begin{array}{l}\text { Ethyl } \\
\text { Alcohol 70\% } \\
\text { v/v }\end{array}$ & $\begin{array}{l}\text { Distilled Water, Glycerin, Carbomer 940, Benzophenone-4, } \\
\text { Aloe Vera, Aminomethyl Propanol }\end{array}$ \\
\hline 5 & $\begin{array}{l}\text { Non- } \\
\text { gel }\end{array}$ & USA & $\begin{array}{l}\text { Denatured } \\
\text { Alcohol } 80 \% \\
\text { v/v }\end{array}$ & Glycerin, Hydrogen Peroxide, Purified Water USP \\
\hline 6 & Gel & USA & $\begin{array}{l}\text { Ethyl } \\
\text { Alcohol 70\% } \\
\text { v/v } \\
\end{array}$ & $\begin{array}{l}\text { Water (Aqua), Glycerides Caprylate/Caprate, Aloe Vera Gel, } \\
\text { Isopropyl Myristate, Tocopherol Acetate, Acrylates/C10-C30 } \\
\text { Alkyl Acrylate Crosspolymer }\end{array}$ \\
\hline 7-A & Gel & USA & $\begin{array}{l}\text { Ethyl } \\
\text { Alcohol 70\% }\end{array}$ & $\begin{array}{l}\text { Water (Aqua), Isopropyl Alcohol, Propylene Glycol, } \\
\text { Glycerin, Tocopheryl Acetate, Acrylates/C10-30 Alkyl } \\
\text { Acrylate Crosspolymer }\end{array}$ \\
\hline 7-B & Gel & USA & $\begin{array}{l}\text { Ethyl Alcohol } \\
70 \%\end{array}$ & $\begin{array}{l}\text { Water (aqua), Isopropyl Alcohol, Propylene Glycol, Glycerin, } \\
\text { Tocopheryl Acetate, Acrylates/C10-C30 Alkyl Acrylated } \\
\text { Crosspolymer }\end{array}$ \\
\hline 7-C & Gel & USA & $\begin{array}{l}\text { Ethyl } \\
\text { Alcohol 70\% }\end{array}$ & $\begin{array}{l}\text { Water (aqua), Isopropyl Alcohol, Propylene Glycol, Glycerin, } \\
\text { Tocopheryl Acetate, Acrylates/C10-30 Alkyl Acrylate } \\
\text { Crosspolymer }\end{array}$ \\
\hline 8-A & Gel & N/A & $\begin{array}{l}\text { Ethyl } \\
\text { Alcohol 65\% }\end{array}$ & $\begin{array}{l}\text { Water, Aloe Barbadensis Leaf Extract, Acrylates/C10-30 Alyl } \\
\text { Acrylate Crosspolymer, Alcohol, Tetrahydroxypropyl } \\
\text { Ethylenediamine }\end{array}$ \\
\hline $8-\mathrm{B}$ & Gel & $\mathrm{N} / \mathrm{A}$ & $\begin{array}{l}\text { Ethyl Alcohol } \\
65 \%\end{array}$ & Water, Carbomer, Triethanolamine \\
\hline $8-\mathrm{C}$ & Gel & $\mathrm{N} / \mathrm{A}$ & $\begin{array}{l}\text { Ethyl Alcohol } \\
65 \%\end{array}$ & Water, Carbomer 940, Triethanolamine, Glycerin \\
\hline 8 -D & Gel & $\mathrm{N} / \mathrm{A}$ & $\begin{array}{l}\text { Ethyl Alcohol } \\
65 \%\end{array}$ & Water (Aqua), Carbomer 940, Triethanolamine \\
\hline $8-\mathrm{E}$ & Gel & China & $\begin{array}{l}\text { Ethyl Alcohol } \\
62.5 \%\end{array}$ & $\begin{array}{l}\text { Water, Carbomer 940, Triethanolamine, Glycerin, Aloe } \\
\text { Barbadensis Leaf Juice }\end{array}$ \\
\hline $8-\mathrm{F}$ & Gel & $\mathrm{N} / \mathrm{A}$ & $\begin{array}{l}\text { Ethyl Alcohol } \\
62.5 \% \\
\end{array}$ & $\begin{array}{l}\text { Denatured Dehydrated Alcohol 40B, Water, Aloe Barbadensis } \\
\text { Leaf Juice, Glycerin, Propylene Glycol, Carbomer }\end{array}$ \\
\hline $8-\mathrm{G}$ & Gel & China & $\begin{array}{l}\text { Ethyl Alcohol } \\
62.5 \%\end{array}$ & $\begin{array}{l}\text { Water (Aqua), Carbomer 940, Triethanolamine, Glycerin, Aloe } \\
\text { Barbadensis Leaf Juice }\end{array}$ \\
\hline
\end{tabular}




\begin{tabular}{|c|c|c|c|c|}
\hline $8-\mathrm{H}$ & Gel & N/A & $\begin{array}{l}\text { Ethyl Alcohol } \\
65 \%\end{array}$ & Water (Aqua), Carbomer, Triethanolamine \\
\hline 9 & Gel & Korea & Alcohol 62\% & $\begin{array}{l}\text { Water, Glycerin, Aloe Barbadensis Leaf Water ( } 9 \mathrm{ppm}) \text {, } \\
\text { Hamamelis Virginiana (Witch Hazel) Water, Sodium } \\
\text { Hyaluronate, Trehalose }\end{array}$ \\
\hline 10 & $\begin{array}{l}\text { Non- } \\
\text { gel }\end{array}$ & USA & $\begin{array}{l}\text { Ethyl } \\
\text { Alcohol 80\% } \\
\text { v/v }\end{array}$ & Glycerin, Hydrogen Peroxide, Purified Water USP \\
\hline 11 & Gel & USA & $\begin{array}{l}\text { Ethyl Alcohol } \\
62 \% \text { by } \\
\text { weight }\end{array}$ & $\begin{array}{l}\text { Water (Aqua), Glycerin, Tocopheryl Acetate, Isopropyl } \\
\text { Palmitate, Acrylates Coppolymer, Aminomethyl Propanol }\end{array}$ \\
\hline 12 & Gel & China & $75 \%$ Ethanol & $\mathbf{N} / \mathbf{A}$ \\
\hline 13 & $\begin{array}{c}\text { Non- } \\
\text { gel }\end{array}$ & $\mathbf{N} / \mathbf{A}$ & $\begin{array}{l}\text { Alcohol 80\% } \\
\text { v/v }\end{array}$ & Glycerin, Hydrogen Peroxide, Purified Water USP \\
\hline 14 & Gel & USA & $\begin{array}{l}\text { Ethyl Alcohol } \\
70 \% \mathrm{v} / \mathrm{v}\end{array}$ & $\begin{array}{l}\text { Aqua, Isopropyl Alcohol, Polysorbate 20, Carbomer, } \\
\text { Triethanolamine, Glycerin }\end{array}$ \\
\hline 15 & Gel & USA & $\begin{array}{l}\text { Ethyl Alcohol } \\
62 \% \mathrm{v} / \mathrm{v}\end{array}$ & $\begin{array}{l}\text { Deionized Water, Glycerin, Isopropyl Myristate, Aloe } \\
\text { Barbadensis Leaf Juice, Tocopheryl Acetate (Vitamin E), } \\
\text { Crosspolymer }\end{array}$ \\
\hline 16 & Gel & Mexico & $\begin{array}{l}\text { Ethyl } \\
\text { Alcohol 70\% } \\
\text { v/v }\end{array}$ & $\begin{array}{l}\text { Demineralized Water, Glycerin, Carbomer, Triethanolamine, } \\
\text { dmdm hydantoin, Aloe Barbadensis Extract }\end{array}$ \\
\hline 17 & $\begin{array}{c}\text { Non- } \\
\text { gel }\end{array}$ & $\mathbf{N} / \mathbf{A}$ & $\begin{array}{l}\text { Alcohol 80\% } \\
\text { v/v }\end{array}$ & Glycerin, Hydrogen Peroxide, Purified Water USP \\
\hline 18 & Gel & Canada & $\begin{array}{l}\text { Ethyl } \\
\text { Alcohol 62\% } \\
\text { v/v } \\
\text { (Technical } \\
\text { Grade) }\end{array}$ & $\begin{array}{l}\text { Water, Isopropyl Alcohol, Glcyerin, Carbomer, Aminomethyl } \\
\text { Propanol, Fragrance }\end{array}$ \\
\hline 19 & $\begin{array}{c}\text { Non- } \\
\text { gel }\end{array}$ & USA & $\begin{array}{l}\text { Ethyl alcohol } \\
70 \% \mathrm{v} / \mathrm{v}\end{array}$ & Water, Isopropyl alcohol, glycerin, Aloe Barbadensis Leaf Juice \\
\hline 20 & Gel & USA & $\begin{array}{l}\text { Ethyl } \\
\text { Alcohol 70\% }\end{array}$ & $\begin{array}{l}\text { Water (Aqua), Isopropyl Alcohol, Orange oil, Acrylates/C10- } \\
\text { C30 Alkyl Acrylate Crosspolymer, Glycerin, Aloe } \\
\text { Barbadensis Leaf Juice }\end{array}$ \\
\hline 21 & $\begin{array}{c}\text { Non- } \\
\text { gel }\end{array}$ & USA & $\begin{array}{l}\text { Ethyl } \\
\text { Alcohol 75\% }\end{array}$ & Purified Water USP, Glycerin, Hydrogen Peroxide \\
\hline 22 & $\begin{array}{l}\text { Non- } \\
\text { gel }\end{array}$ & N/A & $\begin{array}{l}\text { Ethyl Alcohol } \\
62 \%\end{array}$ & $\begin{array}{l}\text { Aloe Barbadensis Leaf Juice, Calendula Officinalis Flower } \\
\text { Extract, Chamomilla Recutita (Matricaria) Flower Extract, } \\
\text { Glycerin, Water }\end{array}$ \\
\hline 23-A & $\begin{array}{l}\text { Non- } \\
\text { gel }\end{array}$ & $\mathbf{N} / \mathbf{A}$ & $\begin{array}{l}\text { Ethyl } \\
\text { Alcohol 62\% }\end{array}$ & $\begin{array}{l}\text { Aloe Barbadensis Leaf Juice, Calendula Officinalis Flower } \\
\text { Extract, Chamomilla Recutita (Matricaria) Flower Extract, } \\
\text { Glycerin, Water, }\end{array}$ \\
\hline $23-\mathrm{B}$ & Gel & $\mathrm{N} / \mathrm{A}$ & $\begin{array}{l}\text { Ethyl Alcohol } \\
62 \%\end{array}$ & $\begin{array}{l}\text { Aloe Barbadensis Leaf Juice, Calendula Officinalis Flower } \\
\text { Extract, Chamomilla Recutita (Matricaria) Flower Extract, Citric } \\
\text { Acid, Glycerin, Hydroxypropyl Guar }\end{array}$ \\
\hline 24 & $\begin{array}{c}\text { Non- } \\
\text { gel }\end{array}$ & USA & $\begin{array}{l}\text { Ethyl alcohol, } \\
65 \% \mathrm{v} / \mathrm{v}\end{array}$ & $\begin{array}{l}\text { Purified Water USP, Glycerin USP, Lavender oil, Safflower Oil, } \\
\text { Almond Oil, Coconut Oil }\end{array}$ \\
\hline $25-\mathrm{A}$ & Gel & China & $\begin{array}{l}\text { Ethyl Alcohol } \\
62 \%\end{array}$ & $\begin{array}{l}\text { Water (Aqua), Isopropyl Alcohol, Glycerin, Carbomer, } \\
\text { Aminomethyl Propanol, Parfum }\end{array}$ \\
\hline $25-\mathrm{B}$ & Gel & China & $\begin{array}{l}\text { Ethyl Alcohol } \\
62 \%\end{array}$ & $\begin{array}{l}\text { Water (Aqua), Aloe Barbadensis Leaf Extract, Carbomer, } \\
\text { Parfum, Glycerin, Propylene Glycol }\end{array}$ \\
\hline
\end{tabular}




\begin{tabular}{|c|c|c|c|c|}
\hline 26 & Gel & USA & $\begin{array}{l}\text { ethyl alcohol } \\
62.5 \%\end{array}$ & $\begin{array}{l}\text { Purified Water, Carbomer, Aminomethylpropanol, Fragrance, } \\
\text { Glycerin, Propylene Glycol }\end{array}$ \\
\hline 27 & Gel & China & $\begin{array}{l}\text { Ethyl Alcohol } \\
75 \% \mathrm{v} / \mathrm{v}\end{array}$ & $\begin{array}{l}\text { Water, Triethanolamine, Carbomer, Aloe Barbadensis (Aloe } \\
\text { Vera) Gel, Fragrance, Glycerin }\end{array}$ \\
\hline 28 & Gel & USA & $\begin{array}{l}\text { Ethyl alcohol } \\
72 \%\end{array}$ & $\begin{array}{l}\text { Water (Aqua), Carbomer, Aminomethyl Propanol, Butylene } \\
\text { Glycol, Glycerin, Aloe Barbadensis Leaf Juice }\end{array}$ \\
\hline 29 & Gel & Canada & $\begin{array}{l}\text { Ethyl Alcohol } \\
70 \% \mathrm{v} / \mathrm{v}\end{array}$ & $\begin{array}{l}\text { Aminomethyl Propanol, Carbomer, Glycerin, Tocopheryl } \\
\text { Acetate, Water, }\end{array}$ \\
\hline 30 & Gel & USA & $\begin{array}{l}\text { Ethyl } \\
\text { Alcohol 62\% }\end{array}$ & $\begin{array}{l}\text { Purified Water (Aqua), Carbomer, Aminomethyl Propanol, } \\
\text { Cocos Nucifera (coconut) Fruit Extract, Lippia Citriodora } \\
\text { (Lemon Verbena) Leaf Extract, Aloe Barbadensis (Aloe } \\
\text { Vera) Leaf Juice }\end{array}$ \\
\hline 31 & Gel & Mexico & $\begin{array}{l}\text { Ethyl alcohol } \\
\mathbf{7 0 \%} \text { v/v }\end{array}$ & $\begin{array}{l}\text { Water (Aqua), Carbomer, Triethanolamine, Glycerin, } \\
\text { Propylene Glycol, Aloe Barbadensis Leaf Juice }\end{array}$ \\
\hline 32 & Gel & $\mathbf{N} / \mathbf{A}$ & $\begin{array}{l}\text { Ethyl } \\
\text { Alcohol } \\
62.5 \% \mathrm{v} / \mathrm{v}\end{array}$ & $\begin{array}{l}\text { Water (Aqua), Propylene Glycol, Dimethicone Peg-7 } \\
\text { Isosterate, Acrylates/C10-C30 Alkyl Acrylate Crosspolymer, } \\
\text { Aminomethylpropanediol, Tocopherol Acetate }\end{array}$ \\
\hline 33 & Gel & USA & $\begin{array}{l}\text { Ethyl } \\
\text { Alcohol } 70 \\
\% \text { v/v }\end{array}$ & Deionized Water, Glycerin, Carbomer, Amino Propanol \\
\hline 34 & Gel & USA & $\begin{array}{l}\text { Ethyl } \\
\text { Alcohol } \\
63.5 \% \\
\end{array}$ & Aminomethyl Propanol, Carbomer, Water \\
\hline 35 & Gel & N/A & N/A & (Dispenser station at NIST) \\
\hline 36-A & Gel & Mexico & $\begin{array}{l}\text { Ethyl } \\
\text { Alcohol 70\% } \\
\end{array}$ & Water, Carbomer, Triethanolamine, Glycerine \\
\hline 36-B & Gel & Mexico & $\begin{array}{l}\text { Ethyl } \\
\text { Alcohol 70\% } \\
\end{array}$ & Water, Carbomer, Triethanolamine, Glycerine \\
\hline 37 & Gel & China & $\begin{array}{l}\text { Ethyl } \\
\text { Alcohol 75\% } \\
\text { (v/v) }\end{array}$ & Water, Glycerin, Propylene Glycol, Carbomer \\
\hline 38 & Gel & USA & $\begin{array}{l}\text { Ethyl Alcohol } \\
62 \%\end{array}$ & $\begin{array}{l}\text { Water, Isopropyl Alcohol, Glycerin, Carbomer, Aminomethyl } \\
\text { Propanol, Fragrance }\end{array}$ \\
\hline $\begin{array}{c}39-A-1 \\
\text { to } 39- \\
\text { A-12 }\end{array}$ & Gel & Mexico & $\begin{array}{l}\text { Ethyl alcohol } \\
70 \%\end{array}$ & Water, Propylene Glycol, Carbomer, Sodium Hydroxide \\
\hline 40 & $\begin{array}{c}\text { Non- } \\
\text { gel }\end{array}$ & USA & $\begin{array}{l}\text { Alcohol 80\% } \\
\text { v/v }\end{array}$ & Glycerin, Hydrogen Peroxide, Purified Water USP \\
\hline 41 & Gel & China & $\begin{array}{l}\text { Ethyl Alcohol } \\
75 \% \mathrm{v} / \mathrm{v}\end{array}$ & $\begin{array}{l}\text { Water, Glycerin, Propylene Glycol, Carbomer, Triethanolamine, } \\
\text { Fragrance }\end{array}$ \\
\hline 42 & Gel & N/A & $\begin{array}{l}\text { Ethyl Alcohol } \\
65 \%\end{array}$ & $\begin{array}{l}\text { Water, Glycerin, Isopropyl Alcohol, Butylene Glycol, Carbomer, } \\
\text { Aminomethyl Propanol }\end{array}$ \\
\hline 43 & Gel & USA & $\begin{array}{l}\text { Ethyl Alcohol } \\
63 \% \mathrm{v} / \mathrm{v}\end{array}$ & $\begin{array}{l}\text { Carbomer, Fragrance, Isopropanol, Propylene Glycol, } \\
\text { Tocopheryl Acetate, Triethanolamine }\end{array}$ \\
\hline 44 & $\begin{array}{c}\text { Non- } \\
\text { gel }\end{array}$ & USA & $\begin{array}{l}\text { Isopropyl } \\
\text { Alcohol } 75 \% \\
\text { v/v }\end{array}$ & Glycerin, Hydrogen Peroxide, Purified Water USP \\
\hline 45 & Gel & USA & $\begin{array}{l}\text { Ethyl } \\
\text { Alcohol 70\% } \\
\text { v/v }\end{array}$ & $\begin{array}{l}\text { Water, Isopropyl Alcohol, Caprylyl Glycol, Glycerin, } \\
\text { Isopropyl Myristate, Tocopheryl Acetate }\end{array}$ \\
\hline
\end{tabular}




\begin{tabular}{|c|c|l|l|l|}
\hline $\mathbf{4 6}$ & Gel & USA & $\begin{array}{l}\text { Ethyl } \\
\text { Alcohol 70\% } \\
\text { v/v }\end{array}$ & $\begin{array}{l}\text { Purified Water, Isopropyl Alcohol, Propylene Glycol, } \\
\text { Fragrance, Aloe Barbadensis Leaf Juice (Decolorized), } \\
\text { Carbomer }\end{array}$ \\
\hline 47 & Gel & USA & $\begin{array}{l}\text { Ethyl Alcohol } \\
70 \% \text { v/v }\end{array}$ & Glycerin, Propylene Glycol, Purified water, Triethanolamine \\
\hline 48 & Gel & N/A & N/A & (Dispenser station at local store) \\
\hline
\end{tabular}

N/A indicates not applicable/unknown

*Based on a visual assessment by NIST of the product consistency in combination with the inactive ingredients.

Samples in bold text indicate hand sanitizers that were selected for detailed quantitative analysis. 
Appendix B: NMR estimated results for impurities below FDA interim limits.
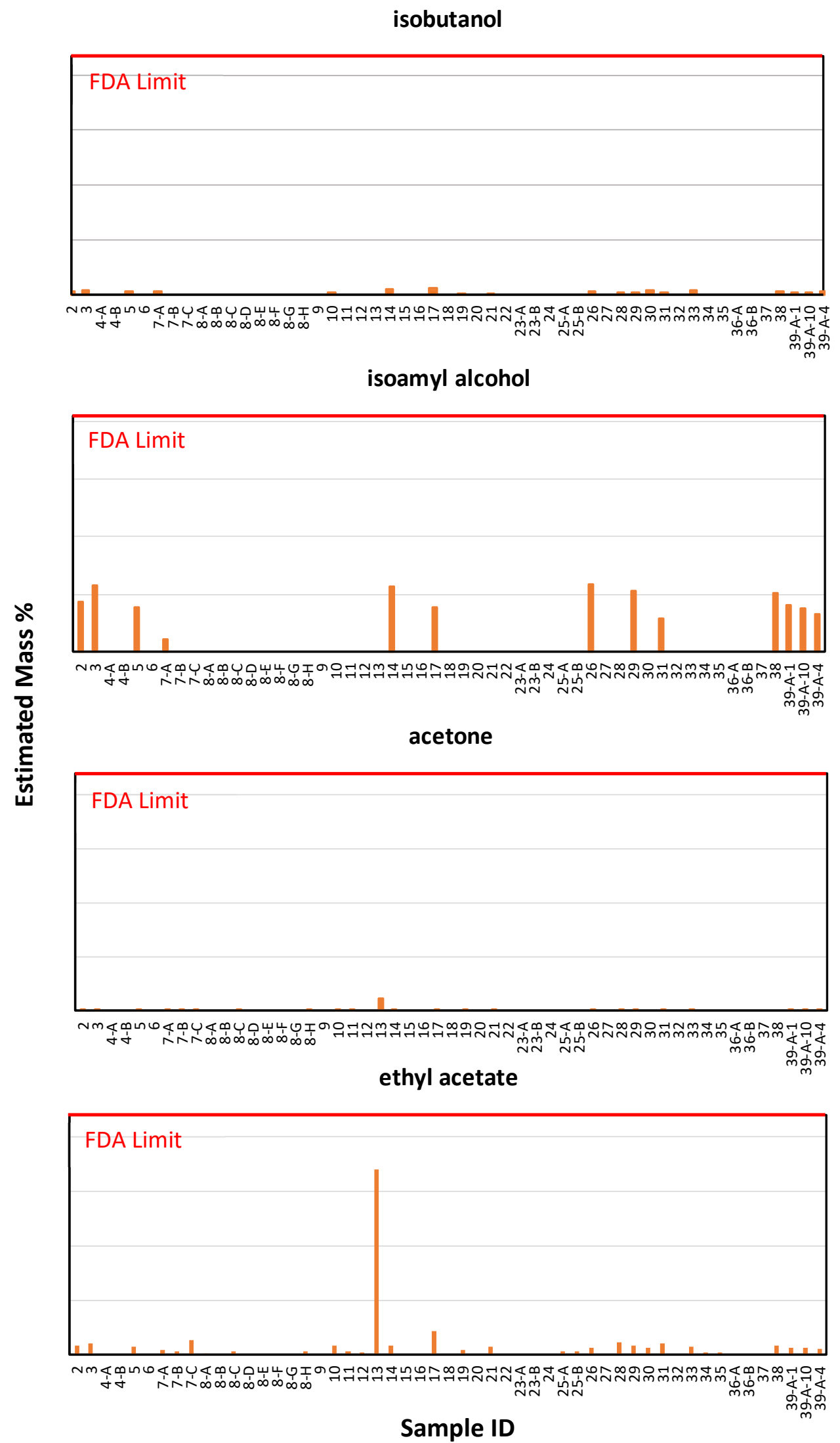
Appendix C. Plots of GC-FID results for impurities below FDA limits in some hand sanitizers.

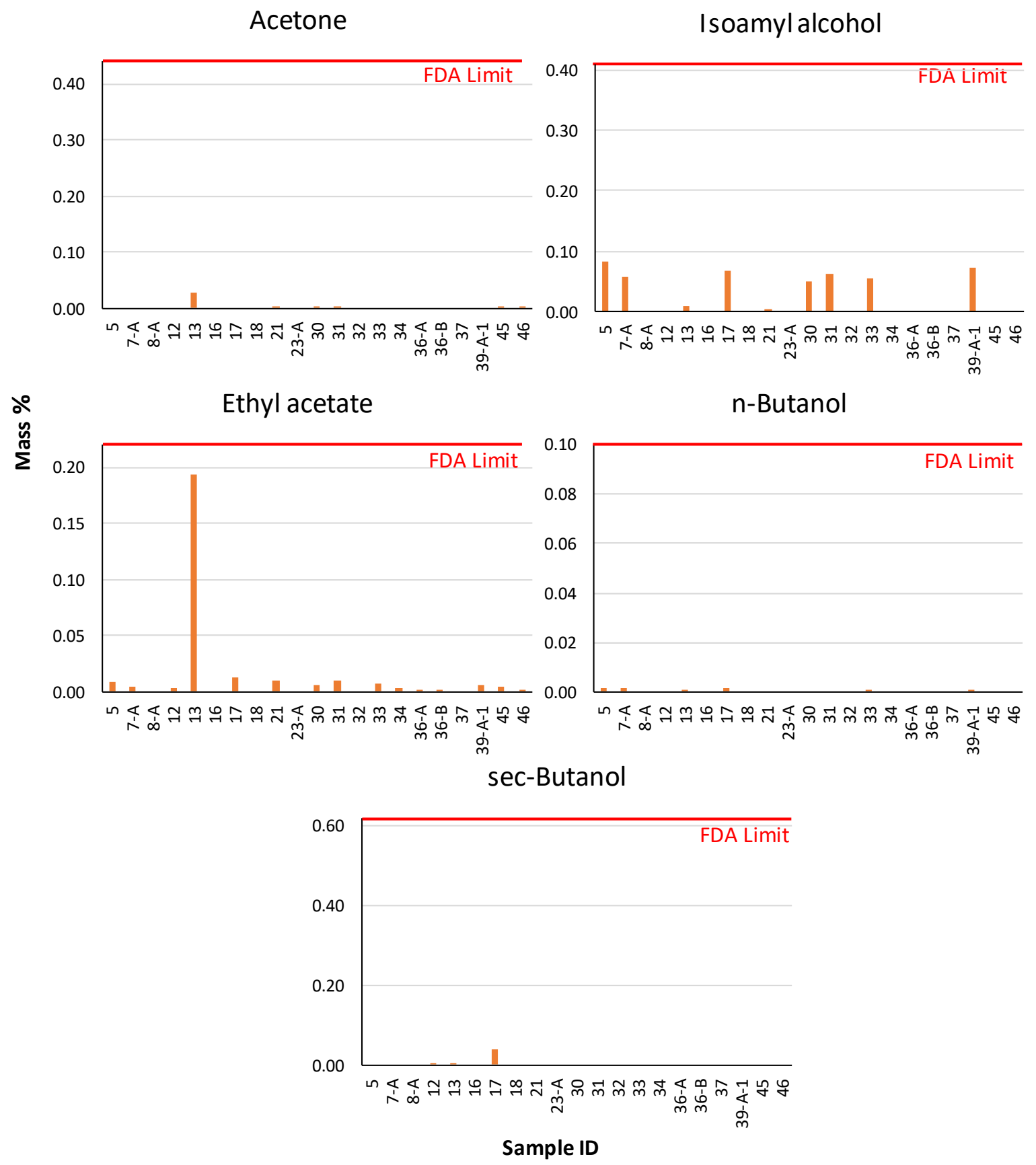


Appendix D. Density results for hand sanitizers $(\mathrm{g} / \mathrm{mL})$ obtained using a calibrated positive displacement pipette.

\begin{tabular}{|c|c|c|c|c|c|c|c|}
\hline $\begin{array}{l}\text { Sample } \\
\text { ID }\end{array}$ & $\begin{array}{l}\text { Temp } \\
\left({ }^{\circ} \mathrm{C}\right)\end{array}$ & $\begin{array}{c}\text { Replicate } \\
1 \\
\end{array}$ & $\begin{array}{c}\text { Replicate } \\
2 \\
\end{array}$ & $\begin{array}{c}\text { Replicate } \\
3 \\
\end{array}$ & Average & SD & $\% \mathrm{RSD}$ \\
\hline 1 & 21.6 & 0.848 & 0.848 & 0.847 & 0.848 & 0.001 & 0.11 \\
\hline 2 & 21.6 & 0.856 & 0.855 & 0.856 & 0.856 & 0.000 & 0.04 \\
\hline 3 & 21.6 & 0.851 & 0.852 & 0.852 & 0.852 & 0.001 & 0.12 \\
\hline $4-A$ & 21.6 & 0.833 & 0.835 & 0.833 & 0.834 & 0.001 & 0.09 \\
\hline $4-B$ & 21.6 & 0.845 & 0.845 & 0.844 & 0.845 & 0.001 & 0.09 \\
\hline 5 & 20.1 & 0.855 & 0.856 & 0.853 & 0.855 & 0.002 & 0.18 \\
\hline 6 & 21.6 & 0.955 & 0.959 & 0.958 & 0.957 & 0.002 & 0.18 \\
\hline $7-A$ & 21.6 & 0.870 & 0.868 & 0.868 & 0.869 & 0.001 & 0.12 \\
\hline 7-B & 20.5 & 0.861 & 0.859 & 0.859 & 0.860 & 0.001 & 0.11 \\
\hline 7-C & 21.6 & 0.868 & 0.869 & 0.869 & 0.869 & 0.001 & 0.07 \\
\hline 8-A & 21.6 & 0.926 & 0.925 & 0.924 & 0.925 & 0.001 & 0.09 \\
\hline $8-\mathrm{B}$ & 21.6 & 0.881 & 0.882 & 0.882 & 0.882 & 0.001 & 0.06 \\
\hline $8-\mathrm{C}$ & 21.6 & 0.853 & 0.858 & 0.857 & 0.856 & 0.003 & 0.31 \\
\hline 8-D & 20.1 & 0.892 & 0.896 & 0.893 & 0.894 & 0.002 & 0.26 \\
\hline 8-D & 21.6 & 0.899 & 0.899 & 0.900 & 0.899 & 0.000 & 0.03 \\
\hline $8-\mathrm{E}$ & 20.5 & 0.874 & 0.876 & 0.879 & 0.876 & 0.003 & 0.32 \\
\hline $8-\mathrm{F}$ & 21.6 & 0.833 & 0.834 & 0.833 & 0.833 & 0.001 & 0.07 \\
\hline $8-\mathrm{G}$ & 20.5 & 0.884 & 0.884 & 0.885 & 0.885 & 0.001 & 0.07 \\
\hline $8-\mathrm{H}$ & 20.5 & 0.895 & 0.883 & 0.884 & 0.887 & 0.007 & 0.76 \\
\hline 9 & 21.6 & 0.897 & 0.898 & 0.899 & 0.898 & 0.001 & 0.09 \\
\hline 10 & 21.6 & 0.862 & 0.863 & 0.862 & 0.863 & 0.001 & 0.06 \\
\hline 11 & 21.6 & 0.892 & 0.889 & 0.891 & 0.891 & 0.002 & 0.21 \\
\hline 12 & 20.1 & 0.903 & 0.899 & 0.901 & 0.901 & 0.002 & 0.19 \\
\hline 13 & 20.1 & 0.825 & 0.826 & 0.827 & 0.826 & 0.001 & 0.13 \\
\hline 14 & 21.6 & 0.882 & 0.881 & 0.882 & 0.882 & 0.000 & 0.04 \\
\hline 15 & 21.6 & 0.844 & 0.843 & 0.845 & 0.844 & 0.001 & 0.12 \\
\hline 16 & 21.6 & 0.860 & 0.857 & 0.859 & 0.858 & 0.001 & 0.17 \\
\hline 17 & 21.6 & 0.846 & 0.853 & 0.849 & 0.849 & 0.004 & 0.43 \\
\hline 18 & 21.6 & 0.878 & 0.878 & 0.879 & 0.878 & 0.001 & 0.09 \\
\hline 19 & 21.6 & 0.860 & 0.860 & 0.860 & 0.860 & 0.000 & 0.06 \\
\hline 20 & 21.6 & 0.855 & 0.856 & 0.857 & 0.856 & 0.001 & 0.10 \\
\hline 21 & 20.1 & 0.839 & 0.839 & 0.841 & 0.840 & 0.001 & 0.13 \\
\hline 22 & 20.5 & 0.887 & 0.892 & 0.892 & 0.890 & 0.003 & 0.33 \\
\hline $23-\mathrm{A}$ & 21.6 & 0.887 & 0.887 & 0.884 & 0.886 & 0.002 & 0.19 \\
\hline $23-\mathrm{B}$ & 20.5 & 0.886 & 0.886 & 0.885 & 0.886 & 0.001 & 0.07 \\
\hline 24 & 20.1 & 0.885 & 0.891 & 0.886 & 0.887 & 0.003 & 0.37 \\
\hline $25-\mathrm{A}$ & 20.5 & 0.873 & 0.870 & 0.872 & 0.872 & 0.002 & 0.20 \\
\hline $25-B$ & 21.6 & 0.846 & 0.847 & 0.846 & 0.846 & 0.001 & 0.06 \\
\hline 26 & 20.1 & 0.879 & 0.878 & 0.878 & 0.878 & 0.000 & 0.01 \\
\hline
\end{tabular}




\begin{tabular}{|c|c|c|c|c|c|c|c|}
\hline 27 & 20.5 & 0.881 & 0.883 & 0.879 & 0.881 & 0.002 & 0.21 \\
\hline 28 & 20.1 & 0.858 & 0.857 & 0.856 & 0.857 & 0.001 & 0.10 \\
\hline 29 & 20.5 & 0.884 & 0.886 & 0.886 & 0.886 & 0.001 & 0.12 \\
\hline 30 & 20.5 & 0.865 & 0.862 & 0.865 & 0.864 & 0.002 & 0.21 \\
\hline 31 & 20.1 & 0.882 & 0.881 & 0.885 & 0.883 & 0.002 & 0.22 \\
\hline 32 & 20.1 & 0.883 & 0.882 & 0.886 & 0.884 & 0.002 & 0.25 \\
\hline 33 & 20.1 & 0.858 & 0.857 & 0.852 & 0.855 & 0.003 & 0.39 \\
\hline 33 & 21.6 & 0.856 & 0.855 & 0.855 & 0.855 & 0.001 & 0.08 \\
\hline 34 & 20.1 & 0.875 & 0.872 & 0.871 & 0.873 & 0.002 & 0.23 \\
\hline $36-\mathrm{A}$ & 21.6 & 0.871 & 0.872 & 0.867 & 0.870 & 0.003 & 0.33 \\
\hline $36-\mathrm{B}$ & 21.6 & 0.874 & 0.875 & 0.874 & 0.874 & 0.001 & 0.06 \\
\hline 37 & 21.6 & 0.871 & 0.875 & 0.876 & 0.874 & 0.003 & 0.32 \\
\hline 38 & 21.6 & 0.842 & 0.841 & 0.841 & 0.841 & 0.000 & 0.05 \\
\hline 39-A-1 & 21.6 & 0.880 & 0.880 & 0.883 & 0.881 & 0.001 & 0.16 \\
\hline $39-A-2$ & 20.1 & 0.874 & 0.871 & 0.873 & 0.872 & 0.002 & 0.18 \\
\hline 40 & 21.6 & 0.842 & 0.849 & 0.844 & 0.845 & 0.004 & 0.43 \\
\hline 41 & 20.5 & 0.868 & 0.866 & 0.865 & 0.866 & 0.002 & 0.23 \\
\hline 42 & 21.6 & 0.851 & 0.853 & 0.852 & 0.852 & 0.001 & 0.09 \\
\hline 43 & 21.6 & 0.893 & 0.893 & 0.892 & 0.893 & 0.000 & 0.03 \\
\hline 44 & 21.6 & 0.898 & 0.900 & 0.900 & 0.899 & 0.001 & 0.13 \\
\hline 45 & 21.6 & 0.825 & 0.827 & 0.824 & 0.825 & 0.001 & 0.17 \\
\hline 46 & 21.6 & 0.887 & 0.885 & 0.883 & 0.885 & 0.002 & 0.22 \\
\hline
\end{tabular}

